\title{
Review of Sudanese medicinal plants screened for antifungal activity
}

\author{
Wail E. Abdalla 1, 2, Emad M. Abdallah 1,* \\ 1 Department of Laboratory Sciences, College of Sciences and Arts at Al-Rass, Qassim University, Saudi Arabia \\ 2 Medicinal and Aromatic Plants Research Institute, National Centre for Research, Sudan
}

\section{A R T I C L E I N F O}

Article history:

Received 20 June 2016

Received in revised form

11 September 2016

Accepted 12 September 2016

Keywords:

Antifungal activity

Antimicrobial

Sudanese plants

\begin{abstract}
A B S T R A C T
The present study aimed at reviewing Sudanese medicinal plants screened for antifungal activity. A total of 85 plant species belonging to 48 families (42 dicotyledonous and 6 monocotyledonous) were reported to have antifungal activity against some fungal species. The family Fabaceae was reported to be the most screened family, while Acacia nilotica from the same family and subfamily Mimosoideae was reported to be the most screened species. On the other hand, a total of 20 fungal species belonging to 10 genera and 6 families were reported to be used in studies for antifungal activities from Sudanese plants, with the two genera Aspergillus and Candida being the most tested genera. Four methods for testing antifungal activities of Sudanese plants were used, with the cup-plate diffusion method being the most applied method $(75.18 \%)$. It is hoped that this review will define the current situation of research for antifungal activities of Sudanese plants.

(C) 2016 The Authors. Published by IASE. This is an open access article under the CC BY-NC-ND license (http://creativecommons.org/licenses/by-nc-nd/4.0/).
\end{abstract}

\section{Introduction}

Since time immemorial, plants have been the integral part of the healthcare system, ancient fossil records showed that a medicinal plat called Hollyhock (Alcea rosea) have been used since 60000 years ago in present-day Iraq (Cowan, 1999). Medicinal plants are considered as the largest pharmaceutical stores on earth because they can produce an endless secondary phytochemical metabolites of bioactive properties which can be used effectively against various illnesses, and this was well understood and utilized by ancient civilizations (Abdallah, 2011). In the current era, although synthesized drugs are widely used in the modern medicine, the case differs in developing countries, where up to $80 \%$ of people there are still rely on traditional medicine for their health care needs, mostly plant drugs (Cunningham, 1997). Fungi considered as the second major part of biodiversity after insects, with up to 1.5 million species and only 400 species are known as animal and human pathogens (Razzaghi-Abyaneh and Rai, 2013).

\footnotetext{
* Corresponding Author.

Email Address: 140208@qu.edu.sa (E. Abdallah)

http://dx.doi.org/10.21833/ijaas.2016.08.014

2313-626X/C) 2016 The Authors. Published by IASE.

This is an open access article under the CC BY-NC-ND license

(http://creativecommons.org/licenses/by-nc-nd/4.0/)
}

The discovery of antifungal agents are much difficult than bacteria, since fungal cell wall mostly contains chitin and other polysaccharides which obstruct the antibiotics activity (Sasidharan et al., 2012), the similarity between the fungal cells and the mammalian cells could lead to toxicity of animal and human treated with these antifungal drugs (Scorzoni et al., 2016). The opportunistic fungi, particularly Aspergillus, Candida and Cryptococcus species, are life-threaten infections for the immunecompromised patients with AIDS, cancer or organtransplant (Yang et al., 2006). On the other side, the fungal plant diseases are increasing, representing more than $70 \%$ of the crop diseases and threatening so many agricultural and horticultural crops (Rodino et al., 2014).

Unlike the antibacterial drugs, most of the current antifungal drugs was initially derived from natural sources, for example, Amphotericin B was discovered from Streptomyces nodosus (Bacteria), Micafungin was derived from Coleophoma empetri (Fungus) and Caspofungin was obtained from Glarea lozoyensi (Fungus) (Scorzoni et al., 2016), but most of these antifungal drugs are not consistently effective due to the development of resistance in fungi and the serious side effects of these drugs, so plants that are rich in phytochemicals could represent an excellent source for antifungal drugs (Ahmad et al., 2010).

The geographical position of the Sudan in the center of different Arabian and African cultures helps in building a unique blend of traditional 
medical practices, relying mainly on herbal medicine (Khalid et al., 2012). Sudan contains within its borders a great diversity of climatic conditions and vegetation, correlating with these a wide variety of fungi and plant diseases (Omer, 2007). Besides, the critical health situation due to the deterioration of the health care services, has led the majority of the population to depend on the traditional medicinal plants as an alternative for the expensive modern drugs (Abdalla and Abdallah, 2016). However, Sudan possesses an immense wealth of bioactive medicinal plants that is still unexploited. The aim of this review is to highlight about Sudanese medicinal plants, which are screened for their antifungal activity against different fungi. This could be a precursor for a database that will define the current situation of antifungal research in the country and will help in planning the future.

\section{Materials and methods}

Data collection of medicinal plants screened for antifungal activity in Sudan was carried out during the period March 2016 to July 2016, by reviewing various published studies (Books, review papers, research papers, short communications, etc.) and unpublished MSc and $\mathrm{PhD}$ theses in Sudanese universities. The major scientific databases were searched, such as Google scholar, Science web of Knowledge, Science direct and Pubmed. Some criteria were put in consideration during gathering and tabulating the data of antifungal plants, such as: (i) Medicinal plant species which are collected only from Sudan;

(ii) With reported antifungal activity;

(iii) Which are investigated in vitro and the method used was mentioned;

(iv) Full information about the target plant were explained, such as the plant family, the scientific and vernacular names (which are usually used by the local people instead of the scientific names), the tested part of the plant and the solvent used in the extraction of the active constituents;

(v) Based on literature, the fungi tested against Sudanese plants were Aspergillus niger, A. flavus, A. fumigatus, Candida albicans, Fusarium oxysporum, F. culmorum, F. graminearum, Penicillium italicum, P. digitatum, Phialophora richardsiae, Tricophyton verrucosum, T. rubrum, T. concentricum, $T$. tonsurans , T. mentagrophytes, Microsporum canis, M. audouinii, Saccharomyces cerevisiae, Madurella mycetomatis, Epidermophyton floccosum.

(vi) For facilitation, the antifungal efficacy of the target plants was expressed as $(+)$ which means presence of antifungal activity, or as $(-)$ which means absence of antifungal activity, based on the researcher statement.

\section{Results and discussion}

As shown in Table 1, detailed information about the plants and their antifungal properties are presented. It was revealed that, up to 85 plant species belonging to 48 families (42 dicotyledonous and 6 monocotyledonous) were reported to have antifungal activity against some fungal species.

It was shown in Fig. 1 that Fabaceae, being represented by 10 species, was the most family tested for antifungal activity, followed by Asteraceae (5 species), Brassicaceiae, Capparaceae and Malvaceae (4 species), then Burseraceae, Combretaceae and Euphorbiaceae (3 species). The rest of families were only represented by either two or one species. The family Fabaceae is considered a large family, divided into three subfamilies represented in this study by: Caesalpinioideae (1 species), Faboideae (6 species) and Mimosoideae (3 species), where subfamily Faboideae still represent the most tested taxum. However, the species Acacia nilotica (subfamily Mimosoideae) received the greatest attention of Sudanese researchers for antifungal activity, where 4 parts of this species were tested using different solvents and methods of extraction with a total of 34 tests of this single plant species against different fungal species (Table 1). This attention could be attributed to the wide use of this species in Sudanese traditional medicine (Brown and Massey, 1929; El Ghazali et al., 1987; El Ghazali et al., 1994; El Ghazali et al., 1997) as well as rural tanning industry (Musa and Gasmelseed, 2014).

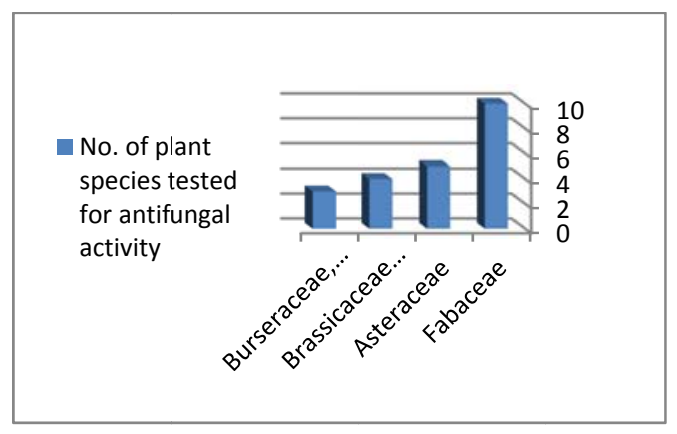

Fig. 1: Number of plant species tested for antifungal activity per family

The main methods used for investigating the Sudanese plants for their antifungal activity in this review, were the cup-plate diffusion method $(75.18 \%)$, followed by the mean radial growth of mycelium or the dry/wet weight of mycelium $(12.7 \%)$, the least method used was the disc diffusion method (8\%) and the minimum inhibitory concentration method (8\%). It was reported that, the main methods for in vitro antifungal screening are diffusion method, minimum inhibitory concentration (MIC), minimum fungicidal concentration (MFC) and $\mathrm{IC}_{50}$ value on hyphal growth inhibition as well as many other in vivo and in situ methods (Sasidharan et al., 2012). The majority of these published studies are not funded, reflecting the need to pay more attention and support to the scientific efforts in this developing country, which is rich in medicinal plants.

In addition, systematic and integrated studies on Sudanese medicinal plants in order to discover new antimicrobial drugs are absent; the majority of the studies are at the screening level on crude extracts. 
Table 1: Sudanese medicinal plants screened for antifungal activity

\begin{tabular}{|c|c|c|c|c|c|c|c|c|c|c|c|c|c|c|c|c|c|c|c|c|c|c|c|c|c|c|}
\hline Plant Family & $\begin{array}{c}\text { Plant Scientific } \\
\text { Name }\end{array}$ & $\begin{array}{c}\text { Plant } \\
\text { Vernacular } \\
\text { Name } \\
\end{array}$ & $\begin{array}{l}\text { Plant } \\
\text { Part }\end{array}$ & $\begin{array}{l}\text { Method } \\
\text { used }\end{array}$ & $\begin{array}{c}\text { Solvent } \\
\text { used }\end{array}$ & : & $\stackrel{\vec{R}}{\overrightarrow{2}}$ & $\stackrel{P}{\vec{s}}$ & $\hat{\imath}$ & $\vec{x}$ & $\stackrel{3}{\vdots}$ & $\begin{array}{l}3 \\
0 \\
0 \\
0\end{array}$ & $\begin{array}{l}\text { Acti } \\
\nabla \\
\tilde{\sigma}\end{array}$ & tivit & 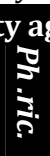 & 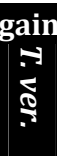 & $\mid \begin{array}{l}n \\
\vdots \\
3 \\
-3\end{array}$ & $\begin{array}{l}\text { ung } \\
0 \\
3 \\
3 \\
0\end{array}$ & $\mid \vec{i}$ & $\mid \begin{array}{r}-1 \\
\hat{S}\end{array}$ & $\mid \begin{array}{c}3 \\
\vdots \\
\vdots \\
5\end{array}$ & $\begin{array}{l}3 \\
\vdots \\
\vdots \\
\vdots\end{array}$ & $\ddot{9}$ & $\frac{0}{0}$ & 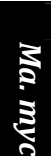 & References \\
\hline \multirow{11}{*}{ Acanthaceae } & \multirow{3}{*}{$\begin{array}{c}\text { Hygrophila } \\
\text { auriculata } \\
\text { (Schumach.) Heine } \\
\text { (Asteracantha } \\
\text { auriculata Nees) }\end{array}$} & \multirow{3}{*}{$\begin{array}{c}\text { Abu } \\
\text { Shwaika }\end{array}$} & \multirow[b]{3}{*}{ W.P. } & \multirow[b]{3}{*}{ M1 } & $\mathrm{CHCl}_{3}$ & $\mathrm{~N}$ & $\mathrm{~N}$ & $\mathrm{~N}$ & - & $\mathrm{N}$ & $\mathrm{N}$ & $\mathrm{N}$ & $\mathrm{N}$ & $\mathrm{N}$ & $\mathrm{N}$ & $\mathrm{N}$ & $\mathrm{N}$ & $\mathrm{N}$ & $\mathrm{N}$ & $\mathrm{N}$ & $\mathrm{N}$ & $\mathrm{N}$ & - & $\mathrm{N}$ & $\mathrm{N}$ & \multirow{3}{*}{$\begin{array}{l}\text { El-Egami et } \\
\text { al. } 2005\end{array}$} \\
\hline & & & & & $\mathrm{MeOH}$ & $\mathrm{N}$ & $\mathrm{N}$ & $\mathrm{N}$ & - & $\mathrm{N}$ & $\mathrm{N}$ & $\mathrm{N}$ & $\mathrm{N}$ & $\mathrm{N}$ & $\mathrm{N}$ & $\mathrm{N}$ & $\mathrm{N}$ & $\mathrm{N}$ & $\mathrm{N}$ & $\mathrm{N}$ & $\mathrm{N}$ & $\mathrm{N}$ & - & $\mathrm{N}$ & $\mathrm{N}$ & \\
\hline & & & & & $\mathrm{H}_{2} \mathrm{O}$ & $\mathrm{N}$ & $\mathrm{N}$ & $\mathrm{N}$ & - & $\mathrm{N}$ & $\mathrm{N}$ & $\mathrm{N}$ & $\mathrm{N}$ & $\mathrm{N}$ & $\mathrm{N}$ & $\mathrm{N}$ & $\mathrm{N}$ & $\mathrm{N}$ & $\mathrm{N}$ & $\mathrm{N}$ & $\mathrm{N}$ & $\mathrm{N}$ & - & $\mathrm{N}$ & $\mathrm{N}$ & \\
\hline & \multirow{8}{*}{$\begin{array}{l}\text { Monechma ciliatum } \\
\text { (Jacq.) Milne-Redh. }\end{array}$} & \multirow{8}{*}{$\begin{array}{c}\text { Black } \\
\text { Mahlab }\end{array}$} & \multirow{3}{*}{ St. } & \multirow{3}{*}{ M1 } & $\mathrm{MeOH}$ & - & $\mathrm{N}$ & $\mathrm{N}$ & + & $\mathrm{N}$ & $\mathrm{N}$ & $\mathrm{N}$ & $\mathrm{N}$ & $\mathrm{N}$ & $\mathrm{N}$ & $\mathrm{N}$ & $\mathrm{N}$ & $\mathrm{N}$ & $\mathrm{N}$ & $\mathrm{N}$ & $\mathrm{N}$ & $\mathrm{N}$ & $\mathrm{N}$ & $\mathrm{N}$ & $\mathrm{N}$ & \multirow{6}{*}{$\begin{array}{l}\text { Abuelgasim } \\
\text { et al } 2015\end{array}$} \\
\hline & & & & & $\mathrm{CHCl}_{3}$ & - & $\mathrm{N}$ & $\mathrm{N}$ & + & $\mathrm{N}$ & $\mathrm{N}$ & $\mathrm{N}$ & $\mathrm{N}$ & $\mathrm{N}$ & $\mathrm{N}$ & $\mathrm{N}$ & $\mathrm{N}$ & $\mathrm{N}$ & $\mathrm{N}$ & $\mathrm{N}$ & $\mathrm{N}$ & $\mathrm{N}$ & $\mathrm{N}$ & $\mathrm{N}$ & $\mathrm{N}$ & \\
\hline & & & & & $\mathrm{H}_{2} \mathrm{O}$ & - & $\mathrm{N}$ & $\mathrm{N}$ & - & $\mathrm{N}$ & $\mathrm{N}$ & $\mathrm{N}$ & $\mathrm{N}$ & $\mathrm{N}$ & $\mathrm{N}$ & $\mathrm{N}$ & $\mathrm{N}$ & $\mathrm{N}$ & $\mathrm{N}$ & $\mathrm{N}$ & $\mathrm{N}$ & $\mathrm{N}$ & $\mathrm{N}$ & $\mathrm{N}$ & $\mathrm{N}$ & \\
\hline & & & \multirow{3}{*}{ Le. } & \multirow{3}{*}{ M1 } & $\mathrm{MeOH}$ & - & $\mathrm{N}$ & $\mathrm{N}$ & - & $\mathrm{N}$ & $\mathrm{N}$ & $\mathrm{N}$ & $\mathrm{N}$ & $\mathrm{N}$ & $\mathrm{N}$ & $\mathrm{N}$ & $\mathrm{N}$ & $\mathrm{N}$ & $\mathrm{N}$ & $\mathrm{N}$ & $\mathrm{N}$ & $\mathrm{N}$ & $\mathrm{N}$ & $\mathrm{N}$ & $\mathrm{N}$ & \\
\hline & & & & & $\mathrm{CHCl}_{3}$ & \begin{tabular}{|l|}
- \\
\end{tabular} & $\mathrm{N}$ & $\mathrm{N}$ & + & $\mathrm{N}$ & \begin{tabular}{|l|}
$\mathrm{N}$ \\
\end{tabular} & $\mathrm{N}$ & $\mathrm{N}$ & $\mathrm{N}$ & $\mathrm{N}$ & $\mathrm{N}$ & $\mathrm{N}$ & $\mathrm{N}$ & $\mathrm{N}$ & $\mathrm{N}$ & $\mathrm{N}$ & $\mathrm{N}$ & $\mathrm{N}$ & $\mathrm{N}$ & $\mathrm{N}$ & \\
\hline & & & & & $\mathrm{H}_{2} \mathrm{O}$ & - & $\mathrm{N}$ & $\mathrm{N}$ & - & $\mathrm{N}$ & \begin{tabular}{|l|}
$\mathrm{N}$ \\
\end{tabular} & $\mathrm{N}$ & $\mathrm{N}$ & $\mathrm{N}$ & $\mathrm{N}$ & $\mathrm{N}$ & $\mathrm{N}$ & $\mathrm{N}$ & $\mathrm{N}$ & $\mathrm{N}$ & $\mathrm{N}$ & $\mathrm{N}$ & $\mathrm{N}$ & $\mathrm{N}$ & $\mathrm{N}$ & \\
\hline & & & \multirow[b]{2}{*}{ Se. } & \multirow{2}{*}{ M1 } & $\mathrm{H}_{2} \mathrm{O}$ & + & - & $\mathrm{N}$ & + & $\mathrm{N}$ & $\mathrm{N}$ & $\mathrm{N}$ & $\mathrm{N}$ & $\mathrm{N}$ & $\mathrm{N}$ & $\mathrm{N}$ & $\mathrm{N}$ & $\mathrm{N}$ & $\mathrm{N}$ & $\mathrm{N}$ & $\mathrm{N}$ & $\mathrm{N}$ & $\mathrm{N}$ & $\mathrm{N}$ & $\mathrm{N}$ & \multirow{2}{*}{ Oshi 2013} \\
\hline & & & & & $\mathrm{MeOH}$ & + & - & $\mathrm{N}$ & - & \begin{tabular}{|l|}
$\mathrm{N}$ \\
\end{tabular} & \begin{tabular}{|l|}
$\mathrm{N}$ \\
\end{tabular} & \begin{tabular}{|l|}
$\mathrm{N}$ \\
\end{tabular} & \begin{tabular}{|l|}
$\mathrm{N}$ \\
\end{tabular} & $\mathrm{N}$ & $\mathrm{N}$ & \begin{tabular}{|l|}
$\mathrm{N}$ \\
\end{tabular} & $\mathrm{N}$ & $\mathrm{N}$ & $\mathrm{N}$ & \begin{tabular}{|l|}
$\mathrm{N}$ \\
\end{tabular} & $\mathrm{N}$ & $\mathrm{N}$ & \begin{tabular}{|l|}
$\mathrm{N}$ \\
\end{tabular} & $\mathrm{N}$ & $\mathrm{N}$ & \\
\hline \multirow{3}{*}{ Alismataceae } & \multirow{3}{*}{$\begin{array}{l}\text { Limnophyton } \\
\text { obtusifolium (L.) } \\
\text { Miq. }\end{array}$} & \multirow{3}{*}{-} & \multirow{3}{*}{ W.P. } & \multirow{3}{*}{ M1 } & $\mathrm{CHCl}_{3}$ & $\mathrm{~N}$ & $\mathrm{~N}$ & $\mathrm{~N}$ & - & $\mathrm{N}$ & $\mathrm{N}$ & $\mathrm{N}$ & $\mathrm{N}$ & $\mathrm{N}$ & $\mathrm{N}$ & $\mathrm{N}$ & $\mathrm{N}$ & $\mathrm{N}$ & $\mathrm{N}$ & $\mathrm{N}$ & $\mathrm{N}$ & $\mathrm{N}$ & + & $\mathrm{N}$ & $\mathrm{N}$ & \\
\hline & & & & & $\mathrm{MeOH}$ & $\mathrm{N}$ & $\mathrm{N}$ & $\mathrm{N}$ & + & \begin{tabular}{|l|}
$\mathrm{N}$ \\
\end{tabular} & \begin{tabular}{|l|}
$\mathrm{N}$ \\
\end{tabular} & $\mathrm{N}$ & $\mathrm{N}$ & $\mathrm{N}$ & $\mathrm{N}$ & $\mathrm{N}$ & $\mathrm{N}$ & $\mathrm{N}$ & $\mathrm{N}$ & $\mathrm{N}$ & $\mathrm{N}$ & $\mathrm{N}$ & + & $\mathrm{N}$ & $\mathrm{N}$ & El-Egami et \\
\hline & & & & & $\mathrm{H}_{2} \mathrm{O}$ & $\mathrm{N}$ & $\mathrm{N}$ & $\mathrm{N}$ & - & $\mathrm{N}$ & $\mathrm{N}$ & $\mathrm{N}$ & $\mathrm{N}$ & $\mathrm{N}$ & $\mathrm{N}$ & $\mathrm{N}$ & $\mathrm{N}$ & $\mathrm{N}$ & $\mathrm{N}$ & $\mathrm{N}$ & $\mathrm{N}$ & $\mathrm{N}$ & - & $\mathrm{N}$ & $\mathrm{N}$ & \\
\hline Amaranthacea & Achyranthes aspera & Kashm & $\begin{array}{l}\text { Se, } \\
\text { St, } \\
\text { Fr }\end{array}$ & M1 & $\begin{array}{l}\text { Pet. } \\
\text { ether }\end{array}$ & + & $\mathrm{N}$ & $\mathrm{N}$ & + & $\mathrm{N}$ & $\mathrm{N}$ & $\mathrm{N}$ & $\mathrm{N}$ & $\mathrm{N}$ & $\mathrm{N}$ & $\mathrm{N}$ & $\mathrm{N}$ & $\mathrm{N}$ & $\mathrm{N}$ & $\mathrm{N}$ & $\mathrm{N}$ & $\mathrm{N}$ & $\mathrm{N}$ & $\mathrm{N}$ & $\mathrm{N}$ & Burham \\
\hline & & Alnasseba & 11 & M1 & EtOAc & + & $\mathrm{N}$ & $\mathrm{N}$ & + & \begin{tabular}{|l|}
$\mathrm{N}$ \\
\end{tabular} & \begin{tabular}{|l|}
$\mathrm{N}$ \\
\end{tabular} & $\mathrm{N}$ & $\mathrm{N}$ & $\mathrm{N}$ & $\mathrm{N}$ & $\mathrm{N}$ & $\mathrm{N}$ & $\mathrm{N}$ & $\mathrm{N}$ & $\mathrm{N}$ & $\mathrm{N}$ & $\mathrm{N}$ & \begin{tabular}{|l|}
$\mathrm{N}$ \\
\end{tabular} & $\mathrm{N}$ & $\mathrm{N}$ & 2013 \\
\hline & & & $\mathrm{Fr}$ & M1 & $\mathrm{MeOH}$ & - & $\mathrm{N}$ & $\mathrm{N}$ & - & $\mathrm{N}$ & \begin{tabular}{|l|}
$\mathrm{N}$ \\
\end{tabular} & $\mathrm{N}$ & $\mathrm{N}$ & $\mathrm{N}$ & $\mathrm{N}$ & $\mathrm{N}$ & $\mathrm{N}$ & $\mathrm{N}$ & $\mathrm{N}$ & $\mathrm{N}$ & $\mathrm{N}$ & $\mathrm{N}$ & $\mathrm{N}$ & $\mathrm{N}$ & $\mathrm{N}$ & \\
\hline & Allium cepa L. & Bessel & Pulb & M3 & $\mathrm{H}_{2} \mathrm{O}$ & + & $\mathrm{N}$ & $\mathrm{N}$ & $\mathrm{N}$ & $\mathrm{N}$ & $\mathrm{N}$ & $\mathrm{N}$ & $\mathrm{N}$ & + & $\mathrm{N}$ & $\mathrm{N}$ & $\mathrm{N}$ & $\mathrm{N}$ & $\mathrm{N}$ & $\mathrm{N}$ & $\mathrm{N}$ & $\mathrm{N}$ & $\mathrm{N}$ & $\mathrm{N}$ & $\mathrm{N}$ & Abdel- \\
\hline Amaryllidaceae & Allium sativum $\mathrm{L}$. & Toom & Pulb & M3 & $\mathrm{H}_{2} \mathrm{O}$ & + & $\mathrm{N}$ & $\mathrm{N}$ & $\mathrm{N}$ & $\mathrm{N}$ & $\mathrm{N}$ & $\mathrm{N}$ & $\mathrm{N}$ & + & $\mathrm{N}$ & $\mathrm{N}$ & $\mathrm{N}$ & $\mathrm{N}$ & $\mathrm{N}$ & $\mathrm{N}$ & $\mathrm{N}$ & $\mathrm{N}$ & $\mathrm{N}$ & $\mathrm{N}$ & $\mathrm{N}$ & $\begin{array}{l}\text { Rahim et al. } \\
\text { 2012b }\end{array}$ \\
\hline $\begin{array}{c}\text { Apiaceae/ } \\
\text { Umbelliferae }\end{array}$ & $\begin{array}{l}\text { Cuminum cyminum } \\
\text { L. }\end{array}$ & Shamar & Fr. & M1 & Ess. oil & + & $\mathrm{N}$ & $\mathrm{N}$ & + & $\mathrm{N}$ & $\mathrm{N}$ & $\mathrm{N}$ & $\mathrm{N}$ & $\mathrm{N}$ & $\mathrm{N}$ & $\mathrm{N}$ & $\mathrm{N}$ & $\mathrm{N}$ & $\mathrm{N}$ & $\mathrm{N}$ & $\mathrm{N}$ & $\mathrm{N}$ & $\mathrm{N}$ & $\mathrm{N}$ & $\mathrm{N}$ & $\begin{array}{l}\text { Abushama } \\
\text { et al. } 2013\end{array}$ \\
\hline & & & & & $\mathrm{CHCl}_{3}$ & $\mathrm{~N}$ & $\mathrm{~N}$ & $\mathrm{~N}$ & + & $\mathrm{N}$ & $\mathrm{N}$ & $\mathrm{N}$ & $\mathrm{N}$ & $\mathrm{N}$ & $\mathrm{N}$ & $\mathrm{N}$ & $\mathrm{N}$ & $\mathrm{N}$ & $\mathrm{N}$ & $\mathrm{N}$ & $\mathrm{N}$ & $\mathrm{N}$ & + & $\mathrm{N}$ & $\mathrm{N}$ & El-Egami et \\
\hline Arecaceae/ & Hyphaene thebaica & Dome & Fr. & M1 & $\mathrm{MeOH}$ & $\mathrm{N}$ & $\mathrm{N}$ & $\mathrm{N}$ & + & $\mathrm{N}$ & $\mathrm{N}$ & $\mathrm{N}$ & $\mathrm{N}$ & $\mathrm{N}$ & $\mathrm{N}$ & $\mathrm{N}$ & $\mathrm{N}$ & $\mathrm{N}$ & $\mathrm{N}$ & $\mathrm{N}$ & $\mathrm{N}$ & $\mathrm{N}$ & + & $\mathrm{N}$ & $\mathrm{N}$ & al. 2006 \\
\hline & & & & & $\mathrm{H}_{2} \mathrm{O}$ & $\mathrm{N}$ & $\mathrm{N}$ & $\mathrm{N}$ & + & $\mathrm{N}$ & $\mathrm{N}$ & $\mathrm{N}$ & $\mathrm{N}$ & $\mathrm{N}$ & $\mathrm{N}$ & $\mathrm{N}$ & $\mathrm{N}$ & $\mathrm{N}$ & $\mathrm{N}$ & $\mathrm{N}$ & $\mathrm{N}$ & $\mathrm{N}$ & + & $\mathrm{N}$ & $\mathrm{N}$ & \\
\hline & Leptadenia & & Le. & & & $\mathrm{N}$ & $\mathrm{N}$ & $\mathrm{N}$ & $\mathrm{N}$ & $\mathrm{N}$ & $\mathrm{N}$ & $\mathrm{N}$ & $\mathrm{N}$ & $\mathrm{N}$ & $\mathrm{N}$ & $\mathrm{N}$ & $\mathrm{N}$ & $\mathrm{N}$ & $\mathrm{N}$ & $\mathrm{N}$ & + & $\mathrm{N}$ & $\mathrm{N}$ & $\mathrm{N}$ & $\mathrm{N}$ & \\
\hline & $\begin{array}{c}\text { arborea (Forssk.) } \\
\text { Schweinf. }\end{array}$ & Lewais & St. & M1 & EtOH & $\mathrm{N}$ & $\mathrm{N}$ & $\mathrm{N}$ & $\mathrm{N}$ & $\mathrm{N}$ & $\mathrm{N}$ & $\mathrm{N}$ & $\mathrm{N}$ & $\mathrm{N}$ & $\mathrm{N}$ & $\mathrm{N}$ & $\mathrm{N}$ & $\mathrm{N}$ & $\mathrm{N}$ & $\mathrm{N}$ & + & $\mathrm{N}$ & $\mathrm{N}$ & $\mathrm{N}$ & $\mathrm{N}$ & $\begin{array}{l}\text { Elshikh et } \\
\text { al. } 2016\end{array}$ \\
\hline & & & & & $\mathrm{CHCl}_{3}$ & - & $\mathrm{N}$ & $\mathrm{N}$ & + & $\mathrm{N}$ & $\mathrm{N}$ & $\mathrm{N}$ & $\mathrm{N}$ & $\mathrm{N}$ & $\mathrm{N}$ & $\mathrm{N}$ & $\mathrm{N}$ & $\mathrm{N}$ & $\mathrm{N}$ & $\mathrm{N}$ & $\mathrm{N}$ & $\mathrm{N}$ & $\mathrm{N}$ & $\mathrm{N}$ & $\mathrm{N}$ & \\
\hline Asclepiadaceae & & & & M1 & $\mathrm{MeOH}$ & - & $\mathrm{N}$ & $\mathrm{N}$ & - & \begin{tabular}{|l|l|}
14 \\
\end{tabular} & \begin{tabular}{|l|} 
\\
$\mathrm{N}$
\end{tabular} & $\mathrm{N}$ & $\mathrm{N}$ & $\mathrm{N}$ & $\mathrm{N}$ & $\mathrm{N}$ & $\mathrm{N}$ & $\mathrm{N}$ & $\mathrm{N}$ & $\mathrm{N}$ & $\mathrm{N}$ & $\mathrm{N}$ & $\mathrm{N}$ & $\mathrm{N}$ & $\mathrm{N}$ & Abdalla \\
\hline & $\begin{array}{l}\text { Solenostemma } \\
\text { argel (Del) Havne }\end{array}$ & Hargel & Le. & & $\mathrm{H}_{2} \mathrm{O}$ & - & $\mathrm{N}$ & $\mathrm{N}$ & - & $\mathrm{N}$ & $\mathrm{N}$ & $\mathrm{N}$ & $\mathrm{N}$ & $\mathrm{N}$ & $\mathrm{N}$ & $\mathrm{N}$ & $\mathrm{N}$ & $\mathrm{N}$ & $\mathrm{N}$ & $\mathrm{N}$ & $\mathrm{N}$ & $\mathrm{N}$ & $\mathrm{N}$ & $\mathrm{N}$ & $\mathrm{N}$ & \\
\hline & & & & M3 & $\mathrm{H}_{2} \mathrm{O}$ & + & $\mathrm{N}$ & $\mathrm{N}$ & + & $\mathrm{N}$ & $\mathrm{N}$ & $\mathrm{N}$ & $\mathrm{N}$ & $\mathrm{N}$ & $\mathrm{N}$ & $\mathrm{N}$ & $\mathrm{N}$ & $\mathrm{N}$ & $\mathrm{N}$ & $\mathrm{N}$ & $\mathrm{N}$ & $\mathrm{N}$ & $\mathrm{N}$ & $\mathrm{N}$ & $\mathrm{N}$ & $\begin{array}{l}\text { Suleiman et } \\
\text { al. } 2009\end{array}$ \\
\hline
\end{tabular}




\begin{tabular}{|c|c|c|c|c|c|c|c|c|c|c|c|c|c|c|c|c|c|c|c|c|c|c|c|c|c|c|}
\hline Plant Family & $\begin{array}{l}\text { Plant Scientific } \\
\text { Name }\end{array}$ & $\begin{array}{c}\text { Plant } \\
\text { Vernacular } \\
\text { Name }\end{array}$ & $\begin{array}{l}\text { Plant } \\
\text { Part }\end{array}$ & $\begin{array}{c}\text { Method } \\
\text { used }\end{array}$ & $\begin{array}{c}\text { Solvent } \\
\text { used }\end{array}$ & : & $\stackrel{\vec{s}}{\vec{S}}$ & 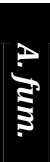 & $\stackrel{?}{\hat{\sigma}}$ & 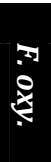 & $\stackrel{-1}{\vdots}$ & $\stackrel{x}{0}$ & \begin{tabular}{l} 
Act \\
$: 0$ \\
\multirow{5}{5}{}
\end{tabular} & ivit & 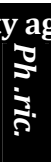 & 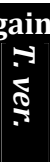 & $\begin{array}{l}\text { st } \mathbf{f} \\
-1 \\
\vdots \\
\vdots \\
\vdots\end{array}$ & $\begin{array}{l}\text { ung } \\
0 \\
3 \\
0 \\
0\end{array}$ & $\mid \vec{i}$ & 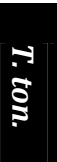 & 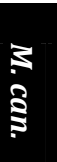 & 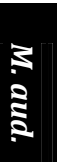 & ș & $\frac{2}{2}$ & 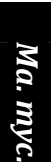 & References \\
\hline $\begin{array}{l}\text { Asteraceae/ } \\
\text { Compositae }\end{array}$ & $\begin{array}{l}\text { Acanthospermum } \\
\text { hispidum DC. }\end{array}$ & $\begin{array}{l}\text { Hurab } \\
\text { Hawsa }\end{array}$ & A.P. & M1 & $\begin{array}{l}\text { Pet. } \\
\text { Ether }\end{array}$ & + & $\mathrm{N}$ & $\mathrm{N}$ & + & $\mathrm{N}$ & $\mathrm{N}$ & $\mathrm{N}$ & $\mathrm{N}$ & $\mathrm{N}$ & $\mathrm{N}$ & $\mathrm{N}$ & $\mathrm{N}$ & $\mathrm{N}$ & $\mathrm{N}$ & $\mathrm{N}$ & $\mathrm{N}$ & $\mathrm{N}$ & $\mathrm{N}$ & $\mathrm{N}$ & $\mathrm{N}$ & $\begin{array}{c}\text { Burham } \\
2013\end{array}$ \\
\hline \multirow{14}{*}{$\begin{array}{l}\text { Asteraceae/ } \\
\text { Compositae }\end{array}$} & \multirow{2}{*}{$\begin{array}{l}\text { Acanthospermum } \\
\text { hispidum DC. }\end{array}$} & \multirow{2}{*}{$\begin{array}{l}\text { Hurab } \\
\text { Hawsa }\end{array}$} & \multirow{2}{*}{ A.P. } & \multirow{2}{*}{ M1 } & EtOAc & - & $\mathrm{N}$ & $\mathrm{N}$ & - & $\mathrm{N}$ & $\mathrm{N}$ & $\mathrm{N}$ & $\mathrm{N}$ & $\mathrm{N}$ & $\mathrm{N}$ & $\mathrm{N}$ & $\mathrm{N}$ & $\mathrm{N}$ & $\mathrm{N}$ & $\mathrm{N}$ & $\mathrm{N}$ & $\mathrm{N}$ & $\mathrm{N}$ & $\mathrm{N}$ & $\mathrm{N}$ & \multirow{5}{*}{$\begin{array}{c}\text { Burham } \\
2013\end{array}$} \\
\hline & & & & & $\mathrm{MeOH}$ & + & $\mathrm{N}$ & $\mathrm{N}$ & + & $\mathrm{N}$ & $\mathrm{N}$ & $\mathrm{N}$ & $\mathrm{N}$ & $\mathrm{N}$ & $\mathrm{N}$ & $\mathrm{N}$ & $\mathrm{N}$ & $\mathrm{N}$ & $\mathrm{N}$ & $\mathrm{N}$ & $\mathrm{N}$ & $\mathrm{N}$ & $\mathrm{N}$ & $\mathrm{N}$ & $\mathrm{N}$ & \\
\hline & \multirow{3}{*}{$\begin{array}{c}\text { Ambrosia maritima } \\
\text { L. }\end{array}$} & \multirow{3}{*}{ Damsisa } & \multirow{3}{*}{ W.P. } & \multirow{3}{*}{ M1 } & $\begin{array}{c}\text { Pet. } \\
\text { Ether }\end{array}$ & - & $\mathrm{N}$ & $\mathrm{N}$ & + & $\mathrm{N}$ & $\mathrm{N}$ & $\mathrm{N}$ & $\mathrm{N}$ & $\mathrm{N}$ & $\mathrm{N}$ & $\mathrm{N}$ & $\mathrm{N}$ & $\mathrm{N}$ & $\mathrm{N}$ & $\mathrm{N}$ & $\mathrm{N}$ & $\mathrm{N}$ & $\mathrm{N}$ & $\mathrm{N}$ & $\mathrm{N}$ & \\
\hline & & & & & EtOAc & - & $\mathrm{N}$ & $\mathrm{N}$ & + & $\mathrm{N}$ & $\mathrm{N}$ & $\mathrm{N}$ & $\mathrm{N}$ & $\mathrm{N}$ & $\mathrm{N}$ & $\mathrm{N}$ & $\mathrm{N}$ & $\mathrm{N}$ & $\mathrm{N}$ & $\mathrm{N}$ & $\mathrm{N}$ & $\mathrm{N}$ & $\mathrm{N}$ & $\mathrm{N}$ & $\mathrm{N}$ & \\
\hline & & & & & $\mathrm{MeOH}$ & - & $\mathrm{N}$ & $\mathrm{N}$ & - & $\mathrm{N}$ & $\mathrm{N}$ & $\mathrm{N}$ & $\mathrm{N}$ & $\mathrm{N}$ & $\mathrm{N}$ & $\mathrm{N}$ & $\mathrm{N}$ & $\mathrm{N}$ & $\mathrm{N}$ & $\mathrm{N}$ & $\mathrm{N}$ & $\mathrm{N}$ & $\mathrm{N}$ & $\mathrm{N}$ & $\mathrm{N}$ & \\
\hline & \multirow{3}{*}{$\begin{array}{c}\text { Ceruana pratensis } \\
\text { Forssk. }\end{array}$} & \multirow{3}{*}{ Jadia } & \multirow{3}{*}{ W.P. } & \multirow{3}{*}{ M1 } & $\mathrm{CHCl}_{3}$ & $\mathrm{~N}$ & $\mathrm{~N}$ & $\mathrm{~N}$ & - & $\mathrm{N}$ & $\mathrm{N}$ & $\mathrm{N}$ & $\mathrm{N}$ & $\mathrm{N}$ & $\mathrm{N}$ & $\mathrm{N}$ & $\mathrm{N}$ & $\mathrm{N}$ & $\mathrm{N}$ & $\mathrm{N}$ & $\mathrm{N}$ & $\mathrm{N}$ & + & $\mathrm{N}$ & $\mathrm{N}$ & \multirow{9}{*}{$\begin{array}{l}\text { El-Egami et } \\
\text { al. } 2006\end{array}$} \\
\hline & & & & & $\mathrm{MeOH}$ & $\mathrm{N}$ & $\mathrm{N}$ & $\mathrm{N}$ & + & $\mathrm{N}$ & $\mathrm{N}$ & $\mathrm{N}$ & $\mathrm{N}$ & $\mathrm{N}$ & $\mathrm{N}$ & $\mathrm{N}$ & $\mathrm{N}$ & $\mathrm{N}$ & $\mathrm{N}$ & $\mathrm{N}$ & $\mathrm{N}$ & $\mathrm{N}$ & + & $\mathrm{N}$ & $\mathrm{N}$ & \\
\hline & & & & & $\mathrm{H}_{2} \mathrm{O}$ & $\mathrm{N}$ & $\mathrm{N}$ & $\mathrm{N}$ & + & $\mathrm{N}$ & $\mathrm{N}$ & $\mathrm{N}$ & $\mathrm{N}$ & $\mathrm{N}$ & $\mathrm{N}$ & $\mathrm{N}$ & $\mathrm{N}$ & $\mathrm{N}$ & $\mathrm{N}$ & $\mathrm{N}$ & $\mathrm{N}$ & $\mathrm{N}$ & - & $\mathrm{N}$ & $\mathrm{N}$ & \\
\hline & \multirow{3}{*}{$\begin{array}{c}\text { Cotula aNhemoides } \\
\text { L. }\end{array}$} & \multirow{3}{*}{ Atresh } & \multirow{3}{*}{ W.P. } & \multirow{3}{*}{ M1 } & $\mathrm{CHCl}_{3}$ & $\mathrm{~N}$ & $\mathrm{~N}$ & $\mathrm{~N}$ & + & $\mathrm{N}$ & $\mathrm{N}$ & $\mathrm{N}$ & $\mathrm{N}$ & $\mathrm{N}$ & $\mathrm{N}$ & $\mathrm{N}$ & $\mathrm{N}$ & $\mathrm{N}$ & $\mathrm{N}$ & $\mathrm{N}$ & $\mathrm{N}$ & $\mathrm{N}$ & + & $\mathrm{N}$ & $\mathrm{N}$ & \\
\hline & & & & & $\mathrm{MeOH}$ & $\mathrm{N}$ & $\mathrm{N}$ & $\mathrm{N}$ & - & $\mathrm{N}$ & $\mathrm{N}$ & $\mathrm{N}$ & $\mathrm{N}$ & $\mathrm{N}$ & $\mathrm{N}$ & $\mathrm{N}$ & $\mathrm{N}$ & $\mathrm{N}$ & $\mathrm{N}$ & $\mathrm{N}$ & $\mathrm{N}$ & $\mathrm{N}$ & + & $\mathrm{N}$ & $\mathrm{N}$ & \\
\hline & & & & & $\mathrm{H}_{2} \mathrm{O}$ & $\mathrm{N}$ & $\mathrm{N}$ & $\mathrm{N}$ & - & $\mathrm{N}$ & $\mathrm{N}$ & $\mathrm{N}$ & $\mathrm{N}$ & $\mathrm{N}$ & $\mathrm{N}$ & $\mathrm{N}$ & $\mathrm{N}$ & $\mathrm{N}$ & $\mathrm{N}$ & $\mathrm{N}$ & $\mathrm{N}$ & $\mathrm{N}$ & - & $\mathrm{N}$ & $\mathrm{N}$ & \\
\hline & \multirow{3}{*}{$\begin{array}{l}\text { Geigeria alata } \\
\text { Benth. \& Hook. }\end{array}$} & & & & $\mathrm{CHCl}_{3}$ & $\mathrm{~N}$ & $\mathrm{~N}$ & $\mathrm{~N}$ & - & $\mathrm{N}$ & $\mathrm{N}$ & $\mathrm{N}$ & $\mathrm{N}$ & $\mathrm{N}$ & $\mathrm{N}$ & $\mathrm{N}$ & $\mathrm{N}$ & $\mathrm{N}$ & $\mathrm{N}$ & $\mathrm{N}$ & $\mathrm{N}$ & $\mathrm{N}$ & + & $\mathrm{N}$ & $\mathrm{N}$ & \\
\hline & & Gudgat & W.P. & M1 & $\mathrm{MeOH}$ & $\mathrm{N}$ & $\mathrm{N}$ & $\mathrm{N}$ & + & $\mathrm{N}$ & $\mathrm{N}$ & $\mathrm{N}$ & $\mathrm{N}$ & $\mathrm{N}$ & $\mathrm{N}$ & $\mathrm{N}$ & $\mathrm{N}$ & $\mathrm{N}$ & $\mathrm{N}$ & $\mathrm{N}$ & $\mathrm{N}$ & $\mathrm{N}$ & + & $\mathrm{N}$ & $\mathrm{N}$ & \\
\hline & & & & & $\mathrm{H}_{2} \mathrm{O}$ & $\mathrm{N}$ & $\mathrm{N}$ & $\mathrm{N}$ & - & $\mathrm{N}$ & $\mathrm{N}$ & $\mathrm{N}$ & $\mathrm{N}$ & $\mathrm{N}$ & $\mathrm{N}$ & $\mathrm{N}$ & $\mathrm{N}$ & $\mathrm{N}$ & $\mathrm{N}$ & $\mathrm{N}$ & $\mathrm{N}$ & $\mathrm{N}$ & - & $\mathrm{N}$ & $\mathrm{N}$ & \\
\hline Balanitaceae & $\begin{array}{c}\text { Balanites } \\
\text { aegyptiaca (L.) Del. }\end{array}$ & Hegleig & Fr.Me & M1 & $\mathrm{MeOH}$ & + & $\mathrm{N}$ & $\mathrm{N}$ & $\mathrm{N}$ & + & + & + & $\mathrm{N}$ & $\mathrm{N}$ & $\mathrm{N}$ & $\mathrm{N}$ & $\mathrm{N}$ & $\mathrm{N}$ & $\mathrm{N}$ & $\mathrm{N}$ & $\mathrm{N}$ & $\mathrm{N}$ & $\mathrm{N}$ & $\mathrm{N}$ & $\mathrm{N}$ & $\begin{array}{l}\text { Abdallah et } \\
\text { al. } 2012\end{array}$ \\
\hline & & & & & $\mathrm{CHCl}_{3}$ & - & $\mathrm{N}$ & $\mathrm{N}$ & - & $\mathrm{N}$ & $\mathrm{N}$ & $\mathrm{N}$ & $\mathrm{N}$ & $\mathrm{N}$ & $\mathrm{N}$ & $\mathrm{N}$ & $\mathrm{N}$ & $\mathrm{N}$ & $\mathrm{N}$ & $\mathrm{N}$ & $\mathrm{N}$ & $\mathrm{N}$ & $\mathrm{N}$ & $\mathrm{N}$ & $\mathrm{N}$ & \\
\hline Bignoniaceae & Kigelia africana & Umm & Fr. & M1 & $\mathrm{MeOH}$ & - & $\mathrm{N}$ & $\mathrm{N}$ & + & $\mathrm{N}$ & $\mathrm{N}$ & $\mathrm{N}$ & $\mathrm{N}$ & $\mathrm{N}$ & $\mathrm{N}$ & $\mathrm{N}$ & $\mathrm{N}$ & $\mathrm{N}$ & $\mathrm{N}$ & $\mathrm{N}$ & $\mathrm{N}$ & $\mathrm{N}$ & $\mathrm{N}$ & $\mathrm{N}$ & $\mathrm{N}$ & $\begin{array}{l}\text { Abdalla } \\
2004\end{array}$ \\
\hline & & & & & $\mathrm{H}_{2} \mathrm{O}$ & - & $\mathrm{N}$ & $\mathrm{N}$ & - & $\mathrm{N}$ & $\mathrm{N}$ & $\mathrm{N}$ & $\mathrm{N}$ & $\mathrm{N}$ & $\mathrm{N}$ & $\mathrm{N}$ & $\mathrm{N}$ & $\mathrm{N}$ & $\mathrm{N}$ & $\mathrm{N}$ & $\mathrm{N}$ & $\mathrm{N}$ & $\mathrm{N}$ & $\mathrm{N}$ & $\mathrm{N}$ & \\
\hline Bombacaceae & $\begin{array}{c}\text { Adansonia digitata } \\
\text { L. }\end{array}$ & Tabeldi & Le. & M1 & EtOH & + & $\mathrm{N}$ & $\mathrm{N}$ & + & $\mathrm{N}$ & $\mathrm{N}$ & $\mathrm{N}$ & $\mathrm{N}$ & $\mathrm{N}$ & $\mathrm{N}$ & $\mathrm{N}$ & $\mathrm{N}$ & $\mathrm{N}$ & $\mathrm{N}$ & $\mathrm{N}$ & $\mathrm{N}$ & $\mathrm{N}$ & $\mathrm{N}$ & $\mathrm{N}$ & $\mathrm{N}$ & $\begin{array}{c}\text { Kabbashi } \text { et } \\
\text { al. } 2014\end{array}$ \\
\hline & & & Le. & M1 & $\mathrm{MeOH}$ & + & $\mathrm{N}$ & $\mathrm{N}$ & + & $\mathrm{N}$ & $\mathrm{N}$ & $\mathrm{N}$ & $\mathrm{N}$ & $\mathrm{N}$ & $\mathrm{N}$ & $\mathrm{N}$ & $\mathrm{N}$ & $\mathrm{N}$ & $\mathrm{N}$ & $\mathrm{N}$ & $\mathrm{N}$ & $\mathrm{N}$ & $\mathrm{N}$ & $\mathrm{N}$ & $\mathrm{N}$ & \\
\hline & & & & & $\begin{array}{l}\text { Pet. } \\
\text { ether }\end{array}$ & - & $\mathrm{N}$ & $\mathrm{N}$ & - & $\mathrm{N}$ & $\mathrm{N}$ & $\mathrm{N}$ & $\mathrm{N}$ & $\mathrm{N}$ & $\mathrm{N}$ & $\mathrm{N}$ & $\mathrm{N}$ & $\mathrm{N}$ & $\mathrm{N}$ & $\mathrm{N}$ & $\mathrm{N}$ & $\mathrm{N}$ & $\mathrm{N}$ & $\mathrm{N}$ & $\mathrm{N}$ & \\
\hline & & & Le. & M1 & $\mathrm{CHCl}_{3}$ & - & $\mathrm{N}$ & $\mathrm{N}$ & - & $\mathrm{N}$ & $\mathrm{N}$ & $\mathrm{N}$ & $\mathrm{N}$ & $\mathrm{N}$ & $\mathrm{N}$ & $\mathrm{N}$ & $\mathrm{N}$ & $\mathrm{N}$ & $\mathrm{N}$ & $\mathrm{N}$ & $\mathrm{N}$ & $\mathrm{N}$ & $\mathrm{N}$ & $\mathrm{N}$ & $\mathrm{N}$ & \\
\hline & & & & & EtOAc & + & $\mathrm{N}$ & $\mathrm{N}$ & + & $\mathrm{N}$ & $\mathrm{N}$ & $\mathrm{N}$ & $\mathrm{N}$ & $\mathrm{N}$ & $\mathrm{N}$ & $\mathrm{N}$ & $\mathrm{N}$ & $\mathrm{N}$ & $\mathrm{N}$ & $\mathrm{N}$ & $\mathrm{N}$ & $\mathrm{N}$ & $\mathrm{N}$ & $\mathrm{N}$ & $\mathrm{N}$ & \\
\hline Boraginaceae & $\begin{array}{l}\text { Coraia ajricana } \\
\text { Lam. }\end{array}$ & Andrab & & & $\mathrm{H}_{2} \mathrm{O}$ & - & $\mathrm{N}$ & $\mathrm{N}$ & - & $\mathrm{N}$ & $\mathrm{N}$ & $\mathrm{N}$ & $\mathrm{N}$ & $\mathrm{N}$ & $\mathrm{N}$ & $\mathrm{N}$ & $\mathrm{N}$ & $\mathrm{N}$ & $\mathrm{N}$ & $\mathrm{N}$ & $\mathrm{N}$ & $\mathrm{N}$ & $\mathrm{N}$ & $\mathrm{N}$ & $\mathrm{N}$ & 2015 \\
\hline & & & Fr. & M1 & $\mathrm{MeOH}$ & + & $\mathrm{N}$ & $\mathrm{N}$ & + & $\mathrm{N}$ & $\mathrm{N}$ & $\mathrm{N}$ & $\mathrm{N}$ & $\mathrm{N}$ & $\mathrm{N}$ & $\mathrm{N}$ & $\mathrm{N}$ & $\mathrm{N}$ & $\mathrm{N}$ & $\mathrm{N}$ & $\mathrm{N}$ & $\mathrm{N}$ & $\mathrm{N}$ & $\mathrm{N}$ & $\mathrm{N}$ & \\
\hline & & & Fr. & M1 & $\begin{array}{l}\text { Pet. } \\
\text { ether }\end{array}$ & - & $\mathrm{N}$ & $\mathrm{N}$ & - & $\mathrm{N}$ & $\mathrm{N}$ & $\mathrm{N}$ & $\mathrm{N}$ & $\mathrm{N}$ & $\mathrm{N}$ & $\mathrm{N}$ & $\mathrm{N}$ & $\mathrm{N}$ & $\mathrm{N}$ & $\mathrm{N}$ & $\mathrm{N}$ & $\mathrm{N}$ & $\mathrm{N}$ & $\mathrm{N}$ & $\mathrm{N}$ & \\
\hline & & & & & $\mathrm{MeOH}$ & + & $\mathrm{N}$ & $\mathrm{N}$ & + & $\mathrm{N}$ & $\mathrm{N}$ & $\mathrm{N}$ & $\mathrm{N}$ & $\mathrm{N}$ & $\mathrm{N}$ & $\mathrm{N}$ & $\mathrm{N}$ & $\mathrm{N}$ & $\mathrm{N}$ & $\mathrm{N}$ & $\mathrm{N}$ & $\mathrm{N}$ & $\mathrm{N}$ & $\mathrm{N}$ & $\mathrm{N}$ & \\
\hline Boraginaceae & $\begin{array}{l}\text { Cordia africana } \\
\text { Lam. }\end{array}$ & Andrab & St. & M1 & $\begin{array}{l}\text { Pet. } \\
\text { ether }\end{array}$ & - & $\mathrm{N}$ & $\mathrm{N}$ & 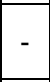 & $\mathrm{N}$ & $\mathrm{N}$ & $\mathrm{N}$ & $\mathrm{N}$ & $\mathrm{N}$ & $\mathrm{N}$ & $\mathrm{N}$ & $\mathrm{N}$ & $\mathrm{N}$ & $\mathrm{N}$ & $\mathrm{N}$ & $\mathrm{N}$ & $\mathrm{N}$ & $\mathrm{N}$ & $\mathrm{N}$ & $\mathrm{N}$ & $\begin{array}{l}\text { Alhadi et al. } \\
\quad 2015\end{array}$ \\
\hline & & & & & $\mathrm{CHCl}_{3}$ & - & $\mathrm{N}$ & $\mathrm{N}$ & - & $\mathrm{N}$ & $\mathrm{N}$ & $\mathrm{N}$ & $\mathrm{N}$ & $\mathrm{N}$ & $\mathrm{N}$ & $\mathrm{N}$ & $\mathrm{N}$ & $\mathrm{N}$ & $\mathrm{N}$ & $\mathrm{N}$ & $\mathrm{N}$ & $\mathrm{N}$ & $\mathrm{N}$ & $\mathrm{N}$ & $\mathrm{N}$ & \\
\hline
\end{tabular}




\begin{tabular}{|c|c|c|c|c|c|c|c|c|c|c|c|c|c|c|c|c|c|c|c|c|c|c|c|c|c|c|}
\hline Plant Family & $\begin{array}{l}\text { Plant Scientific } \\
\text { Name }\end{array}$ & \multirow[t]{5}{*}{$\begin{array}{c}\text { Plant } \\
\text { Vernacular } \\
\text { Name }\end{array}$} & $\begin{array}{c}\text { Plant } \\
\text { Part } \\
\end{array}$ & $\begin{array}{c}\text { Method } \\
\text { used } \\
\end{array}$ & \multirow[t]{2}{*}{$\begin{array}{c}\text { Solvent } \\
\text { used }\end{array}$} & 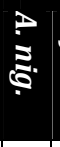 & 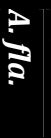 & $\stackrel{+}{\frac{1}{5}}$ & $\stackrel{?}{2}$ & 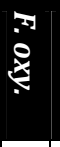 & $\stackrel{-1}{\vdots}$ & $\frac{\pi}{8}$ & $\begin{array}{l}\text { Acti } \\
: 0 \\
\tilde{\sigma}\end{array}$ & ivity & 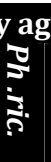 & 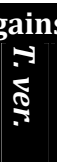 & $\begin{array}{c}\text { st fu } \\
-1 \\
\vdots \\
\vdots\end{array}$ & $\begin{array}{l}\text { ungi } \\
1 \\
5 \\
0 \\
0\end{array}$ & i & 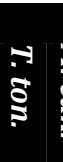 & $\underset{\mathfrak{S}}{\mathfrak{s}}$ & 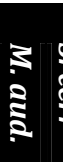 & $\stackrel{5}{9}$ & $\frac{6}{4}$ & $\underset{\substack{3 \\
3}}{3}$ & References \\
\hline \multirow{17}{*}{$\begin{array}{l}\text { Brassicaceae/ } \\
\text { Cruciferae }\end{array}$} & & & & & & + & $\mathrm{N}$ & $\mathrm{N}$ & + & $\mathrm{N}$ & $\mathrm{N}$ & $\mathrm{N}$ & $\mathrm{N}$ & $\mathrm{N}$ & $\mathrm{N}$ & $\mathrm{N}$ & $\mathrm{N}$ & $\mathrm{N}$ & $\mathrm{N}$ & $\mathrm{N}$ & $\mathrm{N}$ & $\mathrm{N}$ & $\mathrm{N}$ & $\mathrm{N}$ & $\mathrm{N}$ & \\
\hline & & & St. & M1 & $\mathrm{H}_{2} \mathrm{O}$ & - & $\mathrm{N}$ & $\mathrm{N}$ & - & $\mathrm{N}$ & $\mathrm{N}$ & $\mathrm{N}$ & $\mathrm{N}$ & $\mathrm{N}$ & $\mathrm{N}$ & $\mathrm{N}$ & $\mathrm{N}$ & $\mathrm{N}$ & $\mathrm{N}$ & $\mathrm{N}$ & $\mathrm{N}$ & $\mathrm{N}$ & $\mathrm{N}$ & $\mathrm{N}$ & $\mathrm{N}$ & \\
\hline & & & \multirow[b]{2}{*}{ Ba. } & \multirow[b]{2}{*}{ M1 } & $\mathrm{MeOH}$ & + & $\mathrm{N}$ & $\mathrm{N}$ & + & $\mathrm{N}$ & $\mathrm{N}$ & $\mathrm{N}$ & $\mathrm{N}$ & $\mathrm{N}$ & $\mathrm{N}$ & $\mathrm{N}$ & $\mathrm{N}$ & $\mathrm{N}$ & $\mathrm{N}$ & $\mathrm{N}$ & $\mathrm{N}$ & $\mathrm{N}$ & $\mathrm{N}$ & $\mathrm{N}$ & $\mathrm{N}$ & \\
\hline & & & & & $\begin{array}{l}\text { Pet. } \\
\text { ether }\end{array}$ & - & $\mathrm{N}$ & $\mathrm{N}$ & - & $\mathrm{N}$ & $\mathrm{N}$ & $\mathrm{N}$ & $\mathrm{N}$ & $\mathrm{N}$ & $\mathrm{N}$ & $\mathrm{N}$ & $\mathrm{N}$ & $\mathrm{N}$ & $\mathrm{N}$ & $\mathrm{N}$ & $\mathrm{N}$ & $\mathrm{N}$ & $\mathrm{N}$ & $\mathrm{N}$ & $\mathrm{N}$ & \\
\hline & \multirow{6}{*}{$\begin{array}{c}\text { Lepidium sativum } \\
\text { L. }\end{array}$} & \multirow{6}{*}{ El Rashad } & \multirow{6}{*}{ Se. } & \multirow{6}{*}{ M1 } & $\mathrm{MeOH}$ & \begin{tabular}{|l|}
- \\
\end{tabular} & $\mathrm{N}$ & $\mathrm{N}$ & + & $\mathrm{N}$ & $\mathrm{N}$ & $\mathrm{N}$ & $\mathrm{N}$ & $\mathrm{N}$ & $\mathrm{N}$ & $\mathrm{N}$ & $\mathrm{N}$ & $\mathrm{N}$ & $\mathrm{N}$ & $\mathrm{N}$ & \begin{tabular}{l|l}
$\mathrm{N}$ \\
\end{tabular} & $\mathrm{N}$ & $\mathrm{N}$ & $\mathrm{N}$ & $\mathrm{N}$ & \multirow{3}{*}{$\begin{array}{l}\text { Abuelgasim } \\
\text { et al. } 2015\end{array}$} \\
\hline & & & & & $\mathrm{CHCl}_{3}$ & - & $\mathrm{N}$ & $\mathrm{N}$ & - & $\mathrm{N}$ & $\mathrm{N}$ & $\mathrm{N}$ & $\mathrm{N}$ & $\mathrm{N}$ & $\mathrm{N}$ & $\mathrm{N}$ & $\mathrm{N}$ & $\mathrm{N}$ & $\mathrm{N}$ & $\mathrm{N}$ & $\mathrm{N}$ & $\mathrm{N}$ & $\mathrm{N}$ & $\mathrm{N}$ & $\mathrm{N}$ & \\
\hline & & & & & $\mathrm{H}_{2} \mathrm{O}$ & - & $\mathrm{N}$ & $\mathrm{N}$ & - & $\mathrm{N}$ & $\mathrm{N}$ & $\mathrm{N}$ & $\mathrm{N}$ & $\mathrm{N}$ & $\mathrm{N}$ & \begin{tabular}{|l|}
$\mathrm{N}$ \\
\end{tabular} & $\mathrm{N}$ & $\mathrm{N}$ & $\mathrm{N}$ & $\mathrm{N}$ & $\mathrm{N}$ & $\mathrm{N}$ & $\mathrm{N}$ & $\mathrm{N}$ & $\mathrm{N}$ & \\
\hline & & & & & $\begin{array}{l}\text { Pet. } \\
\text { ether }\end{array}$ & $\mathrm{N}$ & $\mathrm{N}$ & $\mathrm{N}$ & + & $\mathrm{N}$ & $\mathrm{N}$ & $\mathrm{N}$ & $\mathrm{N}$ & $\mathrm{N}$ & $\mathrm{N}$ & $\mathrm{N}$ & $\mathrm{N}$ & $\mathrm{N}$ & $\mathrm{N}$ & $\mathrm{N}$ & $\mathrm{N}$ & $\mathrm{N}$ & $\mathrm{N}$ & $\mathrm{N}$ & $\mathrm{N}$ & Adam et al. \\
\hline & & & & & $\mathrm{MeOH}$ & $\mathrm{N}$ & $\mathrm{N}$ & $\mathrm{N}$ & + & $\mathrm{N}$ & $\mathrm{N}$ & $\mathrm{N}$ & $\mathrm{N}$ & $\mathrm{N}$ & $\mathrm{N}$ & $\mathrm{N}$ & $\mathrm{N}$ & $\mathrm{N}$ & $\mathrm{N}$ & $\mathrm{N}$ & $\mathrm{N}$ & $\mathrm{N}$ & $\mathrm{N}$ & $\mathrm{N}$ & $\mathrm{N}$ & 2011 \\
\hline & & & & & $\mathrm{H}_{2} \mathrm{O}$ & $\mathrm{N}$ & $\mathrm{N}$ & $\mathrm{N}$ & - & $\mathrm{N}$ & $\mathrm{N}$ & $\mathrm{N}$ & $\mathrm{N}$ & $\mathrm{N}$ & $\mathrm{N}$ & $\mathrm{N}$ & $\mathrm{N}$ & $\mathrm{N}$ & $\mathrm{N}$ & $\mathrm{N}$ & $\mathrm{N}$ & $\mathrm{N}$ & $\mathrm{N}$ & $\mathrm{N}$ & $\mathrm{N}$ & \\
\hline & $\begin{array}{c}\text { Lepidium } \\
\text { virginicum } \mathrm{L} .\end{array}$ & $\begin{array}{l}\text { Hab El- } \\
\text { rashad }\end{array}$ & Se. & M1 & EtOH & $\mathrm{N}$ & $\mathrm{N}$ & $\mathrm{N}$ & + & $\mathrm{N}$ & $\mathrm{N}$ & $\mathrm{N}$ & $\mathrm{N}$ & $\mathrm{N}$ & $\mathrm{N}$ & $\mathrm{N}$ & $\mathrm{N}$ & $\mathrm{N}$ & $\mathrm{N}$ & $\mathrm{N}$ & $\mathrm{N}$ & $\mathrm{N}$ & $\mathrm{N}$ & $\mathrm{N}$ & $\mathrm{N}$ & $\begin{array}{l}\text { Sirag et al. } \\
2009\end{array}$ \\
\hline & & & & & $\mathrm{CHCl}_{3}$ & + & $\mathrm{N}$ & $\mathrm{N}$ & - & $\mathrm{N}$ & $\mathrm{N}$ & $\mathrm{N}$ & $\mathrm{N}$ & $\mathrm{N}$ & $\mathrm{N}$ & $\mathrm{N}$ & $\mathrm{N}$ & $\mathrm{N}$ & $\mathrm{N}$ & $\mathrm{N}$ & $\mathrm{N}$ & $\mathrm{N}$ & $\mathrm{N}$ & \begin{tabular}{l|l}
$\mathrm{N}$ \\
\end{tabular} & $\mathrm{N}$ & \\
\hline & Raphanus sativus L. & Figl & Se. & M1 & $\mathrm{MeOH}$ & + & $\mathrm{N}$ & $\mathrm{N}$ & + & $\mathrm{N}$ & $\mathrm{N}$ & $\mathrm{N}$ & $\mathrm{N}$ & $\mathrm{N}$ & $\mathrm{N}$ & $\mathrm{N}$ & $\mathrm{N}$ & $\mathrm{N}$ & $\mathrm{N}$ & $\mathrm{N}$ & $\mathrm{N}$ & $\mathrm{N}$ & $\mathrm{N}$ & $\mathrm{N}$ & $\mathrm{N}$ & $\begin{array}{l}\text { Abdalla } \\
2004\end{array}$ \\
\hline & & & & & $\mathrm{H}_{2} \mathrm{O}$ & - & $\mathrm{N}$ & $\mathrm{N}$ & + & $\mathrm{N}$ & $\mathrm{N}$ & $\mathrm{N}$ & $\mathrm{N}$ & $\mathrm{N}$ & $\mathrm{N}$ & $\mathrm{N}$ & $\mathrm{N}$ & $\mathrm{N}$ & $\mathrm{N}$ & $\mathrm{N}$ & $\mathrm{N}$ & $\mathrm{N}$ & $\mathrm{N}$ & $\mathrm{N}$ & $\mathrm{N}$ & \\
\hline & & & & & $\mathrm{CHCl}_{3}$ & $\mathrm{~N}$ & $\mathrm{~N}$ & $\mathrm{~N}$ & - & $\mathrm{N}$ & $\mathrm{N}$ & $\mathrm{N}$ & $\mathrm{N}$ & $\mathrm{N}$ & $\mathrm{N}$ & $\mathrm{N}$ & $\mathrm{N}$ & $\mathrm{N}$ & \begin{tabular}{|l|}
$\mathrm{N}$ \\
\end{tabular} & $\mathrm{N}$ & $\mathrm{N}$ & $\mathrm{N}$ & - & $\mathrm{N}$ & $\mathrm{N}$ & \\
\hline & $\begin{array}{c}\text { Rorippa indica (L.) } \\
\text { Hiern }\end{array}$ & Fikki & W.P. & M1 & $\mathrm{MeOH}$ & $\mathrm{N}$ & $\mathrm{N}$ & $\mathrm{N}$ & - & $\mathrm{N}$ & $\mathrm{N}$ & $\mathrm{N}$ & $\mathrm{N}$ & $\mathrm{N}$ & $\mathrm{N}$ & $\mathrm{N}$ & $\mathrm{N}$ & $\mathrm{N}$ & $\mathrm{N}$ & $\mathrm{N}$ & \begin{tabular}{l|l} 
\\
$\mathrm{N}$
\end{tabular} & $\mathrm{N}$ & + & $\mathrm{N}$ & $\mathrm{N}$ & $\begin{array}{l}\text { El-Egami et } \\
\text { al } 2006\end{array}$ \\
\hline & & & & & $\mathrm{H}_{2} \mathrm{O}$ & $\mathrm{N}$ & $\mathrm{N}$ & $\mathrm{N}$ & - & $\mathrm{N}$ & $\mathrm{N}$ & $\mathrm{N}$ & $\mathrm{N}$ & $\mathrm{N}$ & $\mathrm{N}$ & $\mathrm{N}$ & $\mathrm{N}$ & $\mathrm{N}$ & $\mathrm{N}$ & $\mathrm{N}$ & $\mathrm{N}$ & $\mathrm{N}$ & - & $\mathrm{N}$ & $\mathrm{N}$ & \\
\hline & & & & M1 & Ess. oil & + & $\mathrm{N}$ & $\mathrm{N}$ & + & $\mathrm{N}$ & $\mathrm{N}$ & $\mathrm{N}$ & $\mathrm{N}$ & $\mathrm{N}$ & $\mathrm{N}$ & $\mathrm{N}$ & $\mathrm{N}$ & $\mathrm{N}$ & $\mathrm{N}$ & $\mathrm{N}$ & $\mathrm{N}$ & $\mathrm{N}$ & $\mathrm{N}$ & $\mathrm{N}$ & $\mathrm{N}$ & $\begin{array}{l}\text { Abushama } \\
\text { et al. } 2013\end{array}$ \\
\hline & Boswellia & & & & $\mathrm{MeOH}$ & $\mathrm{N}$ & $\mathrm{N}$ & $\mathrm{N}$ & & $\mathrm{N}$ & $\mathrm{N}$ & $\mathrm{N}$ & $\mathrm{N}$ & $\mathrm{N}$ & $\mathrm{N}$ & $\mathrm{N}$ & $\mathrm{N}$ & $\mathrm{N}$ & $\mathrm{N}$ & $\mathrm{N}$ & $\mathrm{N}$ & $\mathrm{N}$ & $\mathrm{N}$ & $\mathrm{N}$ & + & \\
\hline & papyrifera (Caill. & Tarag Tarag & O.G.R. & & Hex. & $\mathrm{N}$ & $\mathrm{N}$ & $\mathrm{N}$ & & $\mathrm{N}$ & $\mathrm{N}$ & $\mathrm{N}$ & $\mathrm{N}$ & $\mathrm{N}$ & $\mathrm{N}$ & $\mathrm{N}$ & $\mathrm{N}$ & $\mathrm{N}$ & $\mathrm{N}$ & $\mathrm{N}$ & $\mathrm{N}$ & $\mathrm{N}$ & $\mathrm{N}$ & $\mathrm{N}$ & + & \\
\hline & ex Del.) Hochst. & & & M4 & $\begin{array}{c}\mathrm{MeOH} \\
\text { defatted }\end{array}$ & $\mathrm{N}$ & $\mathrm{N}$ & $\mathrm{N}$ & & $\mathrm{N}$ & $\mathrm{N}$ & $\mathrm{N}$ & $\mathrm{N}$ & $\mathrm{N}$ & $\mathrm{N}$ & $\mathrm{N}$ & $\mathrm{N}$ & $\mathrm{N}$ & $\mathrm{N}$ & $\mathrm{N}$ & $\mathrm{N}$ & $\mathrm{N}$ & $\mathrm{N}$ & $\mathrm{N}$ & + & 2015 \\
\hline Burseraceae & $\begin{array}{c}\text { Commiphora } \\
\text { africana (A.Rich.) } \\
\text { Endl. } \\
\end{array}$ & Gafal & O.G.R. & M1 & Ess. oil & + & $\mathrm{N}$ & $\mathrm{N}$ & + & $\mathrm{N}$ & $\mathrm{N}$ & $\mathrm{N}$ & $\mathrm{N}$ & $\mathrm{N}$ & $\mathrm{N}$ & $\mathrm{N}$ & $\mathrm{N}$ & $\mathrm{N}$ & $\mathrm{N}$ & $\mathrm{N}$ & $\mathrm{N}$ & $\mathrm{N}$ & $\mathrm{N}$ & $\mathrm{N}$ & $\mathrm{N}$ & $\begin{array}{c}\text { Gadir \& } \\
\text { Ahmed } \\
2014 \\
\end{array}$ \\
\hline & & & & & $\begin{array}{l}\text { Pet. } \\
\text { ether }\end{array}$ & + & $\mathrm{N}$ & $\mathrm{N}$ & + & $\mathrm{N}$ & $\mathrm{N}$ & $\mathrm{N}$ & $\mathrm{N}$ & $\mathrm{N}$ & $\mathrm{N}$ & $\mathrm{N}$ & $\mathrm{N}$ & $\mathrm{N}$ & $\mathrm{N}$ & $\mathrm{N}$ & $\mathrm{N}$ & $\mathrm{N}$ & $\mathrm{N}$ & $\mathrm{N}$ & $\mathrm{N}$ & Burham \\
\hline & Commiphora & & & & EtOAc & - & $\mathrm{N}$ & $\mathrm{N}$ & - & $\mathrm{N}$ & $\mathrm{N}$ & $\mathrm{N}$ & $\mathrm{N}$ & $\mathrm{N}$ & $\mathrm{N}$ & $\mathrm{N}$ & $\mathrm{N}$ & $\mathrm{N}$ & $\mathrm{N}$ & $\mathrm{N}$ & $\mathrm{N}$ & $\mathrm{N}$ & $\mathrm{N}$ & $\mathrm{N}$ & $\mathrm{N}$ & 2013 \\
\hline & myrrha (Nees) & Murr Hijazy & O.G.R. & M1 & $\mathrm{MeOH}$ & - & $\mathrm{N}$ & $\mathrm{N}$ & - & $\mathrm{N}$ & $\mathrm{N}$ & $\mathrm{N}$ & $\mathrm{N}$ & $\mathrm{N}$ & $\mathrm{N}$ & $\mathrm{N}$ & $\mathrm{N}$ & $\mathrm{N}$ & $\mathrm{N}$ & $\mathrm{N}$ & $\mathrm{N}$ & $\mathrm{N}$ & $\mathrm{N}$ & $\mathrm{N}$ & $\mathrm{N}$ & \\
\hline & Engl. & & & & Ess. oil & + & $\mathrm{N}$ & $\mathrm{N}$ & + & $\mathrm{N}$ & $\mathrm{N}$ & $\mathrm{N}$ & $\mathrm{N}$ & $\mathrm{N}$ & $\mathrm{N}$ & $\mathrm{N}$ & $\mathrm{N}$ & $\mathrm{N}$ & $\mathrm{N}$ & $\mathrm{N}$ & $\mathrm{N}$ & $\mathrm{N}$ & $\mathrm{N}$ & $\mathrm{N}$ & $\mathrm{N}$ & $\begin{array}{c}\text { Gadir \& } \\
\text { Ahmed } \\
2014\end{array}$ \\
\hline Cannabaceae & Cannabis sativa L. & Hasheesh & Se. & M1 & $\mathrm{MeOH}$ & - & $\mathrm{N}$ & $\mathrm{N}$ & - & $\mathrm{N}$ & $\mathrm{N}$ & $\mathrm{N}$ & $\mathrm{N}$ & $\mathrm{N}$ & $\mathrm{N}$ & $\mathrm{N}$ & $\mathrm{N}$ & $\mathrm{N}$ & $\mathrm{N}$ & $\mathrm{N}$ & $\mathrm{N}$ & $\mathrm{N}$ & $\mathrm{N}$ & $\mathrm{N}$ & $\mathrm{N}$ & Ali et al. \\
\hline
\end{tabular}




\begin{tabular}{|c|c|c|c|c|c|c|c|c|c|c|c|c|c|c|c|c|c|c|c|c|c|c|c|c|c|c|}
\hline Plant Family & $\begin{array}{l}\text { Plant Scientific } \\
\text { Name }\end{array}$ & $\begin{array}{c}\text { Plant } \\
\text { Vernacular } \\
\text { Name }\end{array}$ & $\begin{array}{l}\text { Plant } \\
\text { Part }\end{array}$ & $\begin{array}{c}\text { Method } \\
\text { used }\end{array}$ & $\begin{array}{c}\text { Solvent } \\
\text { used }\end{array}$ & के & $\stackrel{?}{\overrightarrow{2}}$ & $\stackrel{p}{3}$ & $\hat{2}$ & 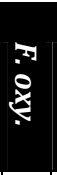 & $\stackrel{3}{3}$ & $\frac{1}{5}$ & $\begin{array}{l}\text { Act } \\
: 0 \\
5 \\
5\end{array}$ & ivity & 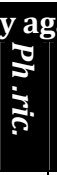 & 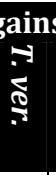 & $\begin{array}{c}\text { st fu } \\
-1 \\
\vdots \\
\vdots \\
\vdots\end{array}$ & $\begin{array}{c}\text { ungi } \\
-3 \\
5 \\
0 \\
0\end{array}$ & $\mid$ & 官 & $\begin{array}{l}\mathbf{s} \\
\hat{s}\end{array}$ & $\begin{array}{l}\mathbf{S} \\
\vdots \\
\vdots \\
\Sigma\end{array}$ & s. & $\frac{2}{2}$ & 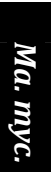 & References \\
\hline & & & \multirow[t]{2}{*}{ W.P. } & \multirow[t]{2}{*}{ M1 } & $\begin{array}{l}\text { Pet. } \\
\text { ether }\end{array}$ & - & $\mathrm{N}$ & $\mathrm{N}$ & - & $\mathrm{N}$ & $\mathrm{N}$ & $\mathrm{N}$ & $\mathrm{N}$ & $\mathrm{N}$ & $\mathrm{N}$ & $\mathrm{N}$ & $\mathrm{N}$ & $\mathrm{N}$ & $\mathrm{N}$ & $\mathrm{N}$ & $\mathrm{N}$ & $\mathrm{N}$ & $\mathrm{N}$ & $\mathrm{N}$ & $\mathrm{N}$ & \multirow[t]{2}{*}{2012} \\
\hline & & & & & $\mathrm{MeOH}$ & - & $\mathrm{N}$ & $\mathrm{N}$ & + & $\mathrm{N}$ & $\mathrm{N}$ & $\mathrm{N}$ & $\mathrm{N}$ & $\mathrm{N}$ & $\mathrm{N}$ & $\mathrm{N}$ & $\mathrm{N}$ & $\mathrm{N}$ & $\mathrm{N}$ & $\mathrm{N}$ & $\mathrm{N}$ & $\mathrm{N}$ & $\mathrm{N}$ & $\mathrm{N}$ & $\mathrm{N}$ & \\
\hline \multirow{2}{*}{$\begin{array}{l}\text { Capparaceae/ } \\
\text { Capparidaceae }\end{array}$} & \multirow{2}{*}{$\begin{array}{c}\text { Cadaba farinosa } \\
\text { Forssk. } \\
\end{array}$} & \multirow{2}{*}{$\begin{array}{l}\text { Sareh, } \\
\text { Surreh }\end{array}$} & \multirow{2}{*}{ Le. } & \multirow{2}{*}{ M1 } & $\mathrm{CHCl}_{3}$ & - & $\mathrm{N}$ & $\mathrm{N}$ & - & $\mathrm{N}$ & $\mathrm{N}$ & $\mathrm{N}$ & $\mathrm{N}$ & $\mathrm{N}$ & $\mathrm{N}$ & $\mathrm{N}$ & $\mathrm{N}$ & $\mathrm{N}$ & $\mathrm{N}$ & $\mathrm{N}$ & $\mathrm{N}$ & $\mathrm{N}$ & $\mathrm{N}$ & $\mathrm{N}$ & $\mathrm{N}$ & \multirow{2}{*}{$\begin{array}{c}\text { Moglad et } \\
\text { al. } 2012\end{array}$} \\
\hline & & & & & $\mathrm{MeOH}$ & + & $\mathrm{N}$ & $\mathrm{N}$ & - & $\mathrm{N}$ & $\mathrm{N}$ & $\mathrm{N}$ & $\mathrm{N}$ & $\mathrm{N}$ & $\mathrm{N}$ & $\mathrm{N}$ & $\mathrm{N}$ & $\mathrm{N}$ & $\mathrm{N}$ & $\mathrm{N}$ & $\mathrm{N}$ & $\mathrm{N}$ & $\mathrm{N}$ & $\mathrm{N}$ & $\mathrm{N}$ & \\
\hline \multirow{12}{*}{$\begin{array}{l}\text { Capparaceae/ } \\
\text { Capparidaceae }\end{array}$} & \multirow{3}{*}{$\begin{array}{l}\text { Capparis decidua } \\
\text { (Forssk.) Edgew. }\end{array}$} & \multirow{3}{*}{ Tundub } & \multirow{3}{*}{ St. } & \multirow{3}{*}{ M1 } & $\mathrm{CHCl}_{3}$ & + & $\mathrm{N}$ & $\mathrm{N}$ & \begin{tabular}{|l|}
+ \\
\end{tabular} & $\mathrm{N}$ & $\mathrm{N}$ & $\mathrm{N}$ & $\mathrm{N}$ & $\mathrm{N}$ & $\mathrm{N}$ & $\mathrm{N}$ & $\mathrm{N}$ & $\mathrm{N}$ & $\mathrm{N}$ & $\mathrm{N}$ & $\mathrm{N}$ & $\mathrm{N}$ & $\mathrm{N}$ & $\mathrm{N}$ & $\mathrm{N}$ & \multirow{3}{*}{$\begin{array}{c}\text { Nour \& El- } \\
\text { imam } 2013\end{array}$} \\
\hline & & & & & $\mathrm{MeOH}$ & + & $\mathrm{N}$ & $\mathrm{N}$ & + & $\mathrm{N}$ & $\mathrm{N}$ & $\mathrm{N}$ & $\mathrm{N}$ & $\mathrm{N}$ & $\mathrm{N}$ & $\mathrm{N}$ & $\mathrm{N}$ & $\mathrm{N}$ & $\mathrm{N}$ & $\mathrm{N}$ & $\mathrm{N}$ & $\mathrm{N}$ & $\mathrm{N}$ & $\mathrm{N}$ & $\mathrm{N}$ & \\
\hline & & & & & $\mathrm{H}_{2} \mathrm{O}$ & + & $\mathrm{N}$ & $\mathrm{N}$ & + & $\mathrm{N}$ & $\mathrm{N}$ & $\mathrm{N}$ & $\mathrm{N}$ & $\mathrm{N}$ & $\mathrm{N}$ & $\mathrm{N}$ & $\mathrm{N}$ & $\mathrm{N}$ & $\mathrm{N}$ & $\mathrm{N}$ & $\mathrm{N}$ & $\mathrm{N}$ & $\mathrm{N}$ & $\mathrm{N}$ & $\mathrm{N}$ & \\
\hline & & & & & $\mathrm{CHCl}_{3}$ & + & $\mathrm{N}$ & $\mathrm{N}$ & \begin{tabular}{|l|}
- \\
\end{tabular} & $\mathrm{N}$ & $\mathrm{N}$ & $\mathrm{N}$ & $\mathrm{N}$ & $\mathrm{N}$ & $\mathrm{N}$ & $\mathrm{N}$ & $\mathrm{N}$ & $\mathrm{N}$ & $\mathrm{N}$ & $\mathrm{N}$ & $\mathrm{N}$ & $\mathrm{N}$ & $\begin{array}{ll}\mathrm{N} \\
\end{array}$ & $\mathrm{N}$ & $\mathrm{N}$ & Moglad et \\
\hline & & & & & $\mathrm{MeOH}$ & + & $\mathrm{N}$ & $\mathrm{N}$ & - & $\mathrm{N}$ & $\mathrm{N}$ & $\mathrm{N}$ & $\mathrm{N}$ & $\mathrm{N}$ & $\mathrm{N}$ & $\mathrm{N}$ & $\mathrm{N}$ & $\mathrm{N}$ & $\mathrm{N}$ & $\mathrm{N}$ & $\mathrm{N}$ & $\mathrm{N}$ & $\mathrm{N}$ & $\mathrm{N}$ & $\mathrm{N}$ & al. 2012 \\
\hline & $\begin{array}{c}\text { Maerua } \\
\text { Mhlonaifolia }\end{array}$ & Abu Tamra, & Le. & M1 & $\mathrm{CHCl}_{3}$ & + & $\mathrm{N}$ & $\mathrm{N}$ & - & $\mathrm{N}$ & $\mathrm{N}$ & $\mathrm{N}$ & $\mathrm{N}$ & $\mathrm{N}$ & $\mathrm{N}$ & $\mathrm{N}$ & $\mathrm{N}$ & $\mathrm{N}$ & $\mathrm{N}$ & $\mathrm{N}$ & $\mathrm{N}$ & $\mathrm{N}$ & $\mathrm{N}$ & $\mathrm{N}$ & $\mathrm{N}$ & \\
\hline & $\begin{array}{l}\text { oblongifolia } \\
\text { Forssk A B }\end{array}$ & Surreih & & & $\mathrm{MeOH}$ & + & $\mathrm{N}$ & $\mathrm{N}$ & - & $\mathrm{N}$ & $\mathrm{N}$ & $\mathrm{N}$ & $\mathrm{N}$ & $\mathrm{N}$ & $\mathrm{N}$ & $\mathrm{N}$ & $\mathrm{N}$ & $\mathrm{N}$ & $\mathrm{N}$ & $\mathrm{N}$ & $\mathrm{N}$ & $\mathrm{N}$ & $\mathrm{N}$ & $\mathrm{N}$ & $\mathrm{N}$ & Moglad et \\
\hline & & & & & $\mathrm{CHCl}_{3}$ & + & $\mathrm{N}$ & $\mathrm{N}$ & + & $\mathrm{N}$ & $\mathrm{N}$ & $\mathrm{N}$ & $\mathrm{N}$ & $\mathrm{N}$ & $\mathrm{N}$ & $\mathrm{N}$ & $\mathrm{N}$ & $\mathrm{N}$ & $\mathrm{N}$ & $\mathrm{N}$ & $\mathrm{N}$ & $\mathrm{N}$ & $\mathrm{N}$ & $\mathrm{N}$ & $\mathrm{N}$ & al. 2014 \\
\hline & & & st. & IVI & $\mathrm{MeOH}$ & + & $\mathrm{N}$ & $\mathrm{N}$ & + & $\mathrm{N}$ & $\mathrm{N}$ & $\mathrm{N}$ & $\mathrm{N}$ & $\mathrm{N}$ & $\mathrm{N}$ & $\mathrm{N}$ & $\mathrm{N}$ & $\mathrm{N}$ & $\mathrm{N}$ & $\mathrm{N}$ & $\mathrm{N}$ & $\mathrm{N}$ & $\mathrm{N}$ & $\mathrm{N}$ & $\mathrm{N}$ & \\
\hline & Maerua & & & & $\mathrm{CHCl}_{3}$ & - & $\mathrm{N}$ & $\mathrm{N}$ & \begin{tabular}{|l|}
- \\
\end{tabular} & $\mathrm{N}$ & $\mathrm{N}$ & $\mathrm{N}$ & $\mathrm{N}$ & $\mathrm{N}$ & $\mathrm{N}$ & $\mathrm{N}$ & $\mathrm{N}$ & $\mathrm{N}$ & $\mathrm{N}$ & $\mathrm{N}$ & $\mathrm{N}$ & $\mathrm{N}$ & $\mathrm{N}$ & $\mathrm{N}$ & $\mathrm{N}$ & \\
\hline & pseudopetalosa & & & & $\mathrm{MeOH}$ & - & $\mathrm{N}$ & $\mathrm{N}$ & - & $\mathrm{N}$ & $\mathrm{N}$ & $\mathrm{N}$ & $\mathrm{N}$ & $\mathrm{N}$ & $\mathrm{N}$ & $\mathrm{N}$ & $\mathrm{N}$ & $\mathrm{N}$ & $\mathrm{N}$ & $\mathrm{N}$ & $\mathrm{N}$ & $\mathrm{N}$ & $\mathrm{N}$ & $\mathrm{N}$ & $\mathrm{N}$ & \\
\hline & $\begin{array}{l}\text { (Gilg \& Gilg-Ben.) } \\
\text { DeWolf (Courbonia } \\
\text { virgata Brongn.) }\end{array}$ & Kordala & Ro. & M1 & $\mathrm{H}_{2} \mathrm{O}$ & + & $\mathrm{N}$ & $\mathrm{N}$ & - & $\mathrm{N}$ & $\mathrm{N}$ & $\mathrm{N}$ & $\mathrm{N}$ & $\mathrm{N}$ & $\mathrm{N}$ & $\mathrm{N}$ & $\mathrm{N}$ & $\mathrm{N}$ & $\mathrm{N}$ & $\mathrm{N}$ & $\mathrm{N}$ & $\mathrm{N}$ & $\mathrm{N}$ & $\mathrm{N}$ & $\mathrm{N}$ & 2004 \\
\hline & & & & & $\mathrm{MeOH}$ & + & $\mathrm{N}$ & $\mathrm{N}$ & + & $\mathrm{N}$ & $\mathrm{N}$ & $\mathrm{N}$ & $\mathrm{N}$ & $\mathrm{N}$ & $\mathrm{N}$ & $\mathrm{N}$ & $\mathrm{N}$ & $\mathrm{N}$ & $\mathrm{N}$ & $\mathrm{N}$ & $\mathrm{N}$ & $\mathrm{N}$ & $\mathrm{N}$ & $\mathrm{N}$ & $\mathrm{N}$ & \\
\hline & & & Le. & M1 & $\begin{array}{l}\text { Pet. } \\
\text { ether }\end{array}$ & - & $\mathrm{N}$ & $\mathrm{N}$ & + & $\mathrm{N}$ & $\mathrm{N}$ & $\mathrm{N}$ & $\mathrm{N}$ & $\mathrm{N}$ & $\mathrm{N}$ & $\mathrm{N}$ & $\mathrm{N}$ & $\mathrm{N}$ & $\mathrm{N}$ & $\mathrm{N}$ & $\mathrm{N}$ & $\mathrm{N}$ & $\mathrm{N}$ & $\mathrm{N}$ & $\mathrm{N}$ & \\
\hline & & & & & $\mathrm{CHCl}_{3}$ & + & $\mathrm{N}$ & $\mathrm{N}$ & + & $\mathrm{N}$ & $\mathrm{N}$ & $\mathrm{N}$ & $\mathrm{N}$ & $\mathrm{N}$ & $\mathrm{N}$ & $\mathrm{N}$ & $\mathrm{N}$ & $\mathrm{N}$ & $\mathrm{N}$ & $\mathrm{N}$ & $\mathrm{N}$ & $\mathrm{N}$ & $\mathrm{N}$ & \begin{tabular}{|l|}
$\mathrm{N}$ \\
\end{tabular} & $\mathrm{N}$ & \\
\hline & & & Le. & M1 & EtOAc & + & $\mathrm{N}$ & $\mathrm{N}$ & + & $\mathrm{N}$ & $\mathrm{N}$ & $\mathrm{N}$ & $\mathrm{N}$ & $\mathrm{N}$ & $\mathrm{N}$ & $\mathrm{N}$ & $\mathrm{N}$ & $\mathrm{N}$ & $\mathrm{N}$ & $\mathrm{N}$ & $\mathrm{N}$ & $\mathrm{N}$ & $\mathrm{N}$ & \begin{tabular}{|l|}
$\mathrm{N}$ \\
\end{tabular} & $\mathrm{N}$ & \\
\hline & & & & & $\mathrm{MeOH}$ & + & $\mathrm{N}$ & $\mathrm{N}$ & + & $\mathrm{N}$ & $\mathrm{N}$ & $\mathrm{N}$ & $\mathrm{N}$ & $\mathrm{N}$ & $\mathrm{N}$ & $\mathrm{N}$ & $\mathrm{N}$ & $\mathrm{N}$ & $\mathrm{N}$ & $\mathrm{N}$ & $\mathrm{N}$ & $\mathrm{N}$ & $\mathrm{N}$ & \begin{tabular}{l|l}
$\mathrm{N}$ \\
\end{tabular} & $\mathrm{N}$ & \\
\hline & & & Ba. & M1 & $\begin{array}{l}\text { Pet. } \\
\text { ether }\end{array}$ & - & $\mathrm{N}$ & $\mathrm{N}$ & - & $\mathrm{N}$ & $\mathrm{N}$ & $\mathrm{N}$ & $\mathrm{N}$ & $\mathrm{N}$ & $\mathrm{N}$ & $\mathrm{N}$ & $\mathrm{N}$ & $\mathrm{N}$ & $\mathrm{N}$ & $\mathrm{N}$ & $\mathrm{N}$ & $\mathrm{N}$ & $\mathrm{N}$ & $\mathrm{N}$ & $\mathrm{N}$ & \\
\hline Combretaceae & $\begin{array}{c}\text { Anogeissus } \\
\text { leiocarpa (DC.) }\end{array}$ & Sahab & Ba. & M1 & $\mathrm{CHCl}_{3}$ & + & $\mathrm{N}$ & $\mathrm{N}$ & + & $\mathrm{N}$ & $\mathrm{N}$ & $\mathrm{N}$ & $\mathrm{N}$ & $\mathrm{N}$ & $\mathrm{N}$ & $\mathrm{N}$ & $\mathrm{N}$ & $\mathrm{N}$ & $\mathrm{N}$ & $\mathrm{N}$ & $\mathrm{N}$ & $\mathrm{N}$ & $\mathrm{N}$ & $\mathrm{N}$ & $\mathrm{N}$ & $\begin{array}{l}\text { Elsiddig et } \\
\text { al. } 2015 \mathrm{a}\end{array}$ \\
\hline & Guill. \& Perr. & & $\mathrm{Ba}$. & M1 & EtOAc & + & $\mathrm{N}$ & $\mathrm{N}$ & + & $\mathrm{N}$ & $\mathrm{N}$ & $\mathrm{N}$ & $\mathrm{N}$ & $\mathrm{N}$ & $\mathrm{N}$ & $\mathrm{N}$ & $\mathrm{N}$ & $\mathrm{N}$ & $\mathrm{N}$ & $\mathrm{N}$ & $\mathrm{N}$ & $\mathrm{N}$ & $\mathrm{N}$ & $\mathrm{N}$ & $\mathrm{N}$ & \\
\hline & & & & & $\mathrm{MeOH}$ & + & $\mathrm{N}$ & $\mathrm{N}$ & + & $\mathrm{N}$ & $\mathrm{N}$ & $\mathrm{N}$ & $\mathrm{N}$ & $\mathrm{N}$ & $\mathrm{N}$ & $\mathrm{N}$ & $\mathrm{N}$ & $\mathrm{N}$ & $\mathrm{N}$ & $\mathrm{N}$ & $\mathrm{N}$ & $\mathrm{N}$ & $\mathrm{N}$ & \begin{tabular}{|l|}
$\mathrm{N}$ \\
\end{tabular} & $\mathrm{N}$ & \\
\hline & & & Ro. & M1 & $\begin{array}{l}\text { Pet. } \\
\text { ether }\end{array}$ & - & $\mathrm{N}$ & $\mathrm{N}$ & - & $\mathrm{N}$ & $\mathrm{N}$ & $\mathrm{N}$ & $\mathrm{N}$ & $\mathrm{N}$ & $\mathrm{N}$ & $\mathrm{N}$ & $\mathrm{N}$ & $\mathrm{N}$ & $\mathrm{N}$ & $\mathrm{N}$ & $\mathrm{N}$ & $\mathrm{N}$ & $\mathrm{N}$ & $\mathrm{N}$ & $\mathrm{N}$ & \\
\hline & & & & & $\mathrm{CHCl}_{3}$ & + & $\mathrm{N}$ & $\mathrm{N}$ & + & $\mathrm{N}$ & $\mathrm{N}$ & $\mathrm{N}$ & $\mathrm{N}$ & $\mathrm{N}$ & $\mathrm{N}$ & $\mathrm{N}$ & $\mathrm{N}$ & $\mathrm{N}$ & $\mathrm{N}$ & $\mathrm{N}$ & $\mathrm{N}$ & $\mathrm{N}$ & $\mathrm{N}$ & $\mathrm{N}$ & $\mathrm{N}$ & \\
\hline & & & & & EtOAc & + & $\mathrm{N}$ & $\mathrm{N}$ & + & $\mathrm{N}$ & $\mathrm{N}$ & $\mathrm{N}$ & $\mathrm{N}$ & $\mathrm{N}$ & $\mathrm{N}$ & $\mathrm{N}$ & $\mathrm{N}$ & $\mathrm{N}$ & $\mathrm{N}$ & $\mathrm{N}$ & $\mathrm{N}$ & $\mathrm{N}$ & $\mathrm{N}$ & $\mathrm{N}$ & $\mathrm{N}$ & \\
\hline & & & Le. & M4 & $\mathrm{MeOH}$ & $\mathrm{N}$ & $\mathrm{N}$ & $\mathrm{N}$ & $\mathrm{N}$ & $\mathrm{N}$ & $\mathrm{N}$ & $\mathrm{N}$ & $\mathrm{N}$ & $\mathrm{N}$ & $\mathrm{N}$ & $\mathrm{N}$ & $\mathrm{N}$ & $\mathrm{N}$ & $\mathrm{N}$ & $\mathrm{N}$ & $\mathrm{N}$ & $\mathrm{N}$ & $\mathrm{N}$ & $\mathrm{N}$ & + & al. $2015 a$ \\
\hline
\end{tabular}




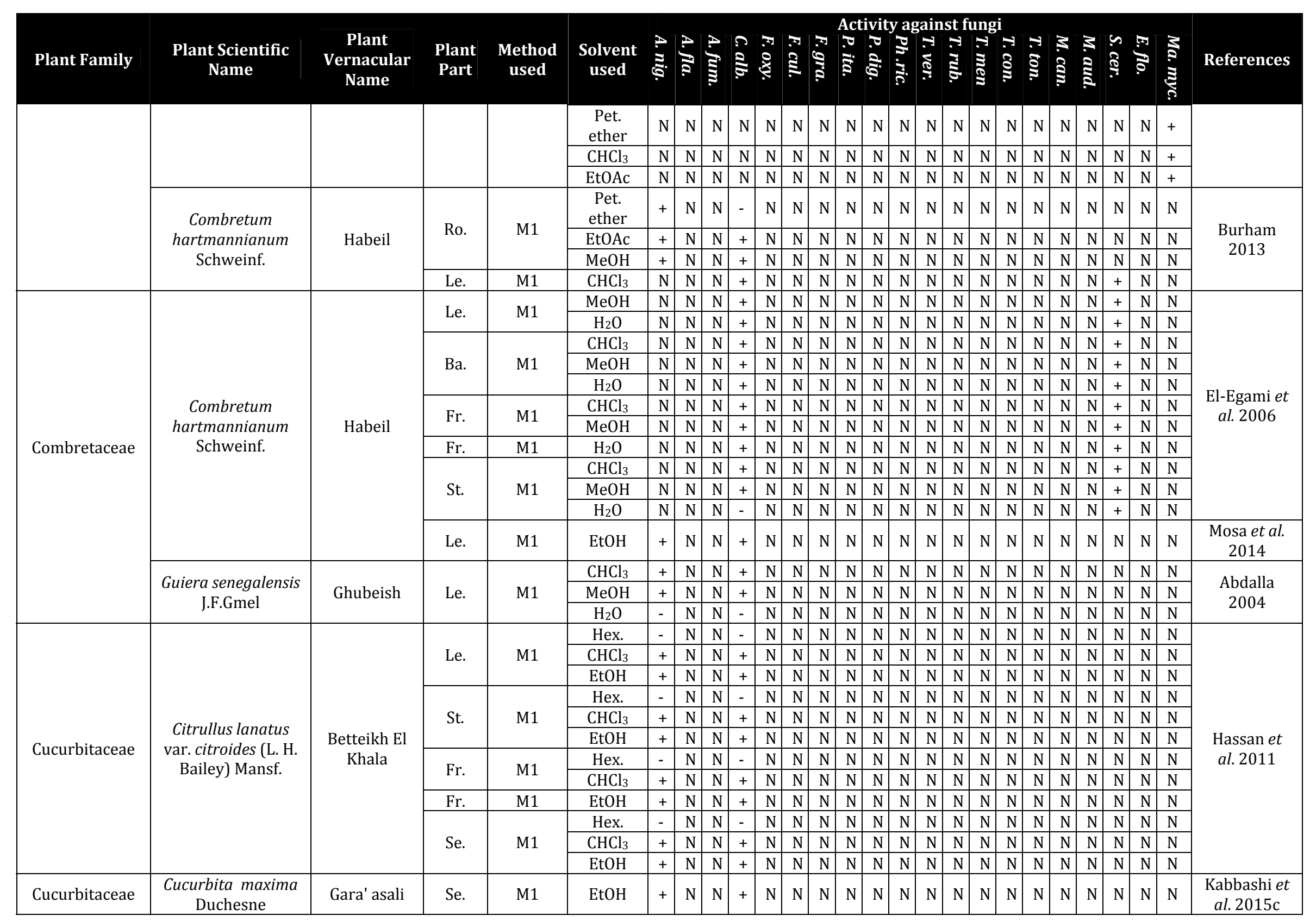




\begin{tabular}{|c|c|c|c|c|c|c|c|c|c|c|c|c|c|c|c|c|c|c|c|c|c|c|c|c|c|c|}
\hline Plant Family & $\begin{array}{l}\text { Plant Scientific } \\
\text { Name }\end{array}$ & $\begin{array}{c}\text { Plant } \\
\text { Vernacular } \\
\text { Name }\end{array}$ & $\begin{array}{l}\text { Plant } \\
\text { Part }\end{array}$ & $\begin{array}{c}\text { Method } \\
\text { used }\end{array}$ & $\begin{array}{c}\text { Solvent } \\
\text { used }\end{array}$ & क़े: & 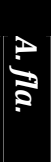 & 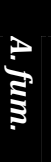 & $\hat{0}$ & 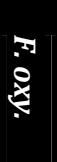 & $\stackrel{3}{3}$ & के & 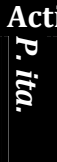 & tivity & 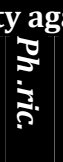 & 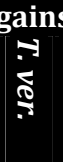 & $\mid \begin{array}{l}0 \\
\vdots \\
\vdots \\
\vdots\end{array}$ & $\mid \begin{array}{l}\text { ungi } \\
0 \\
3 \\
0 \\
0\end{array}$ & $\vec{i}$ & $\begin{array}{c}-1 \\
\hat{s}\end{array}$ & 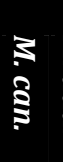 & $\mid \begin{array}{c}\mathbf{s} \\
\vdots \\
\vdots \\
\vdots\end{array}$ & $\stackrel{1}{\$}$ & $\frac{0}{2}$ & $\frac{2}{3}$ & References \\
\hline \multirow{2}{*}{ Cyperaceae } & \multirow{2}{*}{$\begin{array}{c}\text { Cyperus rotundus } \\
\text { L. }\end{array}$} & \multirow{2}{*}{ Sei'da } & Co. & M1 & Ess. oil & + & $\mathrm{N}$ & $\mathrm{N}$ & + & $\mathrm{N}$ & $\mathrm{N}$ & $\mathrm{N}$ & $\mathrm{N}$ & $\mathrm{N}$ & $\mathrm{N}$ & $\mathrm{N}$ & $\mathrm{N}$ & $\mathrm{N}$ & $\mathrm{N}$ & $\mathrm{N}$ & $\mathrm{N}$ & $\mathrm{N}$ & $\mathrm{N}$ & $\mathrm{N}$ & $\mathrm{N}$ & $\begin{array}{l}\text { Abushama } \\
\text { et al. } 2013\end{array}$ \\
\hline & & & W.P. & M1 & EtOH & + & $\mathrm{N}$ & $\mathrm{N}$ & + & $\mathrm{N}$ & $\mathrm{N}$ & $\mathrm{N}$ & $\mathrm{N}$ & $\mathrm{N}$ & $\mathrm{N}$ & $\mathrm{N}$ & $\mathrm{N}$ & $\mathrm{N}$ & $\mathrm{N}$ & $\mathrm{N}$ & $\mathrm{N}$ & $\mathrm{N}$ & $\mathrm{N}$ & $\mathrm{N}$ & $\mathrm{N}$ & $\begin{array}{c}\text { Kabbashi et } \\
\text { al. 2015c }\end{array}$ \\
\hline \multirow{6}{*}{ Ebenaceae } & \multirow{6}{*}{$\begin{array}{l}\text { Euclea racemosa L. } \\
\text { ssp. schimperi }(E . \\
\text { schimperi }\end{array}$} & \multirow{6}{*}{ Ogunim gun } & \multirow{3}{*}{ Le. } & \multirow{3}{*}{ M1 } & $\mathrm{CHCl}_{3}$ & $\mathrm{~N}$ & $\mathrm{~N}$ & $\mathrm{~N}$ & - & $\mathrm{N}$ & $\mathrm{N}$ & $\mathrm{N}$ & $\mathrm{N}$ & $\mathrm{N}$ & $\mathrm{N}$ & $\mathrm{N}$ & $\mathrm{N}$ & \begin{tabular}{l|}
$\mathrm{N}$ \\
\end{tabular} & $\mathrm{N}$ & $\mathrm{N}$ & $\mathrm{N}$ & $\mathrm{N}$ & + & $\mathrm{N}$ & $\mathrm{N}$ & \multirow{6}{*}{$\begin{array}{l}\text { El-Egami et } \\
\text { al. } 2006\end{array}$} \\
\hline & & & & & $\mathrm{MeOH}$ & $\mathrm{N}$ & $\mathrm{N}$ & $\mathrm{N}$ & + & $\mathrm{N}$ & $\mathrm{N}$ & $\mathrm{N}$ & $\mathrm{N}$ & $\mathrm{N}$ & $\mathrm{N}$ & $\mathrm{N}$ & $\mathrm{N}$ & $\mathrm{N}$ & $\mathrm{N}$ & $\mathrm{N}$ & $\mathrm{N}$ & $\mathrm{N}$ & + & $\mathrm{N}$ & $\mathrm{N}$ & \\
\hline & & & & & $\mathrm{H}_{2} \mathrm{O}$ & $\mathrm{N}$ & $\mathrm{N}$ & $\mathrm{N}$ & + & $\mathrm{N}$ & $\mathrm{N}$ & $\mathrm{N}$ & $\mathrm{N}$ & \begin{tabular}{|l|}
$\mathrm{N}$ \\
\end{tabular} & \begin{tabular}{|l|}
$\mathrm{N}$ \\
\end{tabular} & $\mathrm{N}$ & $\mathrm{N}$ & $\mathrm{N}$ & $\mathrm{N}$ & $\mathrm{N}$ & $\mathrm{N}$ & $\mathrm{N}$ & + & \begin{tabular}{|l|}
$\mathrm{N}$ \\
\end{tabular} & $\mathrm{N}$ & \\
\hline & & & \multirow{3}{*}{ St. } & \multirow{3}{*}{ M1 } & $\mathrm{CHCl}_{3}$ & $\mathrm{~N}$ & $\mathrm{~N}$ & $\mathrm{~N}$ & - & $\mathrm{N}$ & $\mathrm{N}$ & $\mathrm{N}$ & $\mathrm{N}$ & $\mathrm{N}$ & $\mathrm{N}$ & $\mathrm{N}$ & $\mathrm{N}$ & $\mathrm{N}$ & $\mathrm{N}$ & $\mathrm{N}$ & $\mathrm{N}$ & $\mathrm{N}$ & - & $\mathrm{N}$ & $\mathrm{N}$ & \\
\hline & & & & & $\mathrm{MeOH}$ & $\mathrm{N}$ & $\mathrm{N}$ & $\mathrm{N}$ & + & $\mathrm{N}$ & $\mathrm{N}$ & $\mathrm{N}$ & $\mathrm{N}$ & $\mathrm{N}$ & $\mathrm{N}$ & $\mathrm{N}$ & $\mathrm{N}$ & $\mathrm{N}$ & $\mathrm{N}$ & $\mathrm{N}$ & $\mathrm{N}$ & $\mathrm{N}$ & + & $\mathrm{N}$ & $\mathrm{N}$ & \\
\hline & & & & & $\mathrm{H}_{2} \mathrm{O}$ & $\mathrm{N}$ & $\mathrm{N}$ & $\mathrm{N}$ & + & $\mathrm{N}$ & $\mathrm{N}$ & $\mathrm{N}$ & $\mathrm{N}$ & $\mathrm{N}$ & $\mathrm{N}$ & $\mathrm{N}$ & $\mathrm{N}$ & $\mathrm{N}$ & $\mathrm{N}$ & $\mathrm{N}$ & $\mathrm{N}$ & $\mathrm{N}$ & - & \begin{tabular}{|l|}
$\mathrm{N}$ \\
\end{tabular} & $\mathrm{N}$ & \\
\hline \multirow{17}{*}{ Euphorbiaceae } & \multirow{7}{*}{$\begin{array}{c}\text { Chrozophora } \\
\text { brocchiana (Vis.) } \\
\text { Schweinf. }\end{array}$} & \multirow{7}{*}{ Tarba } & \multirow{3}{*}{ Le. } & \multirow{3}{*}{ M1 } & $\mathrm{MeOH}$ & + & $\mathrm{N}$ & $\mathrm{N}$ & + & $\mathrm{N}$ & $\mathrm{N}$ & $\mathrm{N}$ & $\mathrm{N}$ & $\mathrm{N}$ & $\mathrm{N}$ & $\mathrm{N}$ & $\mathrm{N}$ & $\mathrm{N}$ & $\mathrm{N}$ & $\mathrm{N}$ & $\mathrm{N}$ & $\mathrm{N}$ & $\mathrm{N}$ & $\mathrm{N}$ & $\mathrm{N}$ & \multirow{7}{*}{$\begin{array}{c}\text { Ahmed et al. } \\
2014\end{array}$} \\
\hline & & & & & EtOAc & - & $\mathrm{N}$ & $\mathrm{N}$ & - & $\mathrm{N}$ & $\mathrm{N}$ & $\mathrm{N}$ & $\mathrm{N}$ & $\mathrm{N}$ & $\mathrm{N}$ & $\mathrm{N}$ & $\mathrm{N}$ & $\mathrm{N}$ & $\mathrm{N}$ & $\mathrm{N}$ & $\mathrm{N}$ & $\mathrm{N}$ & $\mathrm{N}$ & $\mathrm{N}$ & $\mathrm{N}$ & \\
\hline & & & & & Hex. & - & $\mathrm{N}$ & $\mathrm{N}$ & - & $\mathrm{N}$ & $\mathrm{N}$ & $\mathrm{N}$ & $\mathrm{N}$ & $\mathrm{N}$ & $\mathrm{N}$ & $\mathrm{N}$ & $\mathrm{N}$ & $\mathrm{N}$ & $\mathrm{N}$ & $\mathrm{N}$ & $\mathrm{N}$ & $\mathrm{N}$ & $\mathrm{N}$ & $\mathrm{N}$ & $\mathrm{N}$ & \\
\hline & & & \multirow{3}{*}{ St. } & \multirow{3}{*}{ M1 } & $\mathrm{MeOH}$ & + & $\mathrm{N}$ & $\mathrm{N}$ & + & $\mathrm{N}$ & $\mathrm{N}$ & $\mathrm{N}$ & $\mathrm{N}$ & $\mathrm{N}$ & $\mathrm{N}$ & $\mathrm{N}$ & $\mathrm{N}$ & $\mathrm{N}$ & $\mathrm{N}$ & $\mathrm{N}$ & $\mathrm{N}$ & $\mathrm{N}$ & $\mathrm{N}$ & $\mathrm{N}$ & $\mathrm{N}$ & \\
\hline & & & & & EtOAc & - & $\mathrm{N}$ & $\mathrm{N}$ & - & $\mathrm{N}$ & $\mathrm{N}$ & $\mathrm{N}$ & $\mathrm{N}$ & \begin{tabular}{|l|}
$\mathrm{N}$ \\
\end{tabular} & \begin{tabular}{|l|}
$\mathrm{N}$ \\
\end{tabular} & $\mathrm{N}$ & $\mathrm{N}$ & \begin{tabular}{|l|}
$\mathrm{N}$ \\
\end{tabular} & $\mathrm{N}$ & $\mathrm{N}$ & $\mathrm{N}$ & $\mathrm{N}$ & $\mathrm{N}$ & \begin{tabular}{|l|}
$\mathrm{N}$ \\
\end{tabular} & $\mathrm{N}$ & \\
\hline & & & & & Hex. & - & $\mathrm{N}$ & $\mathrm{N}$ & - & $\mathrm{N}$ & $\mathrm{N}$ & $\mathrm{N}$ & $\mathrm{N}$ & $\mathrm{N}$ & $\mathrm{N}$ & $\mathrm{N}$ & $\mathrm{N}$ & $\mathrm{N}$ & $\mathrm{N}$ & $\mathrm{N}$ & $\mathrm{N}$ & $\mathrm{N}$ & $\mathrm{N}$ & $\mathrm{N}$ & $\mathrm{N}$ & \\
\hline & & & $\begin{array}{l}\text { Oil } \\
\text { Se. }\end{array}$ & M1 & $\mathrm{MeOH}$ & - & $\mathrm{N}$ & $\mathrm{N}$ & + & $\mathrm{N}$ & $\mathrm{N}$ & $\mathrm{N}$ & $\mathrm{N}$ & $\mathrm{N}$ & $\mathrm{N}$ & $\mathrm{N}$ & $\mathrm{N}$ & $\mathrm{N}$ & $\mathrm{N}$ & $\mathrm{N}$ & $\mathrm{N}$ & $\mathrm{N}$ & $\mathrm{N}$ & $\mathrm{N}$ & $\mathrm{N}$ & \\
\hline & & & Se. & M2 & $\begin{array}{l}\text { Pet. } \\
\text { ether }\end{array}$ & - & - & - & - & $\mathrm{N}$ & $\mathrm{N}$ & $\mathrm{N}$ & $\mathrm{N}$ & $\mathrm{N}$ & $\mathrm{N}$ & $\mathrm{N}$ & $\mathrm{N}$ & $\mathrm{N}$ & $\mathrm{N}$ & $\mathrm{N}$ & $\mathrm{N}$ & $\mathrm{N}$ & $\mathrm{N}$ & $\mathrm{N}$ & $\mathrm{N}$ & \multirow{8}{*}{$\begin{array}{c}\text { ElNour et al. } \\
2015\end{array}$} \\
\hline & & & & & $\mathrm{MeOH}$ & + & + & + & + & $\mathrm{N}$ & $\mathrm{N}$ & $\mathrm{N}$ & $\mathrm{N}$ & $\mathrm{N}$ & $\mathrm{N}$ & $\mathrm{N}$ & $\mathrm{N}$ & $\mathrm{N}$ & $\mathrm{N}$ & $\mathrm{N}$ & $\mathrm{N}$ & $\mathrm{N}$ & $\mathrm{N}$ & $\mathrm{N}$ & $\mathrm{N}$ & \\
\hline & & & Le. & M2 & $\begin{array}{c}\text { Pet. } \\
\text { ether }\end{array}$ & - & - & - & - & $\mathrm{N}$ & $\mathrm{N}$ & $\mathrm{N}$ & $\mathrm{N}$ & $\mathrm{N}$ & $\mathrm{N}$ & $\mathrm{N}$ & $\mathrm{N}$ & $\mathrm{N}$ & $\mathrm{N}$ & $\mathrm{N}$ & $\mathrm{N}$ & $\mathrm{N}$ & $\mathrm{N}$ & $\mathrm{N}$ & $\mathrm{N}$ & \\
\hline & & Habb El & & & $\mathrm{MeOH}$ & + & + & + & + & $\mathrm{N}$ & $\mathrm{N}$ & $\mathrm{N}$ & $\mathrm{N}$ & $\mathrm{N}$ & $\mathrm{N}$ & $\mathrm{N}$ & $\mathrm{N}$ & $\mathrm{N}$ & $\mathrm{N}$ & $\mathrm{N}$ & $\mathrm{N}$ & $\mathrm{N}$ & $\mathrm{N}$ & \begin{tabular}{|l|l|}
$\mathrm{N}$ \\
\end{tabular} & $\mathrm{N}$ & \\
\hline & Jatropha curcas L. & Meluk & Hy.Ca & M2 & $\begin{array}{l}\text { Pet. } \\
\text { ether }\end{array}$ & + & + & + & - & $\mathrm{N}$ & $\mathrm{N}$ & $\mathrm{N}$ & $\mathrm{N}$ & $\mathrm{N}$ & $\mathrm{N}$ & $\mathrm{N}$ & $\mathrm{N}$ & $\mathrm{N}$ & $\mathrm{N}$ & $\mathrm{N}$ & $\mathrm{N}$ & $\mathrm{N}$ & $\mathrm{N}$ & $\mathrm{N}$ & $\mathrm{N}$ & \\
\hline & & & & & $\mathrm{MeOH}$ & + & + & + & + & $\mathrm{N}$ & $\mathrm{N}$ & $\mathrm{N}$ & $\mathrm{N}$ & $\mathrm{N}$ & $\mathrm{N}$ & $\mathrm{N}$ & $\mathrm{N}$ & $\mathrm{N}$ & $\mathrm{N}$ & $\mathrm{N}$ & $\mathrm{N}$ & $\mathrm{N}$ & $\mathrm{N}$ & $\mathrm{N}$ & $\mathrm{N}$ & \\
\hline & & & Co.Ca & M2 & $\begin{array}{l}\text { Pet. } \\
\text { ether }\end{array}$ & - & + & + & - & $\mathrm{N}$ & $\mathrm{N}$ & $\mathrm{N}$ & $\mathrm{N}$ & $\mathrm{N}$ & $\mathrm{N}$ & $\mathrm{N}$ & $\mathrm{N}$ & $\mathrm{N}$ & $\mathrm{N}$ & $\mathrm{N}$ & $\mathrm{N}$ & $\mathrm{N}$ & $\mathrm{N}$ & $\mathrm{N}$ & $\mathrm{N}$ & \\
\hline & & & & & $\mathrm{MeOH}$ & + & + & + & + & $\mathrm{N}$ & $\mathrm{N}$ & $\mathrm{N}$ & $\mathrm{N}$ & $\mathrm{N}$ & $\mathrm{N}$ & $\mathrm{N}$ & $\mathrm{N}$ & $\mathrm{N}$ & $\mathrm{N}$ & $\mathrm{N}$ & $\mathrm{N}$ & $\mathrm{N}$ & $\mathrm{N}$ & $\mathrm{N}$ & $\mathrm{N}$ & \\
\hline & Ricinus communis & & Le. & & & + & $\mathrm{N}$ & $\mathrm{N}$ & + & $\mathrm{N}$ & $\mathrm{N}$ & $\mathrm{N}$ & $\mathrm{N}$ & $\mathrm{N}$ & $\mathrm{N}$ & $\mathrm{N}$ & $\mathrm{N}$ & $\mathrm{N}$ & $\mathrm{N}$ & $\mathrm{N}$ & $\mathrm{N}$ & $\mathrm{N}$ & $\mathrm{N}$ & \begin{tabular}{|l|l|}
$\mathrm{N}$ \\
\end{tabular} & $\mathrm{N}$ & Abd-Ulgadir \\
\hline & $\mathrm{I}$ & Khirwl & Se. & M1 & МeOH & - & $\mathrm{N}$ & $\mathrm{N}$ & - & $\mathrm{N}$ & $\mathrm{N}$ & $\mathrm{N}$ & $\mathrm{N}$ & $\mathrm{N}$ & $\mathrm{N}$ & $\mathrm{N}$ & $\mathrm{N}$ & $\mathrm{N}$ & $\mathrm{N}$ & $\mathrm{N}$ & $\mathrm{N}$ & $\mathrm{N}$ & $\mathrm{N}$ & $\mathrm{N}$ & $\mathrm{N}$ & et al. 2015 \\
\hline Fabaceae & Senna occidentalis & & & & $\mathrm{CHCl}_{3}$ & - & $\mathrm{N}$ & $\mathrm{N}$ & + & $\mathrm{N}$ & $\mathrm{N}$ & $\mathrm{N}$ & $\mathrm{N}$ & $\mathrm{N}$ & \begin{tabular}{|l|}
$\mathrm{N}$ \\
\end{tabular} & $\mathrm{N}$ & $\mathrm{N}$ & \begin{tabular}{|l|}
$\mathrm{N}$ \\
\end{tabular} & $\mathrm{N}$ & \begin{tabular}{|l|}
$\mathrm{N}$ \\
\end{tabular} & $\mathrm{N}$ & \begin{tabular}{|l|}
$\mathrm{N}$ \\
\end{tabular} & $\mathrm{N}$ & \begin{tabular}{|l|l|}
$\mathrm{N}$ \\
\end{tabular} & $\mathrm{N}$ & \\
\hline $\begin{array}{c}\text { (subfam. } \\
\text { Caesalpinioide } \\
\text { ae) }\end{array}$ & $\begin{array}{l}\text { (L.) Link (Cassia } \\
\text { occidentalis L.) }\end{array}$ & Soreib & Le & M1 & $\mathrm{MeOH}$ & + & $\mathrm{N}$ & $\mathrm{N}$ & + & $\mathrm{N}$ & $\mathrm{N}$ & $\mathrm{N}$ & $\mathrm{N}$ & $\mathrm{N}$ & $\mathrm{N}$ & $\mathrm{N}$ & $\mathrm{N}$ & $\mathrm{N}$ & $\mathrm{N}$ & $\mathrm{N}$ & $\mathrm{N}$ & $\mathrm{N}$ & $\mathrm{N}$ & $\mathrm{N}$ & $\mathrm{N}$ & $\begin{array}{l}\text { Moglad et } \\
\text { al. } 2012\end{array}$ \\
\hline Fabaceae & & & & & $\mathrm{CHCl}_{3}$ & $\mathrm{~N}$ & $\mathrm{~N}$ & $\mathrm{~N}$ & - & $\mathrm{N}$ & $\mathrm{N}$ & $\mathrm{N}$ & $\mathrm{N}$ & $\mathrm{N}$ & $\mathrm{N}$ & $\mathrm{N}$ & $\mathrm{N}$ & $\mathrm{N}$ & \begin{tabular}{|l|}
$\mathrm{N}$ \\
\end{tabular} & $\mathrm{N}$ & $\mathrm{N}$ & \begin{tabular}{|l|}
$\mathrm{N}$ \\
\end{tabular} & - & $\mathrm{N}$ & $\mathrm{N}$ & \\
\hline (subfam. & indica L. & - & Fr. & M1 & $\mathrm{MeOH}$ & $\mathrm{N}$ & $\mathrm{N}$ & $\mathrm{N}$ & - & $\mathrm{N}$ & $\mathrm{N}$ & $\mathrm{N}$ & $\mathrm{N}$ & $\mathrm{N}$ & $\mathrm{N}$ & $\mathrm{N}$ & $\mathrm{N}$ & $\mathrm{N}$ & $\mathrm{N}$ & $\mathrm{N}$ & $\mathrm{N}$ & $\mathrm{N}$ & + & $\mathrm{N}$ & $\mathrm{N}$ & al. 2006 \\
\hline Faboideae) & & & & & $\mathrm{H}_{2} \mathrm{O}$ & $\mathrm{N}$ & $\mathrm{N}$ & $\mathrm{N}$ & - & $\mathrm{N}$ & $\mathrm{N}$ & $\mathrm{N}$ & $\mathrm{N}$ & \begin{tabular}{|l|}
$\mathrm{N}$ \\
\end{tabular} & \begin{tabular}{|l|}
$\mathrm{N}$ \\
\end{tabular} & $\mathrm{N}$ & $\mathrm{N}$ & \begin{tabular}{|l|}
$\mathrm{N}$ \\
\end{tabular} & \begin{tabular}{|l|}
$\mathrm{N}$ \\
\end{tabular} & $\mathrm{N}$ & \begin{tabular}{|l|}
$\mathrm{N}$ \\
\end{tabular} & \begin{tabular}{|l|}
$\mathrm{N}$ \\
\end{tabular} & \begin{tabular}{|l|l|}
- & \\
\end{tabular} & \begin{tabular}{|l|l|}
$\mathrm{N}$ \\
\end{tabular} & $\mathrm{N}$ & \\
\hline
\end{tabular}




\begin{tabular}{|c|c|c|c|c|c|c|c|c|c|c|c|c|c|c|c|c|c|c|c|c|c|c|c|c|c|c|}
\hline Plant Family & $\begin{array}{c}\text { Plant Scientific } \\
\text { Name }\end{array}$ & $\begin{array}{c}\text { Plant } \\
\text { Vernacular } \\
\text { Name }\end{array}$ & $\begin{array}{l}\text { Plant } \\
\text { Part }\end{array}$ & $\begin{array}{c}\text { Method } \\
\text { used }\end{array}$ & $\begin{array}{c}\text { Solvent } \\
\text { used }\end{array}$ & $\frac{\overrightarrow{0}}{\mathrm{~s}}$ & $\stackrel{\overrightarrow{2}}{\mathfrak{R}}$ & $\stackrel{\stackrel{p}{\vec{s}}}{\mathbf{s}}$ & $\stackrel{?}{?}$ & $\vec{x}$ & $\stackrel{-1}{\vdots}$ & $\begin{array}{l}\overrightarrow{1} \\
0 \\
0 \\
0\end{array}$ & $\begin{array}{l}\text { Act } \\
: \overrightarrow{5} \\
\dot{F}\end{array}$ & tivit & 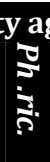 & $\begin{array}{c}\text { gain } \\
-1 \\
\vdots \\
9\end{array}$ & $\mid \begin{array}{l}n+f \\
-1 \\
\vdots \\
\vdots\end{array}$ & 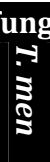 & $\dot{i}$ & $\mid \begin{array}{c}\overrightarrow{1} \\
\hat{\mathbf{s}}\end{array}$ & 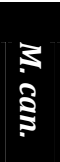 & $\begin{array}{l}\mathbf{Z} \\
\mathbf{\Xi} \\
\mathbf{\Sigma}\end{array}$ & $\stackrel{5}{\$}$ & $\frac{9}{5}$ & $\frac{\sqrt{2}}{3}$ & References \\
\hline & $\begin{array}{c}\text { Glycyrrhiza glabra } \\
\text { L. }\end{array}$ & Irgsoos & Ro. & M1 & EtOH & $\mathrm{N}$ & $\mathrm{N}$ & $\mathrm{N}$ & - & $\mathrm{N}$ & $\mathrm{N}$ & $\mathrm{N}$ & $\mathrm{N}$ & $\mathrm{N}$ & $\mathrm{N}$ & $\mathrm{N}$ & $\mathrm{N}$ & $\mathrm{N}$ & $\mathrm{N}$ & $\mathrm{N}$ & $\mathrm{N}$ & $\mathrm{N}$ & $\mathrm{N}$ & $\mathrm{N}$ & $\mathrm{N}$ & $\begin{array}{l}\text { Sirag et al. } \\
\quad 2009\end{array}$ \\
\hline & & & & & $\mathrm{CHCl}_{3}$ & $\mathrm{~N}$ & $\mathrm{~N}$ & $\mathrm{~N}$ & + & $\mathrm{N}$ & $\mathrm{N}$ & $\mathrm{N}$ & $\mathrm{N}$ & $\mathrm{N}$ & $\mathrm{N}$ & $\mathrm{N}$ & $\mathrm{N}$ & $\mathrm{N}$ & $\mathrm{N}$ & $\mathrm{N}$ & $\mathrm{N}$ & $\mathrm{N}$ & + & $\mathrm{N}$ & $\mathrm{N}$ & \\
\hline & $\begin{array}{l}\text { Inaigofera spinosa } \\
\text { Forssk }\end{array}$ & Soun'god & W.P. & M1 & $\mathrm{MeOH}$ & $\mathrm{N}$ & $\mathrm{N}$ & $\mathrm{N}$ & + & $\mathrm{N}$ & $\mathrm{N}$ & $\mathrm{N}$ & $\mathrm{N}$ & $\mathrm{N}$ & $\mathrm{N}$ & $\mathrm{N}$ & $\mathrm{N}$ & $\mathrm{N}$ & $\mathrm{N}$ & $\mathrm{N}$ & $\mathrm{N}$ & $\mathrm{N}$ & + & $\mathrm{N}$ & $\mathrm{N}$ & Elegamı et \\
\hline & & & & & $\mathrm{H}_{2} \mathrm{O}$ & $\mathrm{N}$ & $\mathrm{N}$ & $\mathrm{N}$ & - & $\mathrm{N}$ & $\mathrm{N}$ & $\mathrm{N}$ & $\mathrm{N}$ & $\mathrm{N}$ & $\mathrm{N}$ & $\mathrm{N}$ & $\mathrm{N}$ & $\mathrm{N}$ & $\mathrm{N}$ & $\mathrm{N}$ & $\mathrm{N}$ & $\mathrm{N}$ & - & $\mathrm{N}$ & $\mathrm{N}$ & \\
\hline & Lupinus albus L. (L. & Turmus & Ro. & M1 & $\begin{array}{l}\text { Pet. } \\
\text { ether }\end{array}$ & - & $\mathrm{N}$ & $\mathrm{N}$ & + & $\mathrm{N}$ & $\mathrm{N}$ & $\mathrm{N}$ & $\mathrm{N}$ & $\mathrm{N}$ & $\mathrm{N}$ & $\mathrm{N}$ & $\mathrm{N}$ & $\mathrm{N}$ & $\mathrm{N}$ & $\mathrm{N}$ & $\mathrm{N}$ & $\mathrm{N}$ & $\mathrm{N}$ & $\mathrm{N}$ & $\mathrm{N}$ & Hamed \& \\
\hline & & & & & $\mathrm{MeOH}$ & + & $\mathrm{N}$ & $\mathrm{N}$ & - & $\mathrm{N}$ & $\mathrm{N}$ & $\mathrm{N}$ & $\mathrm{N}$ & $\mathrm{N}$ & $\mathrm{N}$ & $\mathrm{N}$ & $\mathrm{N}$ & $\mathrm{N}$ & $\mathrm{N}$ & $\mathrm{N}$ & $\mathrm{N}$ & $\mathrm{N}$ & $\mathrm{N}$ & $\mathrm{N}$ & $\mathrm{N}$ & \\
\hline & $\begin{array}{l}\text { Trigonella foenum- } \\
\text { graecum L. }\end{array}$ & Helba & Se. & M1 & Seed oil & + & $\mathrm{N}$ & $\mathrm{N}$ & $\mathrm{N}$ & $\mathrm{N}$ & $\mathrm{N}$ & $\mathrm{N}$ & $\mathrm{N}$ & $\mathrm{N}$ & $\mathrm{N}$ & $\mathrm{N}$ & $\mathrm{N}$ & $\mathrm{N}$ & $\mathrm{N}$ & $\mathrm{N}$ & $\mathrm{N}$ & $\mathrm{N}$ & $\mathrm{N}$ & $\mathrm{N}$ & $\mathrm{N}$ & $\begin{array}{l}\text { Sulieman } \text { et } \\
\text { al. } 2008\end{array}$ \\
\hline & $\begin{array}{c}\text { Sesbania } \\
\text { leptocarpa DC. }\end{array}$ & Surib & Se. & M1 & EtOH & - & $\mathrm{N}$ & $\mathrm{N}$ & - & $\mathrm{N}$ & $\mathrm{N}$ & $\mathrm{N}$ & $\mathrm{N}$ & $\mathrm{N}$ & $\mathrm{N}$ & $\mathrm{N}$ & $\mathrm{N}$ & $\mathrm{N}$ & $\mathrm{N}$ & $\mathrm{N}$ & $\mathrm{N}$ & $\mathrm{N}$ & $\mathrm{N}$ & $\mathrm{N}$ & $\mathrm{N}$ & $\begin{array}{l}\text { Mosa et al. } \\
2014\end{array}$ \\
\hline \multirow{23}{*}{$\begin{array}{c}\text { Fabaceae } \\
\text { (subfam. } \\
\text { Mimosoideae) }\end{array}$} & \multirow{23}{*}{$\begin{array}{c}\text { Acacia nilotica (L.) } \\
\text { Del. }\end{array}$} & \multirow{23}{*}{ Sunt } & Po. & M2 & $\mathrm{H}_{2} \mathrm{O}$ & $\mathrm{N}$ & $\mathrm{N}$ & $\mathrm{N}$ & + & $\mathrm{N}$ & $\mathrm{N}$ & $\mathrm{N}$ & $\mathrm{N}$ & $\mathrm{N}$ & $\mathrm{N}$ & $\mathrm{N}$ & $\mathrm{N}$ & $\mathrm{N}$ & $\mathrm{N}$ & $\mathrm{N}$ & $\mathrm{N}$ & $\mathrm{N}$ & $\mathrm{N}$ & $\mathrm{N}$ & $\mathrm{N}$ & $\begin{array}{l}\text { Abd El Nabi } \\
\text { et al. } 1992\end{array}$ \\
\hline & & & \multirow{8}{*}{ Se. } & \multirow{4}{*}{ M3 } & $\mathrm{MeOH}$ & + & $\mathrm{N}$ & $\mathrm{N}$ & $\mathrm{N}$ & $\mathrm{N}$ & $\mathrm{N}$ & $\mathrm{N}$ & + & $\mathrm{N}$ & $\mathrm{N}$ & $\mathrm{N}$ & $\mathrm{N}$ & $\mathrm{N}$ & $\mathrm{N}$ & $\mathrm{N}$ & $\mathrm{N}$ & $\mathrm{N}$ & $\mathrm{N}$ & $\mathrm{N}$ & $\mathrm{N}$ & \multirow{22}{*}{$\begin{array}{l}\text { Abdel- } \\
\text { Rahim et al. } \\
\text { 2012c }\end{array}$} \\
\hline & & & & & di.ether & + & $\mathrm{N}$ & $\mathrm{N}$ & $\mathrm{N}$ & $\mathrm{N}$ & $\mathrm{N}$ & $\mathrm{N}$ & + & $\mathrm{N}$ & $\mathrm{N}$ & $\mathrm{N}$ & $\mathrm{N}$ & $\mathrm{N}$ & $\mathrm{N}$ & $\mathrm{N}$ & $\mathrm{N}$ & $\mathrm{N}$ & $\mathrm{N}$ & $\mathrm{N}$ & $\mathrm{N}$ & \\
\hline & & & & & Hex. & + & $\mathrm{N}$ & $\mathrm{N}$ & $\mathrm{N}$ & $\mathrm{N}$ & $\mathrm{N}$ & $\mathrm{N}$ & + & $\mathrm{N}$ & $\mathrm{N}$ & $\mathrm{N}$ & $\mathrm{N}$ & $\mathrm{N}$ & $\mathrm{N}$ & $\mathrm{N}$ & $\mathrm{N}$ & $\mathrm{N}$ & $\mathrm{N}$ & $\mathrm{N}$ & $\mathrm{N}$ & \\
\hline & & & & & $\mathrm{CHCl}_{3}$ & + & $\mathrm{N}$ & $\mathrm{N}$ & $\mathrm{N}$ & $\mathrm{N}$ & $\mathrm{N}$ & $\mathrm{N}$ & + & $\mathrm{N}$ & $\mathrm{N}$ & $\mathrm{N}$ & $\mathrm{N}$ & $\mathrm{N}$ & $\mathrm{N}$ & $\mathrm{N}$ & $\mathrm{N}$ & $\mathrm{N}$ & $\mathrm{N}$ & $\mathrm{N}$ & $\mathrm{N}$ & \\
\hline & & & & \multirow{4}{*}{ M3 } & EtOH & + & $\mathrm{N}$ & $\mathrm{N}$ & $\mathrm{N}$ & $\mathrm{N}$ & $\mathrm{N}$ & $\mathrm{N}$ & + & $\mathrm{N}$ & $\mathrm{N}$ & $\mathrm{N}$ & $\mathrm{N}$ & $\mathrm{N}$ & $\mathrm{N}$ & $\mathrm{N}$ & $\mathrm{N}$ & $\mathrm{N}$ & $\mathrm{N}$ & $\mathrm{N}$ & $\mathrm{N}$ & \\
\hline & & & & & Acet. & + & $\mathrm{N}$ & $\mathrm{N}$ & $\mathrm{N}$ & $\mathrm{N}$ & $\mathrm{N}$ & $\mathrm{N}$ & + & $\mathrm{N}$ & $\mathrm{N}$ & $\mathrm{N}$ & $\mathrm{N}$ & $\mathrm{N}$ & $\mathrm{N}$ & $\mathrm{N}$ & $\mathrm{N}$ & $\mathrm{N}$ & $\mathrm{N}$ & $\mathrm{N}$ & $\mathrm{N}$ & \\
\hline & & & & & $\begin{array}{l}\text { Pet. } \\
\text { ether }\end{array}$ & + & $\mathrm{N}$ & $\mathrm{N}$ & $\mathrm{N}$ & $\mathrm{N}$ & $\mathrm{N}$ & $\mathrm{N}$ & + & $\mathrm{N}$ & $\mathrm{N}$ & $\mathrm{N}$ & $\mathrm{N}$ & $\mathrm{N}$ & $\mathrm{N}$ & $\mathrm{N}$ & $\mathrm{N}$ & $\mathrm{N}$ & $\mathrm{N}$ & $\mathrm{N}$ & $\mathrm{N}$ & \\
\hline & & & & & $\mathrm{H}_{2} \mathrm{O}$ & + & $\mathrm{N}$ & $\mathrm{N}$ & $\mathrm{N}$ & $\mathrm{N}$ & $\mathrm{N}$ & $\mathrm{N}$ & + & $\mathrm{N}$ & $\mathrm{N}$ & $\mathrm{N}$ & $\mathrm{N}$ & $\mathrm{N}$ & $\mathrm{N}$ & $\mathrm{N}$ & $\mathrm{N}$ & $\mathrm{N}$ & $\mathrm{N}$ & $\mathrm{N}$ & $\mathrm{N}$ & \\
\hline & & & \multirow{7}{*}{ Le. } & \multirow{7}{*}{ M3 } & $\mathrm{MeOH}$ & + & $\mathrm{N}$ & $\mathrm{N}$ & $\mathrm{N}$ & $\mathrm{N}$ & $\mathrm{N}$ & $\mathrm{N}$ & + & $\mathrm{N}$ & $\mathrm{N}$ & $\mathrm{N}$ & $\mathrm{N}$ & $\mathrm{N}$ & $\mathrm{N}$ & $\mathrm{N}$ & $\mathrm{N}$ & $\mathrm{N}$ & $\mathrm{N}$ & $\mathrm{N}$ & $\mathrm{N}$ & \\
\hline & & & & & di.ether & + & $\mathrm{N}$ & $\mathrm{N}$ & $\mathrm{N}$ & $\mathrm{N}$ & $\mathrm{N}$ & $\mathrm{N}$ & + & $\mathrm{N}$ & $\mathrm{N}$ & $\mathrm{N}$ & $\mathrm{N}$ & $\mathrm{N}$ & $\mathrm{N}$ & $\mathrm{N}$ & $\mathrm{N}$ & $\mathrm{N}$ & $\mathrm{N}$ & $\mathrm{N}$ & $\mathrm{N}$ & \\
\hline & & & & & Hex. & + & $\mathrm{N}$ & $\mathrm{N}$ & $\mathrm{N}$ & $\mathrm{N}$ & $\mathrm{N}$ & $\mathrm{N}$ & + & $\mathrm{N}$ & $\mathrm{N}$ & $\mathrm{N}$ & $\mathrm{N}$ & $\mathrm{N}$ & $\mathrm{N}$ & $\mathrm{N}$ & $\mathrm{N}$ & $\mathrm{N}$ & $\mathrm{N}$ & $\mathrm{N}$ & $\mathrm{N}$ & \\
\hline & & & & & $\mathrm{CHCl}_{3}$ & + & $\mathrm{N}$ & $\mathrm{N}$ & $\mathrm{N}$ & $\mathrm{N}$ & $\mathrm{N}$ & $\mathrm{N}$ & + & $\mathrm{N}$ & $\mathrm{N}$ & $\mathrm{N}$ & $\mathrm{N}$ & $\mathrm{N}$ & $\mathrm{N}$ & $\mathrm{N}$ & $\mathrm{N}$ & $\mathrm{N}$ & $\mathrm{N}$ & $\mathrm{N}$ & $\mathrm{N}$ & \\
\hline & & & & & EtOH & + & $\mathrm{N}$ & $\mathrm{N}$ & $\mathrm{N}$ & $\mathrm{N}$ & $\mathrm{N}$ & $\mathrm{N}$ & + & $\mathrm{N}$ & $\mathrm{N}$ & $\mathrm{N}$ & $\mathrm{N}$ & $\mathrm{N}$ & $\mathrm{N}$ & $\mathrm{N}$ & $\mathrm{N}$ & $\mathrm{N}$ & $\mathrm{N}$ & $\mathrm{N}$ & $\mathrm{N}$ & \\
\hline & & & & & Acet. & + & $\mathrm{N}$ & $\mathrm{N}$ & $\mathrm{N}$ & $\mathrm{N}$ & $\mathrm{N}$ & $\mathrm{N}$ & + & $\mathrm{N}$ & $\mathrm{N}$ & $\mathrm{N}$ & $\mathrm{N}$ & $\mathrm{N}$ & $\mathrm{N}$ & $\mathrm{N}$ & $\mathrm{N}$ & $\mathrm{N}$ & $\mathrm{N}$ & $\mathrm{N}$ & $\mathrm{N}$ & \\
\hline & & & & & $\begin{array}{l}\text { Pet. } \\
\text { ether }\end{array}$ & + & $\mathrm{N}$ & $\mathrm{N}$ & $\mathrm{N}$ & $\mathrm{N}$ & $\mathrm{N}$ & $\mathrm{N}$ & + & $\mathrm{N}$ & $\mathrm{N}$ & $\mathrm{N}$ & $\mathrm{N}$ & $\mathrm{N}$ & $\mathrm{N}$ & $\mathrm{N}$ & $\mathrm{N}$ & $\mathrm{N}$ & $\mathrm{N}$ & $\mathrm{N}$ & $\mathrm{N}$ & \\
\hline & & & \multirow{7}{*}{ Po. } & \multirow{7}{*}{ M3 } & $\mathrm{H}_{2} \mathrm{O}$ & + & $\mathrm{N}$ & $\mathrm{N}$ & $\mathrm{N}$ & $\mathrm{N}$ & $\mathrm{N}$ & $\mathrm{N}$ & + & $\mathrm{N}$ & $\mathrm{N}$ & $\mathrm{N}$ & $\mathrm{N}$ & $\mathrm{N}$ & $\mathrm{N}$ & $\mathrm{N}$ & $\mathrm{N}$ & $\mathrm{N}$ & $\mathrm{N}$ & $\mathrm{N}$ & $\mathrm{N}$ & \\
\hline & & & & & $\mathrm{MeOH}$ & + & $\mathrm{N}$ & $\mathrm{N}$ & $\mathrm{N}$ & $\mathrm{N}$ & $\mathrm{N}$ & $\mathrm{N}$ & + & $\mathrm{N}$ & $\mathrm{N}$ & $\mathrm{N}$ & $\mathrm{N}$ & $\mathrm{N}$ & $\mathrm{N}$ & $\mathrm{N}$ & $\mathrm{N}$ & $\mathrm{N}$ & $\mathrm{N}$ & $\mathrm{N}$ & $\mathrm{N}$ & \\
\hline & & & & & di.ether & + & $\mathrm{N}$ & $\mathrm{N}$ & $\mathrm{N}$ & $\mathrm{N}$ & $\mathrm{N}$ & $\mathrm{N}$ & + & $\mathrm{N}$ & $\mathrm{N}$ & $\mathrm{N}$ & $\mathrm{N}$ & $\mathrm{N}$ & $\mathrm{N}$ & $\mathrm{N}$ & $\mathrm{N}$ & $\mathrm{N}$ & $\mathrm{N}$ & $\mathrm{N}$ & $\mathrm{N}$ & \\
\hline & & & & & Hex. & + & $\mathrm{N}$ & $\mathrm{N}$ & $\mathrm{N}$ & $\mathrm{N}$ & $\mathrm{N}$ & $\mathrm{N}$ & + & $\mathrm{N}$ & $\mathrm{N}$ & $\mathrm{N}$ & $\mathrm{N}$ & $\mathrm{N}$ & $\mathrm{N}$ & $\mathrm{N}$ & $\mathrm{N}$ & $\mathrm{N}$ & $\mathrm{N}$ & $\mathrm{N}$ & $\mathrm{N}$ & \\
\hline & & & & & $\mathrm{CHCl}_{3}$ & + & $\mathrm{N}$ & $\mathrm{N}$ & $\mathrm{N}$ & $\mathrm{N}$ & $\mathrm{N}$ & $\mathrm{N}$ & + & $\mathrm{N}$ & $\mathrm{N}$ & $\mathrm{N}$ & $\mathrm{N}$ & $\mathrm{N}$ & $\mathrm{N}$ & $\mathrm{N}$ & $\mathrm{N}$ & $\mathrm{N}$ & $\mathrm{N}$ & $\mathrm{N}$ & $\mathrm{N}$ & \\
\hline & & & & & EtOH & + & $\mathrm{N}$ & $\mathrm{N}$ & $\mathrm{N}$ & $\mathrm{N}$ & $\mathrm{N}$ & $\mathrm{N}$ & + & $\mathrm{N}$ & $\mathrm{N}$ & $\mathrm{N}$ & $\mathrm{N}$ & $\mathrm{N}$ & $\mathrm{N}$ & $\mathrm{N}$ & $\mathrm{N}$ & $\mathrm{N}$ & $\mathrm{N}$ & $\mathrm{N}$ & $\mathrm{N}$ & \\
\hline & & & & & Acet. & + & $\mathrm{N}$ & $\mathrm{N}$ & $\mathrm{N}$ & $\mathrm{N}$ & $\mathrm{N}$ & $\mathrm{N}$ & + & $\mathrm{N}$ & $\mathrm{N}$ & $\mathrm{N}$ & $\mathrm{N}$ & $\mathrm{N}$ & $\mathrm{N}$ & $\mathrm{N}$ & $\mathrm{N}$ & $\mathrm{N}$ & $\mathrm{N}$ & $\mathrm{N}$ & $\mathrm{N}$ & \\
\hline
\end{tabular}




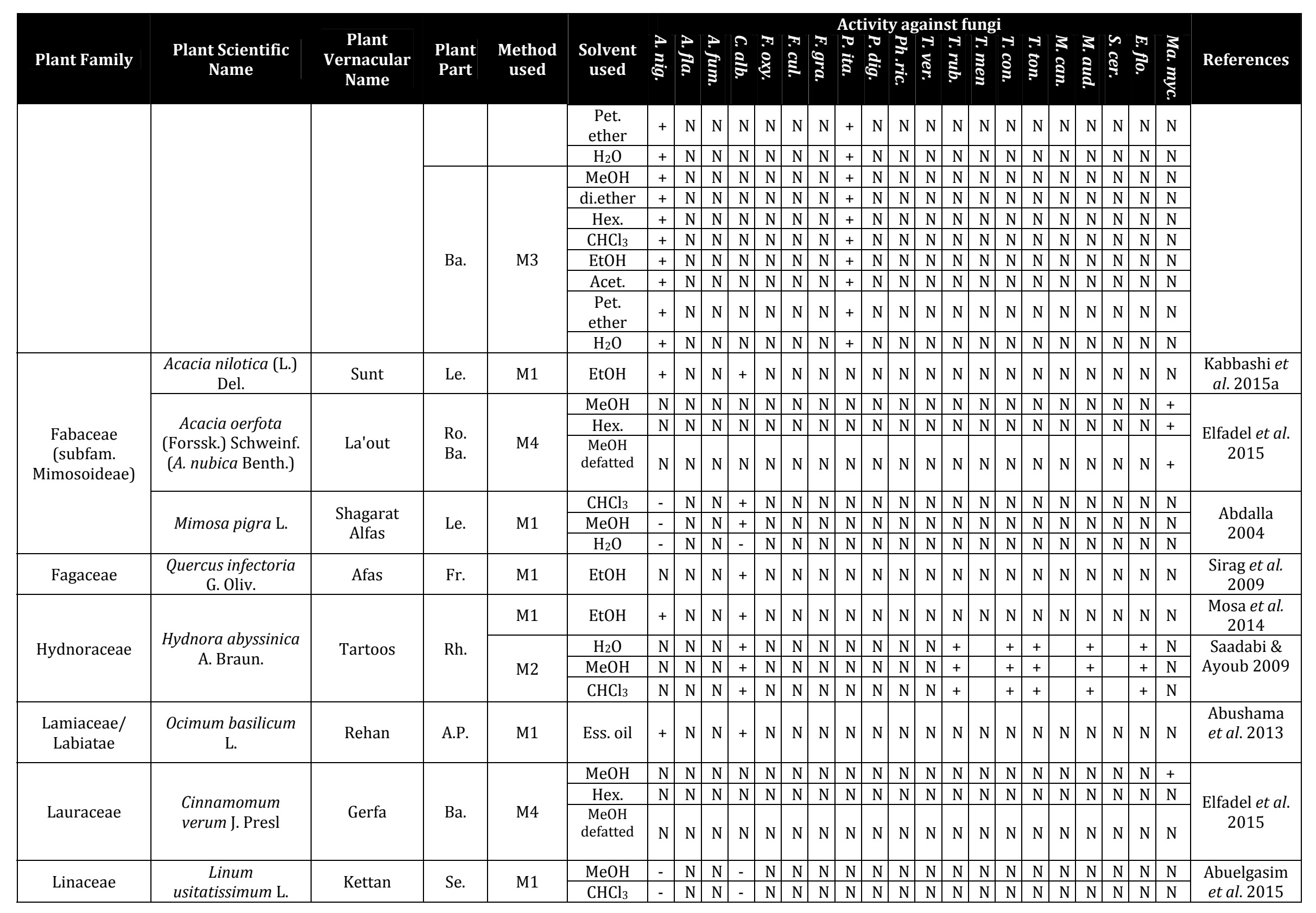




\begin{tabular}{|c|c|c|c|c|c|c|c|c|c|c|c|c|c|c|c|c|c|c|c|c|c|c|c|c|c|c|}
\hline Plant Family & $\begin{array}{l}\text { Plant Scientific } \\
\text { Name }\end{array}$ & $\begin{array}{c}\text { Plant } \\
\text { Vernacular } \\
\text { Name }\end{array}$ & $\begin{array}{l}\text { Plant } \\
\text { Part }\end{array}$ & $\begin{array}{c}\text { Method } \\
\text { used }\end{array}$ & $\begin{array}{c}\text { Solvent } \\
\text { used }\end{array}$ & 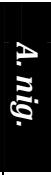 & $\stackrel{?}{\overrightarrow{2}}$ & $\stackrel{p}{3}$ & $\hat{2}$ & in & ב-1 & के & $\begin{array}{l}\text { Act } \\
\vdots \\
\overline{5}\end{array}$ & ivit & 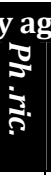 & 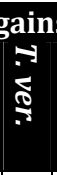 & $\left|\begin{array}{l}1 s t \text { fu } \\
-1 \\
\vdots \\
\vdots \\
\vdots\end{array}\right|$ & 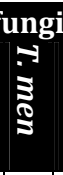 & $\vec{i}$ & $\overrightarrow{\hat{s}}$ & $\mid \begin{array}{l}\mathfrak{S} \\
\mathfrak{s} \\
\mathfrak{S}\end{array}$ & $\begin{array}{l}3 \\
\vdots \\
\vdots \\
\vdots\end{array}$ & $\stackrel{1}{\mathfrak{2}}$ & $\frac{0}{2 \pi}$ & 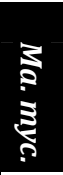 & References \\
\hline & & & & & $\mathrm{H}_{2} \mathrm{O}$ & - & $\mathrm{N}$ & $\mathrm{N}$ & + & $\mathrm{N}$ & $\mathrm{N}$ & $\mathrm{N}$ & $\mathrm{N}$ & $\mathrm{N}$ & $\mathrm{N}$ & $\mathrm{N}$ & $\mathrm{N}$ & $\mathrm{N}$ & $\mathrm{N}$ & $\mathrm{N}$ & $\mathrm{N}$ & $\mathrm{N}$ & $\mathrm{N}$ & \begin{tabular}{|l|}
$\mathrm{N}$ \\
\end{tabular} & $\mathrm{N}$ & \\
\hline \multirow{6}{*}{ Loranthaceae } & \multirow{6}{*}{$\begin{array}{c}\text { Plicosepalus } \\
\text { acaciae (Zucc.) } \\
\text { Weins. et Polhill. }\end{array}$} & \multirow{6}{*}{ Anab El Talh } & \multirow{3}{*}{ Le. } & \multirow{3}{*}{ M1 } & $\mathrm{CHCl}_{3}$ & $\mathrm{~N}$ & $\mathrm{~N}$ & $\mathrm{~N}$ & - & $\mathrm{N}$ & $\mathrm{N}$ & $\mathrm{N}$ & $\mathrm{N}$ & $\mathrm{N}$ & $\mathrm{N}$ & $\mathrm{N}$ & $\mathrm{N}$ & $\mathrm{N}$ & $\mathrm{N}$ & $\mathrm{N}$ & $\mathrm{N}$ & $\mathrm{N}$ & + & $\mathrm{N}$ & $\mathrm{N}$ & \multirow{6}{*}{$\begin{array}{l}\text { Elegami et } \\
\text { al. } 2001\end{array}$} \\
\hline & & & & & $\mathrm{MeOH}$ & $\mathrm{N}$ & $\mathrm{N}$ & $\mathrm{N}$ & + & $\mathrm{N}$ & $\mathrm{N}$ & $\mathrm{N}$ & $\mathrm{N}$ & $\mathrm{N}$ & $\mathrm{N}$ & $\mathrm{N}$ & $\mathrm{N}$ & $\mathrm{N}$ & $\mathrm{N}$ & $\mathrm{N}$ & $\mathrm{N}$ & $\mathrm{N}$ & + & $\mathrm{N}$ & $\mathrm{N}$ & \\
\hline & & & & & $\mathrm{H}_{2} \mathrm{O}$ & $\mathrm{N}$ & $\mathrm{N}$ & $\mathrm{N}$ & \begin{tabular}{|l|}
+ \\
\end{tabular} & $\mathrm{N}$ & $\mathrm{N}$ & $\mathrm{N}$ & $\mathrm{N}$ & $\mathrm{N}$ & $\mathrm{N}$ & $\mathrm{N}$ & $\mathrm{N}$ & $\mathrm{N}$ & $\mathrm{N}$ & $\mathrm{N}$ & $\mathrm{N}$ & $\mathrm{N}$ & + & $\mathrm{N}$ & $\mathrm{N}$ & \\
\hline & & & \multirow{3}{*}{ St. } & \multirow{3}{*}{ M1 } & $\mathrm{CHCl}_{3}$ & $\mathrm{~N}$ & $\mathrm{~N}$ & $\mathrm{~N}$ & - & $\mathrm{N}$ & $\mathrm{N}$ & $\mathrm{N}$ & $\mathrm{N}$ & $\mathrm{N}$ & $\mathrm{N}$ & $\mathrm{N}$ & $\mathrm{N}$ & $\mathrm{N}$ & $\mathrm{N}$ & $\mathrm{N}$ & $\mathrm{N}$ & $\mathrm{N}$ & - & $\mathrm{N}$ & $\mathrm{N}$ & \\
\hline & & & & & $\mathrm{MeOH}$ & $\mathrm{N}$ & $\mathrm{N}$ & $\mathrm{N}$ & + & $\mathrm{N}$ & $\mathrm{N}$ & $\mathrm{N}$ & $\mathrm{N}$ & $\mathrm{N}$ & $\mathrm{N}$ & $\mathrm{N}$ & $\mathrm{N}$ & $\mathrm{N}$ & $\mathrm{N}$ & $\mathrm{N}$ & $\mathrm{N}$ & $\mathrm{N}$ & + & \begin{tabular}{|l|}
$\mathrm{N}$ \\
\end{tabular} & $\mathrm{N}$ & \\
\hline & & & & & $\mathrm{H}_{2} \mathrm{O}$ & $\mathrm{N}$ & $\mathrm{N}$ & $\mathrm{N}$ & + & $\mathrm{N}$ & $\mathrm{N}$ & $\mathrm{N}$ & $\mathrm{N}$ & $\mathrm{N}$ & $\mathrm{N}$ & $\mathrm{N}$ & $\mathrm{N}$ & $\mathrm{N}$ & $\mathrm{N}$ & $\mathrm{N}$ & $\mathrm{N}$ & $\mathrm{N}$ & - & \begin{tabular}{|l|}
$\mathrm{N}$ \\
\end{tabular} & $\mathrm{N}$ & \\
\hline \multirow{6}{*}{ Lythraceae } & \multirow{5}{*}{$\begin{array}{l}\text { Lawsonia inermis } \\
\text { L. }\end{array}$} & \multirow{5}{*}{ Henna' } & \multirow{5}{*}{ Le. } & \multirow{3}{*}{ M1 } & $\mathrm{CHCl}_{3}$ & $\mathrm{~N}$ & $\mathrm{~N}$ & $\mathrm{~N}$ & + & $\mathrm{N}$ & $\mathrm{N}$ & $\mathrm{N}$ & $\mathrm{N}$ & $\mathrm{N}$ & $\mathrm{N}$ & $\mathrm{N}$ & $\mathrm{N}$ & $\mathrm{N}$ & $\mathrm{N}$ & $\mathrm{N}$ & $\mathrm{N}$ & $\mathrm{N}$ & + & $\mathrm{N}$ & $\mathrm{N}$ & \multirow{3}{*}{$\begin{array}{l}\text { Elegami et } \\
\text { al. } 2006\end{array}$} \\
\hline & & & & & $\mathrm{MeOH}$ & $\mathrm{N}$ & $\mathrm{N}$ & $\mathrm{N}$ & + & $\mathrm{N}$ & $\mathrm{N}$ & $\mathrm{N}$ & $\mathrm{N}$ & $\mathrm{N}$ & $\mathrm{N}$ & $\mathrm{N}$ & $\mathrm{N}$ & $\mathrm{N}$ & $\mathrm{N}$ & $\mathrm{N}$ & $\mathrm{N}$ & $\mathrm{N}$ & + & \begin{tabular}{|l|}
$\mathrm{N}$ \\
\end{tabular} & $\mathrm{N}$ & \\
\hline & & & & & $\mathrm{H}_{2} \mathrm{O}$ & $\mathrm{N}$ & $\mathrm{N}$ & $\mathrm{N}$ & + & $\mathrm{N}$ & $\mathrm{N}$ & $\mathrm{N}$ & $\mathrm{N}$ & $\mathrm{N}$ & $\mathrm{N}$ & $\mathrm{N}$ & $\mathrm{N}$ & $\mathrm{N}$ & $\mathrm{N}$ & $\mathrm{N}$ & $\mathrm{N}$ & $\mathrm{N}$ & + & $\mathrm{N}$ & $\mathrm{N}$ & \\
\hline & & & & \multirow{2}{*}{ M2 } & $\mathrm{H}_{2} \mathrm{O}$ & $\mathrm{N}$ & $\mathrm{N}$ & $\mathrm{N}$ & + & $\mathrm{N}$ & $\mathrm{N}$ & $\mathrm{N}$ & $\mathrm{N}$ & $\mathrm{N}$ & $\mathrm{N}$ & $\mathrm{N}$ & + & $\mathrm{N}$ & + & + & $\mathrm{N}$ & + & $\mathrm{N}$ & + & $\mathrm{N}$ & \multirow{2}{*}{$\begin{array}{c}\text { Saadabi } \\
2007\end{array}$} \\
\hline & & & & & $\mathrm{MeOH}$ & $\mathrm{N}$ & $\mathrm{N}$ & $\mathrm{N}$ & + & $\mathrm{N}$ & $\mathrm{N}$ & $\mathrm{N}$ & $\mathrm{N}$ & $\mathrm{N}$ & $\mathrm{N}$ & $\mathrm{N}$ & + & $\mathrm{N}$ & + & + & $\mathrm{N}$ & + & $\mathrm{N}$ & + & $\mathrm{N}$ & \\
\hline & Punica granatum L. & Rumman & Fr.Pe. & M3 & $\mathrm{H}_{2} \mathrm{O}$ & + & $\mathrm{N}$ & $\mathrm{N}$ & $\mathrm{N}$ & $\mathrm{N}$ & $\mathrm{N}$ & $\mathrm{N}$ & + & $\mathrm{N}$ & $\mathrm{N}$ & $\mathrm{N}$ & $\mathrm{N}$ & $\mathrm{N}$ & $\mathrm{N}$ & $\mathrm{N}$ & $\mathrm{N}$ & $\mathrm{N}$ & $\mathrm{N}$ & $\mathrm{N}$ & $\mathrm{N}$ & $\begin{array}{c}\text { Abdel- } \\
\text { Rahim et al. } \\
\text { 2012a }\end{array}$ \\
\hline \multirow{11}{*}{ Malvaceae } & \multirow{3}{*}{$\begin{array}{l}\text { Abutilon pannosum } \\
\text { (G.Forst.) Schltdl. }\end{array}$} & \multirow{3}{*}{ Umbarow } & & & $\mathrm{CHCl}_{3}$ & $\mathrm{~N}$ & $\mathrm{~N}$ & $\mathrm{~N}$ & - & $\mathrm{N}$ & $\mathrm{N}$ & $\mathrm{N}$ & $\mathrm{N}$ & $\mathrm{N}$ & $\mathrm{N}$ & $\mathrm{N}$ & $\mathrm{N}$ & $\mathrm{N}$ & $\mathrm{N}$ & $\mathrm{N}$ & $\mathrm{N}$ & $\mathrm{N}$ & + & $\mathrm{N}$ & $\mathrm{N}$ & \\
\hline & & & W.P. & M1 & $\mathrm{MeOH}$ & $\mathrm{N}$ & $\mathrm{N}$ & $\mathrm{N}$ & + & $\mathrm{N}$ & $\mathrm{N}$ & $\mathrm{N}$ & $\mathrm{N}$ & $\mathrm{N}$ & $\mathrm{N}$ & $\mathrm{N}$ & $\mathrm{N}$ & $\mathrm{N}$ & $\mathrm{N}$ & $\mathrm{N}$ & $\mathrm{N}$ & $\mathrm{N}$ & + & \begin{tabular}{|l|}
$\mathrm{N}$ \\
\end{tabular} & $\mathrm{N}$ & $\begin{array}{l}\text { Elegami et } \\
\text { al. } 2006\end{array}$ \\
\hline & & & & & $\mathrm{H}_{2} \mathrm{O}$ & $\mathrm{N}$ & $\mathrm{N}$ & $\mathrm{N}$ & - & $\mathrm{N}$ & $\mathrm{N}$ & $\mathrm{N}$ & $\mathrm{N}$ & $\mathrm{N}$ & $\mathrm{N}$ & $\mathrm{N}$ & $\mathrm{N}$ & $\mathrm{N}$ & $\mathrm{N}$ & $\mathrm{N}$ & $\mathrm{N}$ & $\mathrm{N}$ & - & $\mathrm{N}$ & $\mathrm{N}$ & \\
\hline & Hibiscus sabdariffa & Karkadeh & Le. & M1 & $\mathrm{MeOH}$ & + & $\mathrm{N}$ & $\mathrm{N}$ & + & $\mathrm{N}$ & $\mathrm{N}$ & $\mathrm{N}$ & $\mathrm{N}$ & $\mathrm{N}$ & $\mathrm{N}$ & $\mathrm{N}$ & $\mathrm{N}$ & $\mathrm{N}$ & $\mathrm{N}$ & $\mathrm{N}$ & $\mathrm{N}$ & $\mathrm{N}$ & $\mathrm{N}$ & $\mathrm{N}$ & $\mathrm{N}$ & Abd-Ulgadir \\
\hline & L. & Karkaden & Se. & M1 & $\mathrm{MeOH}$ & - & $\mathrm{N}$ & $\mathrm{N}$ & - & $\mathrm{N}$ & $\mathrm{N}$ & $\mathrm{N}$ & $\mathrm{N}$ & $\mathrm{N}$ & $\mathrm{N}$ & $\mathrm{N}$ & $\mathrm{N}$ & $\mathrm{N}$ & $\mathrm{N}$ & $\mathrm{N}$ & $\mathrm{N}$ & $\mathrm{N}$ & $\mathrm{N}$ & $\mathrm{N}$ & $\mathrm{N}$ & et al. 2015 \\
\hline & & & & & $\mathrm{CHCl}_{3}$ & $\mathrm{~N}$ & $\mathrm{~N}$ & $\mathrm{~N}$ & - & $\mathrm{N}$ & $\mathrm{N}$ & $\mathrm{N}$ & $\mathrm{N}$ & $\mathrm{N}$ & $\mathrm{N}$ & $\mathrm{N}$ & $\mathrm{N}$ & $\mathrm{N}$ & $\mathrm{N}$ & $\mathrm{N}$ & $\mathrm{N}$ & $\mathrm{N}$ & - & $\mathrm{N}$ & $\mathrm{N}$ & \\
\hline & Hibiscus vitifolius L. & Hambouk & W.P. & M1 & $\mathrm{MeOH}$ & $\mathrm{N}$ & $\mathrm{N}$ & $\mathrm{N}$ & + & $\mathrm{N}$ & $\mathrm{N}$ & $\mathrm{N}$ & $\mathrm{N}$ & $\mathrm{N}$ & $\mathrm{N}$ & $\mathrm{N}$ & $\mathrm{N}$ & $\mathrm{N}$ & $\mathrm{N}$ & $\mathrm{N}$ & $\mathrm{N}$ & \begin{tabular}{l|l}
$\mathrm{N}$ \\
\end{tabular} & + & $\mathrm{N}$ & $\mathrm{N}$ & \\
\hline & & & & & $\mathrm{H}_{2} \mathrm{O}$ & $\mathrm{N}$ & $\mathrm{N}$ & $\mathrm{N}$ & + & $\mathrm{N}$ & $\mathrm{N}$ & $\mathrm{N}$ & $\mathrm{N}$ & $\mathrm{N}$ & $\mathrm{N}$ & $\mathrm{N}$ & $\mathrm{N}$ & $\mathrm{N}$ & $\mathrm{N}$ & $\mathrm{N}$ & $\mathrm{N}$ & $\mathrm{N}$ & - & $\mathrm{N}$ & $\mathrm{N}$ & Elegami et \\
\hline & & & & & $\mathrm{CHCl}_{3}$ & $\mathrm{~N}$ & $\mathrm{~N}$ & $\mathrm{~N}$ & - & $\mathrm{N}$ & $\mathrm{N}$ & $\mathrm{N}$ & $\mathrm{N}$ & $\mathrm{N}$ & $\mathrm{N}$ & $\mathrm{N}$ & $\mathrm{N}$ & $\mathrm{N}$ & $\mathrm{N}$ & $\mathrm{N}$ & $\mathrm{N}$ & $\mathrm{N}$ & - & $\mathrm{N}$ & $\mathrm{N}$ & al. 2006 \\
\hline & Waltheria indica L. & Irg El Nal & W.P. & M1 & $\mathrm{MeOH}$ & $\mathrm{N}$ & $\mathrm{N}$ & $\mathrm{N}$ & + & $\mathrm{N}$ & $\mathrm{N}$ & $\mathrm{N}$ & $\mathrm{N}$ & $\mathrm{N}$ & $\mathrm{N}$ & $\mathrm{N}$ & $\mathrm{N}$ & $\mathrm{N}$ & $\mathrm{N}$ & $\mathrm{N}$ & $\mathrm{N}$ & $\mathrm{N}$ & + & $\mathrm{N}$ & $\mathrm{N}$ & \\
\hline & & & & & $\mathrm{H}_{2} \mathrm{O}$ & $\mathrm{N}$ & $\mathrm{N}$ & $\mathrm{N}$ & \begin{tabular}{|l|}
- \\
\end{tabular} & $\mathrm{N}$ & $\mathrm{N}$ & $\mathrm{N}$ & $\mathrm{N}$ & $\mathrm{N}$ & $\mathrm{N}$ & $\mathrm{N}$ & $\mathrm{N}$ & $\mathrm{N}$ & $\mathrm{N}$ & $\mathrm{N}$ & $\mathrm{N}$ & $\mathrm{N}$ & - & $\mathrm{N}$ & $\mathrm{N}$ & \\
\hline & & & & & $\mathrm{CHCl}_{3}$ & - & $\mathrm{N}$ & $\mathrm{N}$ & + & $\mathrm{N}$ & $\mathrm{N}$ & $\mathrm{N}$ & $\mathrm{N}$ & $\mathrm{N}$ & $\mathrm{N}$ & $\mathrm{N}$ & $\mathrm{N}$ & $\mathrm{N}$ & $\mathrm{N}$ & $\mathrm{N}$ & $\mathrm{N}$ & $\mathrm{N}$ & $\mathrm{N}$ & $\mathrm{N}$ & $\mathrm{N}$ & \\
\hline $\begin{array}{c}\text { ae } \\
\text { ande }\end{array}$ & $\begin{array}{l}\text { 1nospora bakis } \\
\text { (A.Rich.) Miers }\end{array}$ & Irg Alhagar & Ro. & M1 & $\mathrm{MeOH}$ & - & $\mathrm{N}$ & $\mathrm{N}$ & + & $\mathrm{N}$ & $\mathrm{N}$ & $\mathrm{N}$ & $\mathrm{N}$ & $\mathrm{N}$ & $\mathrm{N}$ & $\mathrm{N}$ & $\mathrm{N}$ & $\mathrm{N}$ & $\mathrm{N}$ & $\mathrm{N}$ & $\mathrm{N}$ & $\mathrm{N}$ & $\mathrm{N}$ & $\mathrm{N}$ & $\mathrm{N}$ & $\begin{array}{l}\text { Abaalla } \\
2004\end{array}$ \\
\hline & & & & & $\mathrm{H}_{2} \mathrm{O}$ & - & $\mathrm{N}$ & $\mathrm{N}$ & \begin{tabular}{|l|}
- \\
\end{tabular} & $\mathrm{N}$ & $\mathrm{N}$ & $\mathrm{N}$ & $\mathrm{N}$ & $\mathrm{N}$ & $\mathrm{N}$ & $\mathrm{N}$ & $\mathrm{N}$ & $\mathrm{N}$ & $\mathrm{N}$ & $\mathrm{N}$ & $\mathrm{N}$ & $\mathrm{N}$ & $\mathrm{N}$ & $\mathrm{N}$ & $\mathrm{N}$ & \\
\hline Moraceae & Ficus vasta Forssk. & Gom'aiz & Le. & M1 & EtOH & + & $\mathrm{N}$ & $\mathrm{N}$ & + & $\mathrm{N}$ & $\mathrm{N}$ & $\mathrm{N}$ & $\mathrm{N}$ & $\mathrm{N}$ & $\mathrm{N}$ & $\mathrm{N}$ & $\mathrm{N}$ & $\mathrm{N}$ & $\mathrm{N}$ & $\mathrm{N}$ & $\mathrm{N}$ & $\mathrm{N}$ & $\mathrm{N}$ & $\mathrm{N}$ & $\mathrm{N}$ & $\begin{array}{l}\text { Mosa et al. } \\
2014\end{array}$ \\
\hline & & & & & $\mathrm{MeOH}$ & - & - & $\mathrm{N}$ & \begin{tabular}{|l|}
+ \\
\end{tabular} & $\mathrm{N}$ & $\mathrm{N}$ & $\mathrm{N}$ & $\mathrm{N}$ & $\mathrm{N}$ & $\mathrm{N}$ & $\mathrm{N}$ & $\mathrm{N}$ & $\mathrm{N}$ & $\mathrm{N}$ & $\mathrm{N}$ & $\mathrm{N}$ & $\mathrm{N}$ & $\mathrm{N}$ & $\mathrm{N}$ & $\mathrm{N}$ & \\
\hline & & & Je & M1 & EtOH & - & + & $\mathrm{N}$ & - & $\mathrm{N}$ & $\mathrm{N}$ & $\mathrm{N}$ & $\mathrm{N}$ & $\mathrm{N}$ & $\mathrm{N}$ & $\mathrm{N}$ & $\mathrm{N}$ & $\mathrm{N}$ & $\mathrm{N}$ & $\mathrm{N}$ & $\mathrm{N}$ & $\mathrm{N}$ & $\mathrm{N}$ & $\mathrm{N}$ & $\mathrm{N}$ & Osman et al. \\
\hline Moringaceae & Moringa oleifera & Al ruwag & Le. & IVI & $\begin{array}{l}\text { Pet. } \\
\text { ether }\end{array}$ & - & - & $\mathrm{N}$ & - & $\mathrm{N}$ & $\mathrm{N}$ & $\mathrm{N}$ & $\mathrm{N}$ & $\mathrm{N}$ & $\mathrm{N}$ & $\mathrm{N}$ & $\mathrm{N}$ & $\mathrm{N}$ & $\mathrm{N}$ & $\mathrm{N}$ & $\mathrm{N}$ & $\mathrm{N}$ & $\mathrm{N}$ & $\mathrm{N}$ & $\mathrm{N}$ & 2015 \\
\hline & & & & & $\mathrm{H}_{2} \mathrm{O}$ & + & $\mathrm{N}$ & $\mathrm{N}$ & + & $\mathrm{N}$ & $\mathrm{N}$ & $\mathrm{N}$ & $\mathrm{N}$ & $\mathrm{N}$ & $\mathrm{N}$ & $\mathrm{N}$ & $\mathrm{N}$ & $\mathrm{N}$ & $\mathrm{N}$ & $\mathrm{N}$ & $\mathrm{N}$ & $\mathrm{N}$ & $\mathrm{N}$ & $\mathrm{N}$ & $\mathrm{N}$ & Saadabi \& \\
\hline & & & Se. & M2 & $\mathrm{MeOH}$ & - & $\mathrm{N}$ & $\mathrm{N}$ & - & $\mathrm{N}$ & $\mathrm{N}$ & $\mathrm{N}$ & $\mathrm{N}$ & $\mathrm{N}$ & $\mathrm{N}$ & $\mathrm{N}$ & $\mathrm{N}$ & $\mathrm{N}$ & $\mathrm{N}$ & $\mathrm{N}$ & $\mathrm{N}$ & $\mathrm{N}$ & $\mathrm{N}$ & $\mathrm{N}$ & $\mathrm{N}$ & $\begin{array}{l}\text { Abu Zaid } \\
2011\end{array}$ \\
\hline
\end{tabular}




\begin{tabular}{|c|c|c|c|c|c|c|c|c|c|c|c|c|c|c|c|c|c|c|c|c|c|c|c|c|c|c|}
\hline Plant Family & $\begin{array}{l}\text { Plant Scientific } \\
\text { Name }\end{array}$ & $\begin{array}{c}\text { Plant } \\
\text { Vernacular } \\
\text { Name }\end{array}$ & $\begin{array}{l}\text { Plant } \\
\text { Part } \\
\end{array}$ & $\begin{array}{c}\text { Method } \\
\text { used }\end{array}$ & $\begin{array}{c}\text { Solvent } \\
\text { used }\end{array}$ & $\stackrel{8}{0}$ & 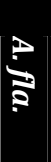 & $\stackrel{p}{5}$ & $\hat{2}$ & $\ddot{x}$ & $\stackrel{-1}{\vdots}$ & $\mid$ & $\begin{array}{l}\text { Acti } \\
: \sigma \\
\tilde{F}\end{array}$ & tivity & 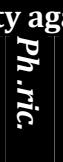 & 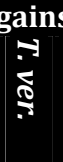 & $\mid \begin{array}{l}3 \\
-1 \\
\vdots \\
\vdots \\
\vdots\end{array}$ & $\mid \begin{array}{l}\text { ungi } \\
-1 \\
3 \\
3 \\
-1\end{array}$ & $\hat{s}$ & $\begin{array}{l}\mathbf{s} \\
\hat{\mathbf{s}}\end{array}$ & 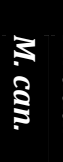 & $\mid \begin{array}{c}\mathbf{s} \\
\vdots \\
\vdots \\
\vdots\end{array}$ & $\stackrel{5}{\$}$ & $\frac{9}{9}$ & $\frac{2}{3}$ & References \\
\hline \multirow{4}{*}{ Myrtaceae } & $\begin{array}{c}\text { Eucalyptus } \\
\text { camaldulensis } \\
\text { Dehn. }\end{array}$ & El Ban & Le. & M1 & Ess. oil & + & $\mathrm{N}$ & $\mathrm{N}$ & + & $\mathrm{N}$ & $\mathrm{N}$ & $\mathrm{N}$ & $\mathrm{N}$ & $\mathrm{N}$ & $\mathrm{N}$ & $\mathrm{N}$ & $\mathrm{N}$ & $\mathrm{N}$ & $\mathrm{N}$ & $\mathrm{N}$ & $\mathrm{N}$ & $\mathrm{N}$ & $\mathrm{N}$ & $\mathrm{N}$ & $\mathrm{N}$ & $\begin{array}{l}\text { Abushama } \\
\text { et al. } 2013\end{array}$ \\
\hline & \multirow{3}{*}{$\begin{array}{c}\text { Syzygium } \\
\text { aromaticum Merr. } \\
\text { \& L.M. Perry } \\
\text { (Eugenia } \\
\text { caryophyllus) } \\
\end{array}$} & \multirow[b]{3}{*}{ Gronful } & \multirow{3}{*}{$\begin{array}{l}\text { Fl. } \\
\text { buds }\end{array}$} & \multirow[b]{3}{*}{ M4 } & $\mathrm{MeOH}$ & $\mathrm{N}$ & $\mathrm{N}$ & $\mathrm{N}$ & $\mathrm{N}$ & $\mathrm{N}$ & $\mathrm{N}$ & $\mathrm{N}$ & $\mathrm{N}$ & $\mathrm{N}$ & $\mathrm{N}$ & $\mathrm{N}$ & $\mathrm{N}$ & $\mathrm{N}$ & $\mathrm{N}$ & $\mathrm{N}$ & $\mathrm{N}$ & $\mathrm{N}$ & $\mathrm{N}$ & $\mathrm{N}$ & + & \multirow{3}{*}{$\begin{array}{l}\text { Elfadel et al } \\
\quad 2015\end{array}$} \\
\hline & & & & & Hex. & $\mathrm{N}$ & $\mathrm{N}$ & $\mathrm{N}$ & $\mathrm{N}$ & $\mathrm{N}$ & $\mathrm{N}$ & $\mathrm{N}$ & $\mathrm{N}$ & \begin{tabular}{|l|}
$\mathrm{N}$ \\
\end{tabular} & $\mathrm{N}$ & $\mathrm{N}$ & $\mathrm{N}$ & $\mathrm{N}$ & \begin{tabular}{|l|}
$\mathrm{N}$ \\
\end{tabular} & $\mathrm{N}$ & $\mathrm{N}$ & $\mathrm{N}$ & $\mathrm{N}$ & $\mathrm{N}$ & $\mathrm{N}$ & \\
\hline & & & & & $\begin{array}{c}\mathrm{MeOH} \\
\text { defatted }\end{array}$ & $\mathrm{N}$ & $\mathrm{N}$ & $\mathrm{N}$ & $\mathrm{N}$ & $\mathrm{N}$ & $\mathrm{N}$ & $\mathrm{N}$ & $\mathrm{N}$ & $\mathrm{N}$ & $\mathrm{N}$ & $\mathrm{N}$ & $\mathrm{N}$ & $\mathrm{N}$ & $\mathrm{N}$ & $\mathrm{N}$ & $\mathrm{N}$ & $\mathrm{N}$ & $\mathrm{N}$ & $\mathrm{N}$ & $\mathrm{N}$ & \\
\hline \multirow{3}{*}{ Nymphaeaceae } & \multirow{3}{*}{ Nymphaea lotus L. } & \multirow{3}{*}{ Suteib } & \multirow{3}{*}{ W.P. } & \multirow{3}{*}{ M1 } & $\mathrm{CHCl}_{3}$ & $\mathrm{~N}$ & $\mathrm{~N}$ & $\mathrm{~N}$ & - & $\mathrm{N}$ & $\mathrm{N}$ & $\mathrm{N}$ & $\mathrm{N}$ & $\mathrm{N}$ & $\mathrm{N}$ & $\mathrm{N}$ & $\mathrm{N}$ & $\mathrm{N}$ & $\mathrm{N}$ & $\mathrm{N}$ & $\mathrm{N}$ & $\mathrm{N}$ & - & $\mathrm{N}$ & $\mathrm{N}$ & \multirow{3}{*}{$\begin{array}{l}\text { El-Egami et } \\
\text { al. } 2005\end{array}$} \\
\hline & & & & & $\mathrm{MeOH}$ & $\mathrm{N}$ & $\mathrm{N}$ & $\mathrm{N}$ & + & $\mathrm{N}$ & $\mathrm{N}$ & $\mathrm{N}$ & $\mathrm{N}$ & $\mathrm{N}$ & $\mathrm{N}$ & $\mathrm{N}$ & $\mathrm{N}$ & $\mathrm{N}$ & $\mathrm{N}$ & $\mathrm{N}$ & $\mathrm{N}$ & $\mathrm{N}$ & + & $\mathrm{N}$ & $\mathrm{N}$ & \\
\hline & & & & & $\mathrm{H}_{2} \mathrm{O}$ & $\mathrm{N}$ & $\mathrm{N}$ & $\mathrm{N}$ & + & $\mathrm{N}$ & $\mathrm{N}$ & $\mathrm{N}$ & $\mathrm{N}$ & $\mathrm{N}$ & $\mathrm{N}$ & $\mathrm{N}$ & $\mathrm{N}$ & $\mathrm{N}$ & $\mathrm{N}$ & $\mathrm{N}$ & $\mathrm{N}$ & $\mathrm{N}$ & + & $\mathrm{N}$ & $\mathrm{N}$ & \\
\hline \multirow{6}{*}{ Olacaceae } & \multirow{6}{*}{$\begin{array}{c}\text { Ximenia americana } \\
\text { L. }\end{array}$} & \multirow{6}{*}{$\begin{array}{l}\text { Um } \\
\text { medeika, } \\
\text { Beu'ok }\end{array}$} & \multirow{3}{*}{ Ba. } & \multirow{3}{*}{ M1 } & $\mathrm{CHCl}_{3}$ & + & $\mathrm{N}$ & $\mathrm{N}$ & + & $\mathrm{N}$ & $\mathrm{N}$ & $\mathrm{N}$ & $\mathrm{N}$ & $\mathrm{N}$ & $\mathrm{N}$ & $\mathrm{N}$ & $\mathrm{N}$ & $\mathrm{N}$ & $\mathrm{N}$ & $\mathrm{N}$ & $\mathrm{N}$ & $\mathrm{N}$ & + & $\mathrm{N}$ & $\mathrm{N}$ & \multirow{6}{*}{$\begin{array}{l}\text { Omer \& } \\
\text { Elnima } \\
2003\end{array}$} \\
\hline & & & & & $\mathrm{MeOH}$ & + & $\mathrm{N}$ & $\mathrm{N}$ & + & $\mathrm{N}$ & $\mathrm{N}$ & $\mathrm{N}$ & $\mathrm{N}$ & $\mathrm{N}$ & $\mathrm{N}$ & $\mathrm{N}$ & $\mathrm{N}$ & $\mathrm{N}$ & $\mathrm{N}$ & $\mathrm{N}$ & $\mathrm{N}$ & $\mathrm{N}$ & + & $\mathrm{N}$ & $\mathrm{N}$ & \\
\hline & & & & & $\mathrm{H}_{2} \mathrm{O}$ & - & $\mathrm{N}$ & $\mathrm{N}$ & + & $\mathrm{N}$ & $\mathrm{N}$ & $\mathrm{N}$ & $\mathrm{N}$ & $\mathrm{N}$ & $\mathrm{N}$ & $\mathrm{N}$ & $\mathrm{N}$ & $\mathrm{N}$ & $\mathrm{N}$ & $\mathrm{N}$ & $\mathrm{N}$ & $\mathrm{N}$ & + & $\mathrm{N}$ & $\mathrm{N}$ & \\
\hline & & & \multirow{3}{*}{ Le. } & \multirow{3}{*}{ M1 } & $\mathrm{CHCl}_{3}$ & + & $\mathrm{N}$ & $\mathrm{N}$ & + & $\mathrm{N}$ & $\mathrm{N}$ & $\mathrm{N}$ & $\mathrm{N}$ & $\mathrm{N}$ & $\mathrm{N}$ & $\mathrm{N}$ & $\mathrm{N}$ & $\mathrm{N}$ & $\mathrm{N}$ & $\mathrm{N}$ & $\mathrm{N}$ & $\mathrm{N}$ & + & $\mathrm{N}$ & $\mathrm{N}$ & \\
\hline & & & & & $\mathrm{MeOH}$ & + & $\mathrm{N}$ & $\mathrm{N}$ & - & $\mathrm{N}$ & $\mathrm{N}$ & $\mathrm{N}$ & $\mathrm{N}$ & $\mathrm{N}$ & $\mathrm{N}$ & $\mathrm{N}$ & $\mathrm{N}$ & $\mathrm{N}$ & $\mathrm{N}$ & $\mathrm{N}$ & $\mathrm{N}$ & $\mathrm{N}$ & + & $\mathrm{N}$ & $\mathrm{N}$ & \\
\hline & & & & & $\mathrm{H}_{2} \mathrm{O}$ & + & $\mathrm{N}$ & $\mathrm{N}$ & - & $\mathrm{N}$ & $\mathrm{N}$ & $\mathrm{N}$ & $\mathrm{N}$ & $\mathrm{N}$ & $\mathrm{N}$ & $\mathrm{N}$ & $\mathrm{N}$ & $\mathrm{N}$ & $\mathrm{N}$ & $\mathrm{N}$ & $\mathrm{N}$ & $\mathrm{N}$ & + & $\mathrm{N}$ & $\mathrm{N}$ & \\
\hline \multirow{6}{*}{ Olacaceae } & & & & & $\mathrm{CHCl}_{3}$ & + & $\mathrm{N}$ & $\mathrm{N}$ & - & $\mathrm{N}$ & $\mathrm{N}$ & $\mathrm{N}$ & $\mathrm{N}$ & $\mathrm{N}$ & $\mathrm{N}$ & $\mathrm{N}$ & $\mathrm{N}$ & $\mathrm{N}$ & \begin{tabular}{|l|}
$\mathrm{N}$ \\
\end{tabular} & $\mathrm{N}$ & \begin{tabular}{l|l}
$\mathrm{N}$ \\
\end{tabular} & $\mathrm{N}$ & + & $\mathrm{N}$ & $\mathrm{N}$ & \\
\hline & & & Ro. & M1 & $\mathrm{MeOH}$ & + & $\mathrm{N}$ & $\mathrm{N}$ & - & $\mathrm{N}$ & $\mathrm{N}$ & $\mathrm{N}$ & $\mathrm{N}$ & $\mathrm{N}$ & $\mathrm{N}$ & $\mathrm{N}$ & $\mathrm{N}$ & $\mathrm{N}$ & \begin{tabular}{|l|}
$\mathrm{N}$ \\
\end{tabular} & $\mathrm{N}$ & $\mathrm{N}$ & $\mathrm{N}$ & + & $\mathrm{N}$ & $\mathrm{N}$ & \\
\hline & Ximenia americana & & & & $\mathrm{H}_{2} \mathrm{O}$ & + & $\mathrm{N}$ & $\mathrm{N}$ & - & $\mathrm{N}$ & $\mathrm{N}$ & $\mathrm{N}$ & $\mathrm{N}$ & $\mathrm{N}$ & $\mathrm{N}$ & $\mathrm{N}$ & $\mathrm{N}$ & $\mathrm{N}$ & $\mathrm{N}$ & $\mathrm{N}$ & $\mathrm{N}$ & $\mathrm{N}$ & - & $\mathrm{N}$ & $\mathrm{N}$ & $\begin{array}{l}\text { Umer \& } \\
\text { Elnima }\end{array}$ \\
\hline & L. & $\begin{array}{l}\text { medelka, } \\
\text { Beu'ok }\end{array}$ & & & $\mathrm{CHCl}_{3}$ & - & $\mathrm{N}$ & $\mathrm{N}$ & + & $\mathrm{N}$ & $\mathrm{N}$ & $\mathrm{N}$ & $\mathrm{N}$ & $\mathrm{N}$ & $\mathrm{N}$ & $\mathrm{N}$ & $\mathrm{N}$ & $\mathrm{N}$ & \begin{tabular}{|l|}
$\mathrm{N}$ \\
\end{tabular} & \begin{tabular}{|l|}
$\mathrm{N}$ \\
\end{tabular} & $\mathrm{N}$ & $\mathrm{N}$ & + & $\mathrm{N}$ & $\mathrm{N}$ & $\begin{array}{l}\text { Einıma } \\
2003\end{array}$ \\
\hline & & & St. & M1 & $\mathrm{MeOH}$ & + & $\mathrm{N}$ & $\mathrm{N}$ & - & $\mathrm{N}$ & $\mathrm{N}$ & $\mathrm{N}$ & $\mathrm{N}$ & $\mathrm{N}$ & $\mathrm{N}$ & $\mathrm{N}$ & $\mathrm{N}$ & $\mathrm{N}$ & \begin{tabular}{|l|}
$\mathrm{N}$ \\
\end{tabular} & $\mathrm{N}$ & $\mathrm{N}$ & $\mathrm{N}$ & + & $\mathrm{N}$ & $\mathrm{N}$ & \\
\hline & & & & & $\mathrm{H}_{2} \mathrm{O}$ & + & $\mathrm{N}$ & & - & $\mathrm{N}$ & $\mathrm{N}$ & $\mathrm{N}$ & $\mathrm{N}$ & $\mathrm{N}$ & $\mathrm{N}$ & $\mathrm{N}$ & $\mathrm{N}$ & $\mathrm{N}$ & $\mathrm{N}$ & $\mathrm{N}$ & $\mathrm{N}$ & $\mathrm{N}$ & + & $\mathrm{N}$ & $\mathrm{N}$ & \\
\hline & Ludwigia erecta (L. & & & & $\mathrm{CHCl}_{3}$ & $\mathrm{~N}$ & $\mathrm{~N}$ & $\mathrm{~N}$ & \begin{tabular}{|l|}
- \\
\end{tabular} & $\mathrm{N}$ & $\mathrm{N}$ & $\mathrm{N}$ & $\mathrm{N}$ & $\mathrm{N}$ & $\mathrm{N}$ & $\mathrm{N}$ & $\mathrm{N}$ & $\mathrm{N}$ & $\mathrm{N}$ & $\mathrm{N}$ & \begin{tabular}{|l|}
$\mathrm{N}$ \\
\end{tabular} & $\mathrm{N}$ & + & $\mathrm{N}$ & $\mathrm{N}$ & \\
\hline Onagraceae & ) H.Hara Uussiaea & Arkala & W.P. & M1 & $\mathrm{MeOH}$ & $\mathrm{N}$ & $\mathrm{N}$ & $\mathrm{N}$ & + & $\mathrm{N}$ & $\mathrm{N}$ & $\mathrm{N}$ & $\mathrm{N}$ & $\mathrm{N}$ & $\mathrm{N}$ & $\mathrm{N}$ & $\mathrm{N}$ & $\mathrm{N}$ & $\mathrm{N}$ & $\mathrm{N}$ & $\mathrm{N}$ & $\mathrm{N}$ & + & $\mathrm{N}$ & $\mathrm{N}$ & El-Egami et \\
\hline & erecta L.) & & & & $\mathrm{H}_{2} \mathrm{O}$ & $\mathrm{N}$ & $\mathrm{N}$ & $\mathrm{N}$ & + & $\mathrm{N}$ & $\mathrm{N}$ & $\mathrm{N}$ & $\mathrm{N}$ & $\mathrm{N}$ & $\mathrm{N}$ & $\mathrm{N}$ & $\mathrm{N}$ & $\mathrm{N}$ & $\mathrm{N}$ & $\mathrm{N}$ & $\mathrm{N}$ & $\mathrm{N}$ & + & $\mathrm{N}$ & $\mathrm{N}$ & \\
\hline & $\begin{array}{c}\text { Cymbopogon } \\
\text { nervatus Chiov. }\end{array}$ & $\mathrm{Nal}$ & A.P. & M1 & Ess. oil & + & + & $\mathrm{N}$ & + & + & $\mathrm{N}$ & $\mathrm{N}$ & $\mathrm{N}$ & $\mathrm{N}$ & $\mathrm{N}$ & $\mathrm{N}$ & $\mathrm{N}$ & $\mathrm{N}$ & $\mathrm{N}$ & $\mathrm{N}$ & $\mathrm{N}$ & $\mathrm{N}$ & $\mathrm{N}$ & $\mathrm{N}$ & $\mathrm{N}$ & \\
\hline $\begin{array}{l}\text { Poaceae/ } \\
\text { Graminae }\end{array}$ & $\begin{array}{c}\text { Cymbopogon } \\
\text { schoenanthus (L.) } \\
\text { Spreng. ssp. } \\
\text { proximus } \\
\end{array}$ & Mahareib & A.P. & M1 & Ess. oil & + & $\mathrm{N}$ & $\mathrm{N}$ & + & $\mathrm{N}$ & $\mathrm{N}$ & $\mathrm{N}$ & $\mathrm{N}$ & $\mathrm{N}$ & $\mathrm{N}$ & $\mathrm{N}$ & $\mathrm{N}$ & $\mathrm{N}$ & $\mathrm{N}$ & $\mathrm{N}$ & $\mathrm{N}$ & $\mathrm{N}$ & $\mathrm{N}$ & $\mathrm{N}$ & $\mathrm{N}$ & $\begin{array}{l}\text { Abushama } \\
\text { et al. } 2013\end{array}$ \\
\hline & Securidaca & & & & $\mathrm{CHCl}_{3}$ & + & $\mathrm{N}$ & $\mathrm{N}$ & + & $\mathrm{N}$ & $\mathrm{N}$ & $\mathrm{N}$ & $\mathrm{N}$ & $\mathrm{N}$ & $\mathrm{N}$ & $\mathrm{N}$ & $\mathrm{N}$ & $\mathrm{N}$ & $\mathrm{N}$ & $\mathrm{N}$ & $\mathrm{N}$ & $\mathrm{N}$ & $\mathrm{N}$ & $\mathrm{N}$ & $\mathrm{N}$ & \\
\hline Polygalaceae & longepedunculata & Irg Alali & Ro. & M1 & $\mathrm{MeOH}$ & - & $\mathrm{N}$ & $\mathrm{N}$ & - & $\mathrm{N}$ & $\mathrm{N}$ & $\mathrm{N}$ & $\mathrm{N}$ & $\mathrm{N}$ & $\mathrm{N}$ & $\mathrm{N}$ & $\mathrm{N}$ & $\mathrm{N}$ & $\mathrm{N}$ & $\mathrm{N}$ & $\mathrm{N}$ & $\mathrm{N}$ & $\mathrm{N}$ & $\mathrm{N}$ & $\mathrm{N}$ & $\begin{array}{l}\text { Abdalla } \\
2004\end{array}$ \\
\hline & Fres. & & & & $\mathrm{H}_{2} \mathrm{O}$ & - & $\mathrm{N}$ & $\mathrm{N}$ & - & $\mathrm{N}$ & $\mathrm{N}$ & $\mathrm{N}$ & $\mathrm{N}$ & $\mathrm{N}$ & $\mathrm{N}$ & $\mathrm{N}$ & $\mathrm{N}$ & $\mathrm{N}$ & $\mathrm{N}$ & $\mathrm{N}$ & $\mathrm{N}$ & $\mathrm{N}$ & $\mathrm{N}$ & $\mathrm{N}$ & $\mathrm{N}$ & \\
\hline & Piper nigrum L & & & & $\mathrm{MeOH}$ & $\mathrm{N}$ & $\mathrm{N}$ & $\mathrm{N}$ & $\mathrm{N}$ & $\mathrm{N}$ & $\mathrm{N}$ & $\mathrm{N}$ & $\mathrm{N}$ & $\mathrm{N}$ & $\mathrm{N}$ & $\mathrm{N}$ & $\mathrm{N}$ & $\mathrm{N}$ & $\mathrm{N}$ & $\mathrm{N}$ & $\mathrm{N}$ & $\mathrm{N}$ & $\mathrm{N}$ & $\mathrm{N}$ & + & Elfadel et al. \\
\hline$r$ & Ptper nigrum L. & $\mathrm{F}$ & Fr. & IV & Hex. & $\mathrm{N}$ & $\mathrm{N}$ & $\mathrm{N}$ & $\mathrm{N}$ & $\mathrm{N}$ & $\mathrm{N}$ & $\mathrm{N}$ & $\mathrm{N}$ & $\mathrm{N} \mid$ & \begin{tabular}{|l|}
$\mathrm{N}$ \\
\end{tabular} & $\mathrm{N}$ & $\mathrm{N}$ & \begin{tabular}{|l|l|}
$\mathrm{N}$ \\
\end{tabular} & \begin{tabular}{|l|l|}
$\mathrm{N}$ \\
\end{tabular} & \begin{tabular}{|l|}
$\mathrm{N}$ \\
\end{tabular} & \begin{tabular}{|l|}
$\mathrm{N}$ \\
\end{tabular} & \begin{tabular}{|l|l|}
$\mathrm{N}$ \\
\end{tabular} & $\mathrm{N}$ & $\mathrm{N}$ & $\mathrm{N}$ & 2015 \\
\hline
\end{tabular}




\begin{tabular}{|c|c|c|c|c|c|c|c|c|c|c|c|c|c|c|c|c|c|c|c|c|c|c|c|c|c|c|}
\hline \multirow[t]{2}{*}{ Plant Family } & $\begin{array}{c}\text { Plant Scientific } \\
\text { Name }\end{array}$ & $\begin{array}{c}\text { Plant } \\
\text { Vernacular } \\
\text { Name }\end{array}$ & $\begin{array}{l}\text { Plant } \\
\text { Part } \\
\end{array}$ & $\begin{array}{c}\text { Method } \\
\text { used }\end{array}$ & $\begin{array}{c}\text { Solvent } \\
\text { used }\end{array}$ & 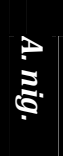 & 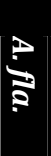 & 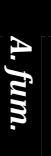 & $\hat{0}$ & $\overrightarrow{0}$ & $\stackrel{-1}{\vdots}$ & के & $\begin{array}{l}\text { Acti } \\
: \sigma \\
\tilde{F}\end{array} \mid$ & tivit & 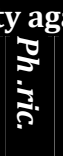 & 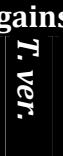 & $\mid \begin{array}{l}1 \\
0 \\
3 \\
3\end{array}$ & 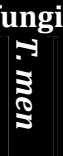 & $\dot{\hat{s}}$ & $\begin{array}{c}\hat{s} \\
\hat{s}\end{array}$ & $\mid \begin{array}{c}\mathfrak{s} \\
\vdots \\
\vdots\end{array}$ & $\mid \begin{array}{c}\mathbf{3} \\
\vdots \\
\vdots \\
\vdots\end{array}$ & $\stackrel{2}{\mathfrak{9}}$ & $\frac{2}{n+\pi}$ & 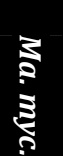 & References \\
\hline & & & & & $\begin{array}{c}\mathrm{MeOH} \\
\text { defatted }\end{array}$ & $\mathrm{N}$ & $\mathrm{N}$ & $\mathrm{N}$ & $\mathrm{N}$ & $\mathrm{N}$ & $\mathrm{N}$ & $\mathrm{N}$ & $\mathrm{N}$ & $\mathrm{N}$ & $\mathrm{N}$ & $\mathrm{N}$ & $\mathrm{N}$ & $\mathrm{N}$ & $\mathrm{N}$ & $\mathrm{N}$ & $\mathrm{N}$ & $\mathrm{N}$ & $\mathrm{N}$ & $\mathrm{N}$ & $\mathrm{N}$ & \\
\hline \multirow{4}{*}{ Ranunculaceae } & \multirow{4}{*}{ Nigella sativa L. } & \multirow{4}{*}{$\begin{array}{l}\text { Habat El } \\
\text { Baraka }\end{array}$} & \multirow{4}{*}{ Se. } & \multirow[b]{3}{*}{ M4 } & $\mathrm{MeOH}$ & $\mathrm{N}$ & $\mathrm{N}$ & $\mathrm{N}$ & $\mathrm{N}$ & $\mathrm{N}$ & $\mathrm{N}$ & $\mathrm{N}$ & $\mathrm{N}$ & $\mathrm{N}$ & $\mathrm{N}$ & $\mathrm{N}$ & $\mathrm{N}$ & $\mathrm{N}$ & $\mathrm{N}$ & $\mathrm{N}$ & $\mathrm{N}$ & $\mathrm{N}$ & $\mathrm{N}$ & $\mathrm{N}$ & + & \\
\hline & & & & & Hex. & $\mathrm{N}$ & $\mathrm{N}$ & $\mathrm{N}$ & $\mathrm{N}$ & $\mathrm{N}$ & $\mathrm{N}$ & $\mathrm{N}$ & $\mathrm{N}$ & $\mathrm{N}$ & $\mathrm{N}$ & $\mathrm{N}$ & $\mathrm{N}$ & $\mathrm{N}$ & $\mathrm{N}$ & $\mathrm{N}$ & $\mathrm{N}$ & $\mathrm{N}$ & $\mathrm{N}$ & \begin{tabular}{|l|}
$\mathrm{N}$ \\
\end{tabular} & + & \\
\hline & & & & & $\begin{array}{c}\mathrm{MeOH} \\
\text { defatted }\end{array}$ & $\mathrm{N}$ & $\mathrm{N}$ & $\mathrm{N}$ & $\mathrm{N}$ & $\mathrm{N}$ & $\mathrm{N}$ & $\mathrm{N}$ & $\mathrm{N}$ & $\mathrm{N}$ & $\mathrm{N}$ & $\mathrm{N}$ & $\mathrm{N}$ & $\mathrm{N}$ & $\mathrm{N}$ & $\mathrm{N}$ & $\mathrm{N}$ & $\mathrm{N}$ & $\mathrm{N}$ & $\mathrm{N}$ & + & \\
\hline & & & & M1 & EtOH & + & $\mathrm{N}$ & $\mathrm{N}$ & + & $\mathrm{N}$ & $\mathrm{N}$ & $\mathrm{N}$ & $\mathrm{N}$ & $\mathrm{N}$ & $\mathrm{N}$ & $\mathrm{N}$ & $\mathrm{N}$ & $\mathrm{N}$ & $\mathrm{N}$ & $\mathrm{N}$ & $\mathrm{N}$ & $\mathrm{N}$ & $\mathrm{N}$ & $\mathrm{N}$ & $\mathrm{N}$ & $\begin{array}{c}\text { Kabbashi } e t \\
\text { al.2015b }\end{array}$ \\
\hline \multirow{3}{*}{ Rubiaceae } & \multirow{3}{*}{$\begin{array}{l}\text { Xeromphis nilotica } \\
\text { (Stapf.) Keay }\end{array}$} & \multirow{3}{*}{$\begin{array}{l}\text { Shagarat } \\
\text { Elmarfien }\end{array}$} & \multirow{3}{*}{ Br. } & \multirow{3}{*}{ M1 } & $\mathrm{CHCl}_{3}$ & + & $\mathrm{N}$ & $\mathrm{N}$ & + & $\mathrm{N}$ & $\mathrm{N}$ & $\mathrm{N}$ & $\mathrm{N}$ & $\mathrm{N}$ & $\mathrm{N}$ & $\mathrm{N}$ & $\mathrm{N}$ & $\mathrm{N}$ & $\mathrm{N}$ & $\mathrm{N}$ & $\mathrm{N}$ & $\mathrm{N}$ & \begin{tabular}{|l|}
$\mathrm{N}$ \\
\end{tabular} & \begin{tabular}{|l|}
$\mathrm{N}$ \\
\end{tabular} & $\mathrm{N}$ & \multirow{3}{*}{$\begin{array}{l}\text { Abdalla } \\
2004\end{array}$} \\
\hline & & & & & $\mathrm{MeOH}$ & - & $\mathrm{N}$ & $\mathrm{N}$ & + & $\mathrm{N}$ & $\mathrm{N}$ & $\mathrm{N}$ & $\mathrm{N}$ & $\mathrm{N}$ & $\mathrm{N}$ & $\mathrm{N}$ & $\mathrm{N}$ & $\mathrm{N}$ & \begin{tabular}{|l|}
$\mathrm{N}$ \\
\end{tabular} & $\mathrm{N}$ & $\mathrm{N}$ & $\mathrm{N}$ & \begin{tabular}{|l|}
$\mathrm{N}$ \\
\end{tabular} & \begin{tabular}{|l|}
$\mathrm{N}$ \\
\end{tabular} & $\mathrm{N}$ & \\
\hline & & & & & $\mathrm{H}_{2} \mathrm{O}$ & - & $\mathrm{N}$ & $\mathrm{N}$ & - & $\mathrm{N}$ & $\mathrm{N}$ & $\mathrm{N}$ & $\mathrm{N}$ & $\mathrm{N}$ & $\mathrm{N}$ & $\mathrm{N}$ & $\mathrm{N}$ & $\mathrm{N}$ & $\mathrm{N}$ & $\mathrm{N}$ & $\mathrm{N}$ & $\mathrm{N}$ & $\mathrm{N}$ & \begin{tabular}{|l|l|}
$\mathrm{N}$ \\
\end{tabular} & $\mathrm{N}$ & \\
\hline Rutaceae & $\begin{array}{l}\text { Citrus paradisi } \\
\text { Macf. }\end{array}$ & Grape Fruit & Fr.Pe. & M1 & Ess. oil & + & $\mathrm{N}$ & $\mathrm{N}$ & + & $\mathrm{N}$ & $\mathrm{N}$ & $\mathrm{N}$ & $\mathrm{N}$ & $\mathrm{N}$ & $\mathrm{N}$ & $\mathrm{N}$ & $\mathrm{N}$ & $\mathrm{N}$ & $\mathrm{N}$ & $\mathrm{N}$ & $\mathrm{N}$ & $\mathrm{N}$ & $\mathrm{N}$ & $\mathrm{N}$ & $\mathrm{N}$ & $\begin{array}{l}\text { Abushama } \\
\text { et al. } 2013\end{array}$ \\
\hline \multirow{6}{*}{ Solanaceae } & \multirow{4}{*}{$\begin{array}{c}\text { Datura } \\
\text { stramonium L. }\end{array}$} & \multirow{4}{*}{ Saikaran } & \multirow[b]{2}{*}{ Le. } & \multirow[b]{2}{*}{ M1 } & $\mathrm{MeOH}$ & - & $\mathrm{N}$ & $\mathrm{N}$ & + & $\mathrm{N}$ & $\mathrm{N}$ & $\mathrm{N}$ & $\mathrm{N}$ & $\mathrm{N}$ & $\mathrm{N}$ & $\mathrm{N}$ & $\mathrm{N}$ & $\mathrm{N}$ & $\mathrm{N}$ & $\mathrm{N}$ & $\mathrm{N}$ & $\mathrm{N}$ & $\mathrm{N}$ & \begin{tabular}{|l|}
$\mathrm{N}$ \\
\end{tabular} & $\mathrm{N}$ & \multirow{4}{*}{$\begin{array}{l}\text { ElNour et al } \\
\quad 2014\end{array}$} \\
\hline & & & & & $\begin{array}{l}\text { Pet. } \\
\text { ether }\end{array}$ & - & $\mathrm{N}$ & $\mathrm{N}$ & - & $\mathrm{N}$ & $\mathrm{N}$ & $\mathrm{N}$ & $\mathrm{N}$ & $\mathrm{N}$ & $\mathrm{N}$ & $\mathrm{N}$ & $\mathrm{N}$ & $\mathrm{N}$ & $\mathrm{N}$ & $\mathrm{N}$ & $\mathrm{N}$ & $\mathrm{N}$ & $\mathrm{N}$ & $\mathrm{N}$ & $\mathrm{N}$ & \\
\hline & & & \multirow[b]{2}{*}{ Le.Ca. } & \multirow[b]{2}{*}{ M1 } & $\mathrm{MeOH}$ & + & $\mathrm{N}$ & $\mathrm{N}$ & - & $\mathrm{N}$ & $\mathrm{N}$ & $\mathrm{N}$ & $\mathrm{N}$ & $\mathrm{N}$ & $\mathrm{N}$ & $\mathrm{N}$ & $\mathrm{N}$ & $\mathrm{N}$ & $\mathrm{N}$ & $\mathrm{N}$ & $\mathrm{N}$ & $\mathrm{N}$ & $\mathrm{N}$ & $\mathrm{N}$ & $\mathrm{N}$ & \\
\hline & & & & & $\begin{array}{l}\text { Pet. } \\
\text { ether }\end{array}$ & - & $\mathrm{N}$ & $\mathrm{N}$ & - & $\mathrm{N}$ & $\mathrm{N}$ & $\mathrm{N}$ & $\mathrm{N}$ & $\mathrm{N}$ & $\mathrm{N}$ & $\mathrm{N}$ & $\mathrm{N}$ & $\mathrm{N}$ & $\mathrm{N}$ & $\mathrm{N}$ & $\mathrm{N}$ & $\mathrm{N}$ & $\mathrm{N}$ & $\mathrm{N}$ & $\mathrm{N}$ & \\
\hline & \multirow{2}{*}{ Solanum nigrum L. } & Tamr el & & & $\mathrm{CHCl}_{3}$ & + & $\mathrm{N}$ & $\mathrm{N}$ & + & $\mathrm{N}$ & $\mathrm{N}$ & $\mathrm{N}$ & $\mathrm{N}$ & $\mathrm{N}$ & $\mathrm{N}$ & $\mathrm{N}$ & $\mathrm{N}$ & $\mathrm{N}$ & $\mathrm{N}$ & $\mathrm{N}$ & $\mathrm{N}$ & $\mathrm{N}$ & $\mathrm{N}$ & $\mathrm{N}$ & $\mathrm{N}$ & Moglad et \\
\hline & & $\begin{array}{c}\text { Abeid, El } \\
\text { Mugt }\end{array}$ & Le. & M1 & $\mathrm{MeOH}$ & + & $\mathrm{N}$ & $\mathrm{N}$ & + & $\mathrm{N}$ & $\mathrm{N}$ & $\mathrm{N}$ & $\mathrm{N}$ & $\mathrm{N}$ & $\mathrm{N}$ & $\mathrm{N}$ & $\mathrm{N}$ & $\mathrm{N}$ & $\mathrm{N}$ & $\mathrm{N}$ & $\mathrm{N}$ & $\mathrm{N}$ & $\mathrm{N}$ & $\mathrm{N}$ & $\mathrm{N}$ & al. 2012 \\
\hline & & & & & $\mathrm{CHCl}_{3}$ & - & $\mathrm{N}$ & $\mathrm{N}$ & - & $\mathrm{N}$ & $\mathrm{N}$ & $\mathrm{N}$ & $\mathrm{N}$ & $\mathrm{N}$ & $\mathrm{N}$ & $\mathrm{N}$ & $\mathrm{N}$ & $\mathrm{N}$ & $\mathrm{N}$ & $\mathrm{N}$ & $\mathrm{N}$ & $\mathrm{N}$ & $\mathrm{N}$ & $\mathrm{N}$ & $\mathrm{N}$ & \\
\hline Tamaricaeae & (L.) Karsten & Tarfa & $\begin{array}{l}\text { Le.t } \\
\text { Br. }\end{array}$ & M1 & $\mathrm{MeOH}$ & + & $\mathrm{N}$ & $\mathrm{N}$ & + & $\mathrm{N}$ & $\mathrm{N}$ & $\mathrm{N}$ & $\mathrm{N}$ & $\mathrm{N}$ & $\mathrm{N}$ & $\mathrm{N}$ & $\mathrm{N}$ & $\mathrm{N}$ & $\mathrm{N}$ & $\mathrm{N}$ & $\mathrm{N}$ & $\mathrm{N}$ & $\mathrm{N}$ & $\mathrm{N}$ & $\mathrm{N}$ & $\begin{array}{l}\text { ADdalla } \\
2004\end{array}$ \\
\hline & & & & & $\mathrm{H}_{2} \mathrm{O}$ & - & $\mathrm{N}$ & $\mathrm{N}$ & - & $\mathrm{N}$ & $\mathrm{N}$ & $\mathrm{N}$ & $\mathrm{N}$ & $\mathrm{N}$ & $\mathrm{N}$ & $\mathrm{N}$ & $\mathrm{N}$ & $\mathrm{N}$ & \begin{tabular}{|l|}
$\mathrm{N}$ \\
\end{tabular} & $\mathrm{N}$ & $\mathrm{N}$ & $\mathrm{N}$ & $\mathrm{N}$ & $\mathrm{N}$ & $\mathrm{N}$ & \\
\hline & Cyphostemma & & & & $\mathrm{CHCl}_{3}$ & $\mathrm{~N}$ & $\mathrm{~N}$ & $\mathrm{~N}$ & + & $\mathrm{N}$ & $\mathrm{N}$ & $\mathrm{N}$ & $\mathrm{N}$ & $\mathrm{N}$ & $\mathrm{N}$ & $\mathrm{N}$ & $\mathrm{N}$ & $\mathrm{N}$ & $\mathrm{N}$ & $\mathrm{N}$ & $\mathrm{N}$ & $\mathrm{N}$ & + & \begin{tabular}{|l|}
$\mathrm{N}$ \\
\end{tabular} & $\mathrm{N}$ & \\
\hline & cyphopetalum & Arnout & W.P. & M1 & $\mathrm{MeOH}$ & $\mathrm{N}$ & $\mathrm{N}$ & $\mathrm{N}$ & + & $\mathrm{N}$ & $\mathrm{N}$ & $\mathrm{N}$ & $\mathrm{N}$ & $\mathrm{N}$ & $\mathrm{N}$ & $\mathrm{N}$ & $\mathrm{N}$ & $\mathrm{N}$ & $\mathrm{N}$ & $\mathrm{N}$ & $\mathrm{N}$ & $\mathrm{N}$ & + & $\mathrm{N}$ & $\mathrm{N}$ & \\
\hline Vitaceae & $\begin{array}{l}\text { (Cissus cyphopetala } \\
\text { Fresen.) }\end{array}$ & & & & $\mathrm{H}_{2} \mathrm{O}$ & $\mathrm{N}$ & $\mathrm{N}$ & $\mathrm{N}$ & - & $\mathrm{N}$ & $\mathrm{N}$ & $\mathrm{N}$ & $\mathrm{N}$ & $\mathrm{N}$ & $\mathrm{N}$ & $\mathrm{N}$ & $\mathrm{N}$ & $\mathrm{N}$ & $\mathrm{N}$ & $\mathrm{N}$ & $\mathrm{N}$ & $\mathrm{N}$ & - & $\mathrm{N}$ & $\mathrm{N}$ & El-Egami et \\
\hline & & & & & $\mathrm{CHCl}_{3}$ & $\mathrm{~N}$ & $\mathrm{~N}$ & $\mathrm{~N}$ & - & $\mathrm{N}$ & $\mathrm{N}$ & $\mathrm{N}$ & $\mathrm{N}$ & $\mathrm{N}$ & $\mathrm{N}$ & $\mathrm{N}$ & $\mathrm{N}$ & $\mathrm{N}$ & $\mathrm{N}$ & $\mathrm{N}$ & $\mathrm{N}$ & $\mathrm{N}$ & - & $\mathrm{N}$ & $\mathrm{N}$ & \\
\hline & quadrangularis L. & Sala'la' & W.P. & M1 & $\mathrm{MeOH}$ & $\mathrm{N}$ & $\mathrm{N}$ & $\mathrm{N}$ & + & $\mathrm{N}$ & $\mathrm{N}$ & $\mathrm{N}$ & $\mathrm{N}$ & $\mathrm{N}$ & $\mathrm{N}$ & $\mathrm{N}$ & $\mathrm{N}$ & $\begin{array}{ll}\mathrm{N} \\
\end{array}$ & \begin{tabular}{|l|}
$\mathrm{N}$ \\
\end{tabular} & $\mathrm{N}$ & $\mathrm{N}$ & $\mathrm{N}$ & + & $\mathrm{N}$ & $\mathrm{N}$ & \\
\hline & & & & & $\mathrm{H}_{2} \mathrm{O}$ & $\mathrm{N}$ & $\mathrm{N}$ & $\mathrm{N}$ & + & $\mathrm{N}$ & $\mathrm{N}$ & $\mathrm{N}$ & $\mathrm{N}$ & $\mathrm{N}$ & $\mathrm{N}$ & $\mathrm{N}$ & $\mathrm{N}$ & $\mathrm{N}$ & \begin{tabular}{|l|}
$\mathrm{N}$ \\
\end{tabular} & $\mathrm{N}$ & \begin{tabular}{|l|}
$\mathrm{N}$ \\
\end{tabular} & \begin{tabular}{|l|}
$\mathrm{N}$ \\
\end{tabular} & - & \begin{tabular}{|l|}
$\mathrm{N}$ \\
\end{tabular} & $\mathrm{N}$ & \\
\hline & & & & & $\mathrm{CHCl}_{3}$ & + & + & & + & $\mathrm{N}$ & $\mathrm{N}$ & $\mathrm{N}$ & $\mathrm{N}$ & $\mathrm{N}$ & + & $\mid \mathrm{N}$ & \begin{tabular}{|l|}
$\mathrm{N}$ \\
\end{tabular} & + & \begin{tabular}{|l|}
$\mathrm{N}$ \\
\end{tabular} & \begin{tabular}{|l|}
$\mathrm{N}$ \\
\end{tabular} & $\mathrm{N}$ & $\mathrm{N}$ & \begin{tabular}{|l|}
$\mathrm{N}$ \\
\end{tabular} & $\mathrm{N}$ & $\mathrm{N}$ & \\
\hline $\begin{array}{l}\text { Xanthorrnoeac } \\
\text { eae (Liliaceae) }\end{array}$ & Aloe sinkatana Rey. & Sabbar & Le. & M1 & $\mathrm{MeOH}$ & + & + & & + & $\mathrm{N}$ & $\mathrm{N}$ & $\mathrm{N}$ & $\mathrm{N}$ & $\mathrm{N}$ & + & $\mathrm{N}$ & $\mathrm{N}$ & + & $\mathrm{N}$ & $\mathrm{N}$ & \begin{tabular}{|l|}
$\mathrm{N}$ \\
\end{tabular} & $\mathrm{N}$ & $\mathrm{N}$ & $\mathrm{N}$ & $\mathrm{N}$ & $\begin{array}{l}\text { All et al. } \\
2013\end{array}$ \\
\hline & & & & & $\mathrm{H}_{2} \mathrm{O}$ & + & - & & - & $\mathrm{N}$ & $\mathrm{N}$ & $\mathrm{N}$ & $\mathrm{N}$ & $\mathrm{N}$ & - & $\mathrm{N}$ & $\mathrm{N}$ & - & $\mathrm{N}$ & $\mathrm{N}$ & $\mathrm{N}$ & $\mathrm{N}$ & $\mathrm{N}$ & $\mathrm{N}$ & $\mathrm{N}$ & \\
\hline Zingiberaceae & Zingiber officinale & Zengebil & Rh. & & $\mathrm{MeOH}$ & $\mathrm{N}$ & $\mathrm{N}$ & $\mathrm{N}$ & $\mathrm{N}$ & $\mathrm{N}$ & $\mathrm{N}$ & $\mathrm{N}$ & $\mathrm{N}$ & $\mathrm{N}$ & \begin{tabular}{|l|}
$\mathrm{N}$ \\
\end{tabular} & $\mathrm{N}$ & $\mathrm{N}$ & $\mathrm{N}$ & $\mathrm{N}$ & \begin{tabular}{|l|}
$\mathrm{N}$ \\
\end{tabular} & $\mathrm{N}$ & $\mathrm{N}$ & \begin{tabular}{|l|}
$\mathrm{N}$ \\
\end{tabular} & \begin{tabular}{|l|}
$\mathrm{N}$ \\
\end{tabular} & + & Elfadel et al. \\
\hline Lingineracede & Roscoe & Lengedn & Knl. & 1014 & Hex. & $\mathrm{N}$ & $\mathrm{N}$ & $\mathrm{N}$ & $\mathrm{N}$ & $\mathrm{N}$ & $\mathrm{N}$ & $\mathrm{N}$ & $\mathrm{N}$ & $\mathrm{N}$ & $\mathrm{N}$ & $\mathrm{N}$ & $\mathrm{N}$ & $\mathrm{N}$ & $\mathrm{N}$ & $\mathrm{N}$ & $\mathrm{N}$ & $\mathrm{N}$ & \begin{tabular}{|l|}
$\mathrm{N}$ \\
\end{tabular} & \begin{tabular}{|l|l|}
$\mathrm{N}$ & \\
\end{tabular} & $\mathrm{N}$ & 2015 \\
\hline
\end{tabular}




\begin{tabular}{|c|c|c|c|c|c|c|c|c|c|c|c|c|c|c|c|c|c|c|c|c|c|c|c|c|c|}
\hline Plant Family & $\begin{array}{c}\text { Plant Scientific } \\
\text { Name }\end{array}$ & $\begin{array}{c}\text { Plant } \\
\text { Vernacular } \\
\text { Name }\end{array}$ & $\begin{array}{l}\text { Plant } \\
\text { Part }\end{array}$ & $\begin{array}{c}\text { Method } \\
\text { used }\end{array}$ & $\begin{array}{c}\text { Solvent } \\
\text { used }\end{array}$ & 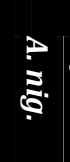 & $\stackrel{\overrightarrow{2}}{?}$ & $\stackrel{\vec{s}}{\vec{S}}$ & $\stackrel{?}{\mathfrak{s}}$ & $\vec{x}$ & $\stackrel{x}{\vdots}$ & $\frac{1}{9}$ & $\begin{array}{l}\text { Acti } \\
: \\
\overline{5}\end{array}$ & $\begin{array}{ll}\text { ivity } \\
0 \\
0 \\
0 \\
0 \\
0\end{array}$ & 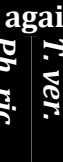 & $\begin{array}{l}\text { inst } \\
-3 \\
\vdots \\
\vdots \\
\vdots \\
-3\end{array}$ & $\mid \begin{array}{l}\text { fungi } \\
-3 \\
3 \\
0\end{array}$ & $\mid$ & $\begin{array}{l}\overrightarrow{1} \\
\hat{\mathbf{s}}\end{array}$ & $\begin{array}{l}3 \\
\hat{5}\end{array}$ & 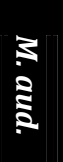 & क & $\frac{0}{n}$ & 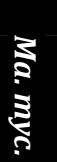 & References \\
\hline & & & & & $\begin{array}{c}\mathrm{MeOH} \\
\text { defatted }\end{array}$ & $\mathrm{N}$ & $\mathrm{N}$ & $\mathrm{N}$ & $\mathrm{N}$ & $\mathrm{N}$ & $\mathrm{N}$ & $\mathrm{N}$ & $\mathrm{N}$ & \begin{tabular}{l|l}
$\mathrm{N}$ & $\mathrm{N}$
\end{tabular} & $\mathrm{N} \mid \mathrm{N}$ & \begin{tabular}{l|l}
$N$ & $N$
\end{tabular} & $\mathrm{~J}$ & $\mathrm{~N}$ & $\mathrm{~N}$ & $\mathrm{~N}$ & $\mathrm{~N}$ & $\mathrm{~N}$ & $\mathrm{~N}$ & $\mathrm{~N}$ & \\
\hline
\end{tabular}

A. nig. $=$ Aspergillus niger,

A. fla. $=$ Aspergillus flavus,

A. fum. $=$ Aspergillus fumigatus,

C. alb. = Candida albicans,

F. oxy.= Fusarium oxysporum

F. cul. $=$ Fusarium culmorum

F. gra. $=$ Fusarium graminearum,

P. ita. $=$ Penicillium italicum

P. dig. $=$ Penicillium digitatum,

Ph. ric. $=$ Phialophora richardsiae,

T. ver. = Tricophyton verrucosum,

T. rub. = Trichophyton rubrum,

T. con. $=$ Trichophyton concentricum,

T. ton. $=$ Trichophyton tonsurans

T. men. $=$ Tricophyton mentagrophytes,

M. can. = Microsporum canis,

M. aud.= Microsporum audouinii,

S. cer. $=$ Saccharomyces cerevisiae

Ma. myc.= Madurella mycetomatis,

E. flo. $=$ Epidermophyton floccosum

di.ether= diethyl ether,

Hex. = Hexane,

Acet $=$ Acetone

Ess. Oil= Essential oil

$\mathrm{H}_{2} \mathrm{O}=$ Aqueous,

$\mathrm{MeOH}=$ Methanol,

$\mathrm{CHCl}_{3}=$ Chloroform

Pet. ether= Petroleum ether

EtOAc $=$ Ethyl acetate,

EtOH= Ethanol

Co. $=$ Corms,

A.P. $=$ Aerial parts
Tub.= Tuber,

Ro.= Roots,

Rh.= Rhizome,

Le.= Leaves,

St. $=$ Stem

W.P.= Whole Plant,

A.P.= Aerial Parts,

Fr.= Fruits,

Po.= Pods,

Se.= Seeds,

$\mathrm{Br} .=$ Branches,

Ba.= Bark,

Oleo-gum resin= O.G.R.,

Wo.P.= Woody Parts,

Me.= Mesocarp,

Pu.= Pulp,

Pe $=$ Peel

Hy.= Hypocotyls,

Ca.= Callus,

Co.= Cotyledon

Po.= Pods,

Cy.= Calyces,

$\mathrm{G} .=$ Gum.

M1=cup-plate diffusion method,

M2= Disc diffusion method

M3= Mean radial growth mycelium either after defined time or at interval periods,

M4=micro-dilution method or MIC,

$(+)=$ presence of antifungal activity

$(-)=$ absence of antifungal activity,

$\mathrm{N}=$ Not tested. 
In the current survey, the fungi that examined for their sensitivity against the selected Sudanese plants are representing 20 species belonging to 10 genera and 6 families. As shown in Table 1, the fungal genera that were sensitive to these plants were Aspergillus, Candida, Fusarium, Pencilliuum, Phialophora, Tricophyton, Saccharomyces, Madurella and Epidermophyton. Fig. 2 demonstrated that the majority of the Sudanese plants were tested against the genera Aspergillus and Candida. There is no clear reason behind why researchers gave these two genera more attention in their investigations, however, authors suggest three possible reasons: 1- these two genera are randomly selected for screening studies; 2-these two genera are easy for culturing; 3- Aspergillus was chosen as a typical fungus with much medical and commercial importance; while Candida was chosen as a typical yeast causing many superficial infections in human.

Accordingly, lots of research efforts are required to evaluate the antifungal activity of these plants against as much as possible of fungi, molds and yeasts particularly the parasitic and saprophytic ones in order to determine the antifungal properties which may lead to the discovery of new antifungal agents.

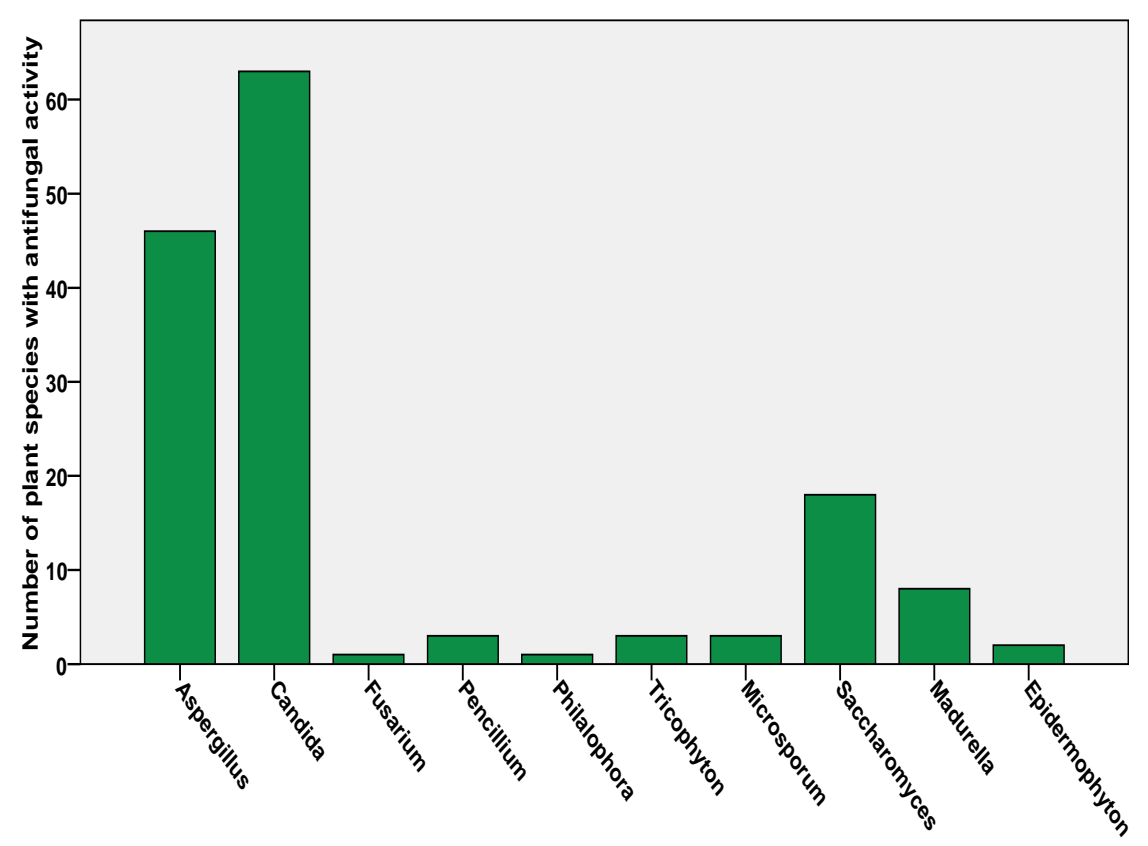

Fig. 2: The number of Sudanese plant species tested for antifungal activity compared with the screened fungal genera

\section{Conclusion}

Discovering antifungal agents from natural products remains the safer and cheaper solution for many health problems in developing countries. Systematic and more organized antifungal research is needed in Sudan to overcome the poor health situation in the country.

\section{Conflict of interest}

The authors declare that there is no conflict of interest regarding the publication of this paper.

\section{References}

Abd El Nabi OM, Reisinger EC, Reinthaler FF, Still F, Eibel U, and Krejs GJ (1992). Antimicrobial activity of Acacia nilotica (L.) Willd. ex Del. var. nilotica (Mimosaceae). Journal of Ethnopharmacology, 37: 77-79.

Abdalla AN (2004). Antimicrobial and wound healing activity of some Sudanese medicinal plants. M.Sc. Thesis, University of Khartoum.
Abdalla WE and Abdallah EM (2016). Promising Sudanese Medicinal Plants with Antibacterial Activity - a Review Article. Biological Forum, 8(1): 299-323.

Abdalla WE and Abdallah EM (2016). Promising Sudanese Medicinal Plants with Antibacterial Activity - a Review Article. Biological Forum, 8: 299-323.

Abdallah EM (2011). Plants: an alternative source for antimicrobials. Journal of Applied Pharmaceutical Science, 1(6): 16-20.

Abdallah EM, Ben Hsouna A and Al-Khalifa KS (2012). Antimicrobial, antioxidant and phytochemical investigation of Balanites aegyptiaca (L.) Del. edible fruit from Sudan. African Journal of Biotechnology, 11(52): 1153511542.

Abdel-Rahim AM, Bashiar HAM and Sulieman AA (2012a). Antimicrobial activity of the extracts of Pomegranate (Romman) Plant (Punica granatun L.). Gezira Journal of Engineering and Applied Sciences, 7(1): 1-17. 
Abdel-Rahim AM, Idris AOA and Sulieman AE (2012b). Antimicrobial activity of the extracts of Garlic and Onion. Gezira Journal of Engineering and Applied Sciences, 7(1): 1-25.

Abdel-Rahim AM, Wafa YA and Idris FA (2012c). Antimicrobial activity of the extracts of Garad (Acacia nilotica L.). Gezira Journal of Engineering and Applied Sciences, 7(2): 26-50.

Abd-Ulgadir KS, Suliman SI, Zakria IA and Hassan NA (2015). Antimicrobial potential of methanolic extracts of Hibiscus sabdariffa and Ricinus communis. Advancement in Medicinal Plant Research, 3(1): 18-22.

Ahmad I, Owais M, Shahid M and Aqil F (2010). Combating fungal infections: Problems and remedy. Springer-Verlag, Berlin Heidelberg, Germany.

Alhadi EA, Khalid HS, Alhassan MS, Kabbashi AS and Noor MO (2015). Antimicrobial and phytochemical screening of Cordia africana in Sudan. World Journal of Pharmaceutical Research, 4(3): 257-269.

Ali, E.M.M., Almagboul, A.Z.I., Khogali, S.M.E. and Gergeir, U.M.A. (2012). Antimicrobial activity of Cannabis sativa L. Chinese Medicine, 3: 61-64.

Brown AF and Massey RE (1929). Flora of the Sudan. Sudan Govt, London, UK.

Cowan MM (1999). Plant products as antimicrobial agents. Clinical Microbiology Reviews, 12(4): 564-582.

Cunningham AB (1997). An Africa-wide overview of medicinal plant harvesting, conservation and health care. Medicinal Plants for Forest Conservation and Health Care, 11: 116-129.

El Ghazali GEB, Bari EA, Bashir AK and Salih AM (1987). Medicinal plants of the Sudan, part II: Medicinal Plants of Eastern Nuba Mountains. National Council for Research, Khartoum, Sudan.

El Ghazali GEB, El Tohami MS and El Egami AAB. (1994). Medicinal Plants of the Sudan, part III: Medicinal Plants of the White Nile Province. National Centre for Research, Khartoum, Sudan.

El Ghazali GEB, El Tohami MS, El Egami AAB, Abdalla WE and Galal M (1997). Medicinal plants of the Sudan, part IV: Medicinal plants of northern Kordofan. National Centre for Research, Khartoum, Sudan.

Elegami AA, Elnima EI, Muddathir AK and Omer ME (2001). Antimicrobial activity of Plicosepalus acaciae. Fitoterapia, 72: 431-434.

El-Egami AA, El-Tohami MS, El-Nima EI and Muddathir AK (2005). In vitro antimicrobial activities of Nymphaea lotus and Jussiaea erecta. Omdurman Journal of Pharmaceutical Sciences, 1(1): 117-123.
ElNour MEM, Ali AMA and Saeed BAE (2015). Antimicrobial activities and phytochemical screening of Callus and seeds extracts of Fenugreek (Trigonella foenum-graecum). International Journal of Current Microbiology and Applied Sciences, 4(2): 147-157.

ElNour MEM, Mahmood FZA and Yagoub SO (2014). Callus induction and antimicrobial activities of callus and intact plant extracts of Datura stramonium L. International Journal of Science and Research, 3(8): 1105-1109.

Hassan LEA, Sirat HM, Yagi SMA, Koko WS and Abdelwahab SI (2011). In vitro antimicrobial activities of chloroformic, hexane and ethanolic extracts of Citrullus lanatus var. citroides (Wild melon). Journal of Medicinal Plant Research, 5(8): 1338-1344.

Kabbashi AS, Garbi MI, Osman EA, Dahab MM, Koko WS, Abuzeid N, A Salam HA and Ahmed IF (2015a). In vitro antimicrobial activity and cytotoxicity of ethanolic leaves extract of Acacia nilotica. International Journal of Multidisciplinary Research and Development, 2(10): 172-176.

Kabbashi AS, Garbi MI, Osman EE, Dahab MM, Koko WS and Abuzeid N (2015b). In vitro antimicrobial activity of ethanolic seeds extract of Nigella sativa (Linn.) in Sudan. African Journal of Microbiology Research, 9(11): 788-792.

Kabbashi AS, Koko WS, Mohammed SA, Musa N, Osman EE, Dahab MM, Fadul Allah EF and Mohammed AK (2014). In vitro amoebicidal, antimicrobial and antioxidant activities of the plants Adansonia digitata and Cucurbita maxima. Advancement in Medicinal Plant Research, 2(3): 50-57.

Kabbashi AS, Mohammed SEA, Almagboul AZ and Ahmed IF (2015c). Antimicrobial activity and cytotoxicity of ethanolic extract of Cyperus rotundus L. American Journal of Pharmacy and Pharmaceutical Sciences, 2(1): 1-13.

Khalid H, Abdalla WE, Abdelgadir H, Opatz T and Efferth T (2012). Gems from traditional northAfrican medicine: medicinal and aromatic plants from Sudan. Natural products and Bioprospecting, 2(3): 92-103.

Moglad EHO, Abdalla OM, Abd Algadir H, Koko WS. and Saadabi AM (2014). In vitro antimicrobial activity and cytotoxicity of Maerua oblongifolia. International Journal of Medicine and Medical Sciences, 1(3): 32-37.

Moglad EHO, Alhassan MS, Koko WS and Saadabi AM (2012). In vitro antimicrobial activity of Sudanese medicinal plants. Journal of Medical Sciences, 12(7): 219-223.

Mosa EO, Justin DD, Hamam SB, Omer MEA and Ayoub SMH (2014). Evaluation of phytochemical and antimicrobial activities of some Sudanese 
medicinal plants. World Journal of Pharmacy and Pharmaceutical Sciences, 3(12): 1769-1776.

Musa AE and Gasmelseed GA (2014). Application of Acacia nilotica spp nilotica Pods (Garad) powder as alternative vegetable retanning material. Journal of Forest Products and Industries, 3(2): 112-117.

Omer MEFA and Elnima EI (2003). Antimicrobial activity of Ximenia americana. Fitoterapia, 74: 122-126.

Omer MYA (2007). Survey and identification of fungal diseases of import plant crops in the White Nile state. M.Sc. thesis, University of Khartoum.

Razzaghi-Abyaneh M and Rai M (2013). Antifungal metabolites from plants. Springer-Verlag, Berlin Heidelberg, Germany.

Rodino S, Butu M, Petrache P, Butu A, and Cornea C P (2014). Antifungal activity of four plants against Alternaria alternata. Scientific Bulletin. Series F. Biotechnologies, 18: 60-65.

Saadabi AM and Abu Zaid IE (2011). An in vitro antimicrobial activity of Moringa oleifera L. seed extracts against different groups of microorganisms. Australian Journal of Basic and Applied Sciences, 5(5): 129-134.

Saadabi AMA (2007). Evaluation of Lawsonia inermis Linn. (Sudanese Henna) leaf extracts as an antimicrobial agent. Research Journal of Biological Sciences, 2(4): 419-423.
Saadabi AMA and Ayoub SMH (2009). Comparative bioactivity of Hydnora abyssinica A. Braun against different groups of fungi and bacteria. Journal of Medicinal Plants Research, 3(4): 262-265.

Sasidharan S, Ping KY, Latha LY and Lachumy SJ (2012). Screening methods in the study of fungicidal property of medicinal plants. INTECH Open Access Publisher. In: Dhanasekaran D, Thajuddin N and Panneerselvam A (eds.), Fungicides for plant and animal diseases. Tech publisher. Riheka, Croatia: 107-118.

Scorzoni L, Sangalli-Leite F, de Lacorte Singulani J, Costa-Orlandi CB, Fusco-Almeida AM and Mendes-Giannini MJS (2016). Searching new antifungals: The use of in vitro and in vivo methods for evaluation of natural compounds. Journal of Microbiological Methods, 123: 68-78.

Sirag N, Yassin S and Yousif MA (2009). Antimicrobial activity of three medicinal plants. Gezira Journal of Health Sciences, 5(2): 56-61.

Sulieman AME, Ahmed HE and Abdelrahim AM (2008). The chemical composition of Fenugreek (Trigonella foenum-graceum L.) and the antimicrobial properties of its seed oil. Gezira Journal of Engineering and Applied Sciences, $3(2): 1-21$.

Yang CR, Zhang Y, Jacob MR, Khan SI, Zhang YJ and Li XC (2006). Antifungal activity of C-27 steroidal saponins. Antimicrobial Agents and Chemotherapy, 50(5): 1710-1714. 